\title{
Adaptive Control of Aircraft in Uncertain Icing Conditions
}

\author{
A Thesis \\ Presented to \\ the Faculty of the School of Engineering and Applied Science \\ UNIVERSITY OF VIRGINIA
}

In Partial Fulfillment

of the Requirements for the Degree

Master of Science in Electrical Engineering

by

Korey MatThew RANKin

May 2014 


\section{APPROVAL SHEET}

The thesis

is submitted in partial fulfillment of the requirements

for the degree of

Master of Science

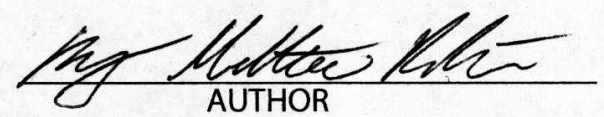

The thesis has been read and approved by the examining committee:

\begin{tabular}{c} 
Prof. Gang Tao \\
\hline $\begin{array}{c}\text { Advisor } \\
\text { Prof. Zongli Lin }\end{array}$ \\
\hline Prof. Scott Acton \\
\hline
\end{tabular}

Accepted for the School of Engineering and Applied Science:

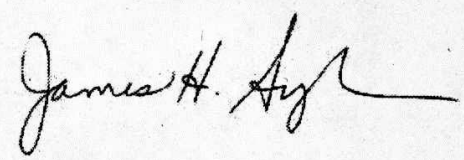

Dean, School of Engineering and Applied Science

May

2014 


\section{Acknowledgements}

I would like to extend my gratitude to Dr. Philip Spickler and Dr. Richard Bowman. If it were not for their insistence I would never have believed I could obtain a graduate degree.

I would also like to thank Dr. Gang Tao for his guidance and encouragement. I have found inspiration in his achievements and the knowledge he has shared with me. He has challenged me continuously, all the meanwhile providing me with the confidence that every task would be overcome. I consider it a great priviledge to have studied under him the past two years.

Finally, I would like to thank my parent's, Dale and Lisa, for their support and faith in me. I could not have pursued a higher education if it were not for them. I owe all that I have to them. 


\section{Abstract}

Ice accretion on aircraft is, at times, unavoidable. The unpredictable effects of ice on aircraft dynamics makes an encounter with a weather pattern where ice accretion is likely a dire situation. The many shapes and areas on the aircraft where ice can form can either have no effect on the control or total loss of control of the aircraft. The objective of this research is to first present the results of a modeling study of the effects of ice on the equations of motion of an airplane. The next objective is to show that an adaptive control scheme can compensate for the unpredictable and detrimental effects ice accretion has on aircraft stability and control. A linearized model of a Cessna 208 Super Cargomaster is presented for situations where the aircraft is in no icing conditions, fully iced, and horizontal tail icing only. The latter two icing conditions are to be the worst case scenarios of such a situation. The iced aircraft models are based on data taken from the effects of ice accretion on a DeHavilland Twin Otter aircraft. Simulations for a classical fixed gain controller as well as an adaptive output tracking scheme are presented for comparison purposes. The classical fixed gain control simulations illustrate the detrimental effects of ice accretion on flight control while the adaptive output tracking controller simulations

detail the effectiveness of adaptive control to handle the resultant changes in aircraft dynamics. 


\section{Contents}

1 Introduction 1

1.1 Research Motivation ................... 1

1.2 Literature Review . . . . . . . . . . . . . . . . . . . 5

1.3 Thesis Outline . . . . . . . . . . . . . . . . . . . . 7

2 Background $\quad 8$

2.1 Control System Models . . . . . . . . . . . . . . . . . 8

2.2 System Stability . . . . . . . . . . . . . . . . . . 11

2.3 Classical Control . . . . . . . . . . . . . . . . 16

2.4 Adaptive Control . . . . . . . . . . . . . . . . . . . . . 16

3 Aircraft Models with Icing $\quad 18$

3.1 Physics of Icing . . . . . . . . . . . . . . . . . . . . . 19

3.2 Aircraft Models without Icing . . . . . . . . . . . . . . . . . 24

3.2.1 Equations of Motion without Icing . . . . . . . . . . . 25

3.2.2 State Space Models without Icing . . . . . . . . . . . . . . . . 30

3.3 Aircraft Models with Icing . . . . . . . . . . . . . . . . 36 
3.3 .1 Modeling Ice Accretion . . . . . . . . . . . . . . . . . . . 36

3.3.2 Equations of Motion with Icing . . . . . . . . . . . . . 45

3.3.3 State Space Models with Icing . . . . . . . . . . . . . . . . 48

3.4 Summary .............................. 55

4 Adaptive Control Design $\quad 56$

4.1 Control Objectives and Design Conditions . . . . . . . . . . . . 56

4.1.1 Limitations of State Tracking Design . . . . . . . . . . . . 57

4.1.2 Matching Conditions for Output Tracking . . . . . . . . . . . 59

4.2 Output Tracking Control Design . . . . . . . . . . . . . . 61

4.2.1 Design Condition Verification ............. 61

4.2 .2 Controller Structure . . . . . . . . . . . . . . . 69

4.2 .3 Estimation Error . . . . . . . . . . . . . . 69

4.2.4 Adaptive Laws . . . . . . . . . . . . . . . . . . 71

4.3 Stability Properties . . . . . . . . . . . . . . . . 72

4.4 Summary . . . . . . . . . . . . . . . . . 73

5 Adaptive Control System Simulations $\quad 75$

5.1 Worst Case Scenario Icing . . . . . . . . . . . . . . . . . 76

5.1.1 Longitudinal Dynamic Model . . . . . . . . . . . . 76

5.1 .2 Lateral-Directional Dynamic Model . . . . . . . . . . . . 87

5.2 Worst Case Scenario Horizontal Tail Icing . . . . . . . . . . . . . . . 94

5.2.1 Longitudinal Dynamic Model . . . . . . . . . . . . . . . . . . 94

5.2.2 Lateral-Directional Dynamic Model . . . . . . . . . . . . 103 
5.3 Summary . . . . . . . . . . . . . . . . . . . 110

6 Conclusions and Future Work 111

6.1 Summary and Conclusions . . . . . . . . . . . . . . . . 111

6.2 Future Research Topics . . . . . . . . . . . . . . . . . . . . . . . . . 112

$\begin{array}{ll}\text { References } & 115\end{array}$ 


\section{List of Figures}

2.1 Block diagram of a system with output feedback and an adjustable preamplifier gain $k \ldots \ldots \ldots \ldots \ldots \ldots \ldots \ldots$

2.2 State space representation of a plant with state feedback. . . . . . 17

3.1 Body, wind, and stability axis systems and aerodynamic angles. Re-

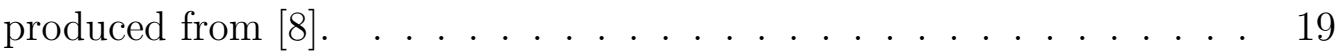

3.2 Severe mixed ice accumulation on the tail of a full-scale NASA test aircraft. Reproduced from $[56] . \ldots \ldots \ldots$. . . . . . . . 21

3.3 Upper surface separation bubble aft of leading-edge ice accretion. Reproduced from $[22] \ldots \ldots \ldots \ldots \ldots \ldots \ldots$

5.1 Output tracking error for the fixed gain control of the angle of attack, $\alpha$, in a worst case scenario icing encounter at $t=30 \mathrm{~s} . \ldots \ldots 79$

5.2 Output tracking error for the adaptive control of the angle of attack, $\alpha$, in a worst case scenario icing encounter at $t=30 \mathrm{~s} \ldots \ldots . \ldots 79$

5.3 Parameter errors for $\theta_{1}, \theta_{2}$, and $\theta_{3}$ for the adaptive control of the angle of attack, $\alpha$, in a worst case scenario icing encounter at $t=30 \mathrm{~s} . \ldots 80$ 
5.4 Parameter errors for $\theta_{4}, \theta_{5}$, and $\rho$ for the adaptive control of the angle of attack, $\alpha$, in a worst case scenario icing encounter at $t=30 \mathrm{~s} . \ldots 81$

5.5 Output tracking error for the fixed gain control of the pitch attitude angle, $\theta$, in a worst case scenario icing encounter at $t=30 \mathrm{~s} . \quad . \quad . \quad 82$

5.6 Output tracking error for the adaptive control of the pitch attitude angle, $\theta$, in a worst case scenario icing encounter at $t=30 \mathrm{~s} . \quad . \quad . \quad 82$

5.7 Parameter errors for $\theta_{1}, \theta_{2}$, and $\theta_{3}$ for the adaptive control of the pitch attitude angle, $\theta$, in a worst case scenario icing encounter at $t=30 \mathrm{~s}$.

5.8 Parameter errors for $\theta_{4}, \theta_{5}$, and $\rho$ for the adaptive control of the pitch attitude angle, $\theta$, in a worst case scenario icing encounter at $t=30 \mathrm{~s} . \quad 84$

5.9 Output tracking error for the fixed gain control of the heading angle, $\psi$, in a worst case scenario icing encounter at $t=30 \mathrm{~s} . . . . . \quad$. 89

5.10 Output tracking error for the adaptive control of the heading angle, $\psi$, in a worst case scenario icing encounter at $t=30 \mathrm{~s} . \ldots . .$.

5.11 Parameter errors for $\theta_{1}, \theta_{2}$, and $\theta_{3}$ for the adaptive control of the heading angle, $\psi$, in a worst case scenario icing encounter at $t=30 \mathrm{~s} . \quad 90$

5.12 Parameter errors for $\theta_{4}$ and $\theta_{5}$ for the adaptive control of the heading angle, $\psi$, in a worst case scenario icing encounter at $t=30 \mathrm{~s} . . . .991$

5.13 Parameter errors for $\theta_{6}$ and $\rho$ for the adaptive control of the heading angle, $\psi$, in a worst case scenario icing encounter at $t=30 \mathrm{~s} . . . . \quad 92$

5.14 Output tracking error for the fixed gain control of the angle of attack, $\alpha$, in a horizontal tail worst case scenario icing encounter at $t=30 \mathrm{~s}$. 
5.15 Output tracking error for the adaptive control of the angle of attack, $\alpha$, in a horizontal tail worst case scenario icing encounter at $t=30 \mathrm{~s}$.

5.16 Parameter errors for $\theta_{1}, \theta_{2}$, and $\theta_{3}$ for the adaptive control of the angle of attack, $\alpha$, in a horizontal tail worst case scenario icing encounter at $t=30 \mathrm{~s} \ldots \ldots \ldots \ldots \ldots \ldots$

5.17 Parameter errors for $\theta_{4}, \theta_{5}$, and $\rho$ for the adaptive control of the angle of attack, $\alpha$, in a horizontal tail worst case scenario icing encounter at $t=30 \mathrm{~s}$

5.18 Output tracking error for the fixed gain control of the pitch attitude angle, $\theta$, in a horizontal tail worst case scenario icing encounter at $t=30 \mathrm{~s} \ldots \ldots \ldots \ldots \ldots \ldots \ldots$

5.19 Output tracking error for the adaptive control of the pitch attitude angle, $\theta$, in a horizontal tail worst case scenario icing encounter at $t=30 \mathrm{~s}$

5.20 Parameter errors for $\theta_{1}, \theta_{2}$, and $\theta_{3}$ for the adaptive control of the pitch attitude angle, $\theta$, in a horizontal tail worst case scenario icing encounter at $t=30 \mathrm{~s} \ldots \ldots \ldots$. . . . . . . . . . . 100

5.21 Parameter errors for $\theta_{4}, \theta_{5}$, and $\rho$ for the adaptive control of the pitch attitude angle, $\theta$, in a horizontal tail worst case scenario icing encounter at $t=30 \mathrm{~s} \ldots \ldots \ldots \ldots 10 \ldots \ldots$

5.22 Output tracking error for the fixed gain control of the heading angle, $\psi$, in a horizontal tail worst case scenario icing encounter at $t=30 \mathrm{~s} . \quad 105$ 
5.23 Output tracking error for the adaptive control of the heading angle, $\psi$, in a horizontal tail worst case scenario icing encounter at $t=30 \mathrm{~s} . \quad 105$

5.24 Parameter errors for $\theta_{1}, \theta_{2}$, and $\theta_{3}$ for the adaptive control of the heading angle, $\psi$, in a horizontal tail worst case scenario icing encounter at $t=30 \mathrm{~s} \ldots \ldots \ldots \ldots$. . . . . . . . . . . . . . 106

5.25 Parameter errors for $\theta_{4}$ and $\theta_{5}$ for the adaptive control of the heading angle, $\psi$, in a horizontal tail worst case scenario icing encounter at

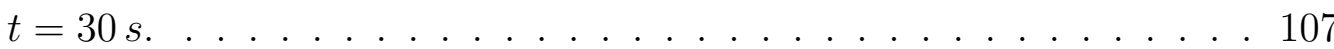

5.26 Parameter errors for $\theta_{6}$ and $\rho$ for the adaptive control of the heading angle, $\psi$, in a horizontal tail worst case scenario icing encounter at

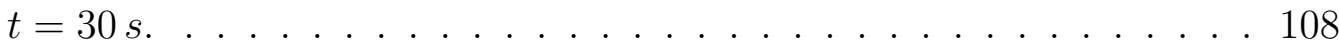




\section{List of Tables}

3.1 Change in stability and control parameters $\left(f_{\text {ice }}\right)$ due to icing [3] [4]. . 38

4.1 Transfer functions for the worst case scenario icing longitudinal state space model. . . . . . . . . . . . . . . . . . 63

4.2 Transfer functions for the worst case scenario horizontal tail icing longitudinal state space model. . . . . . . . . . . . . . . . . . . . . . . 64

4.3 Transfer functions for the worst case scenario icing lateral-directional state space model. . . . . . . . . . . . . . . . . . . . . . . 66

4.4 Transfer functions for the worst case scenario horizontal tail icing lateral-directional state space model. . . . . . . . . . . . . . . 68 


\section{Nomenclature}

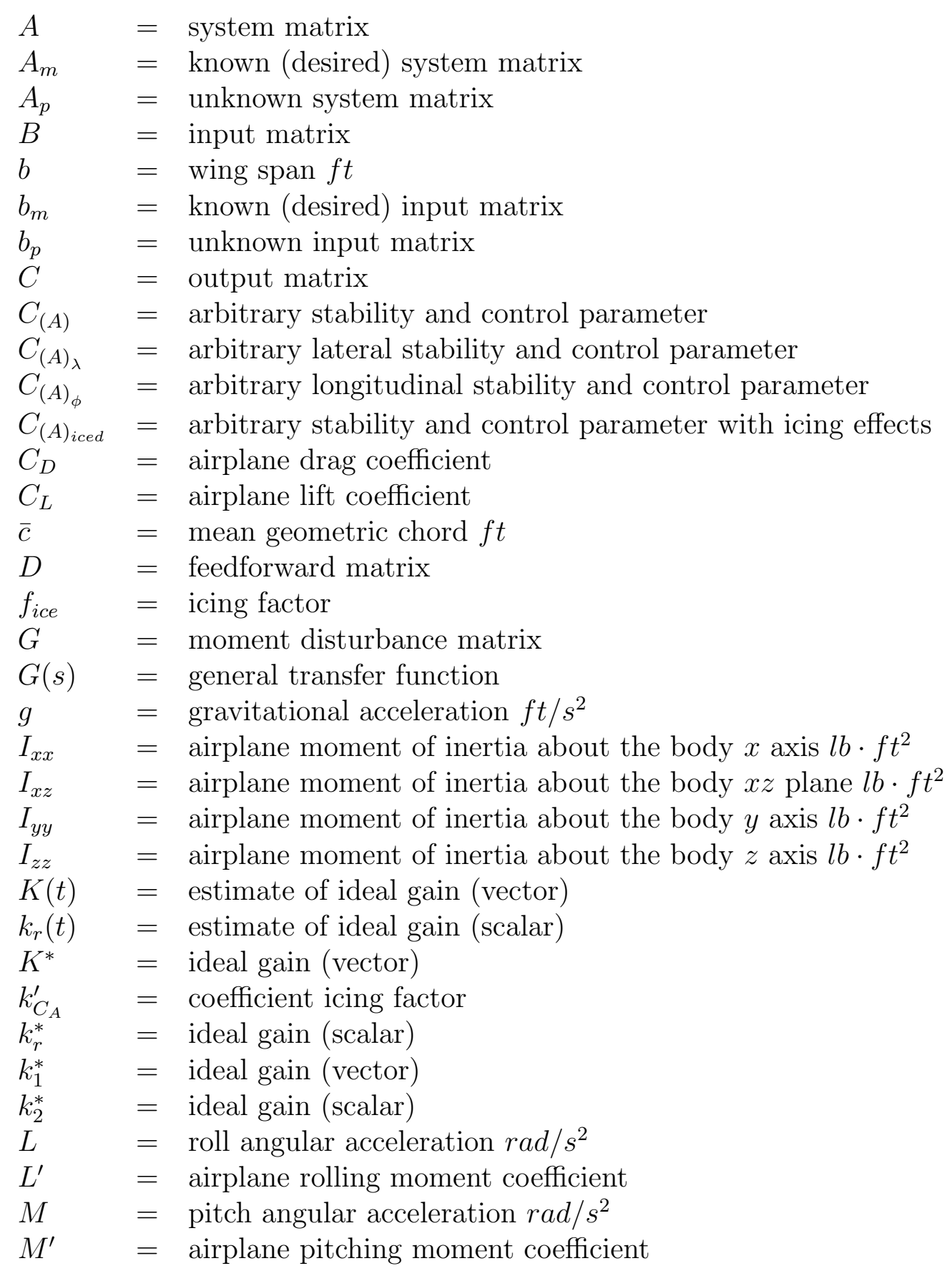




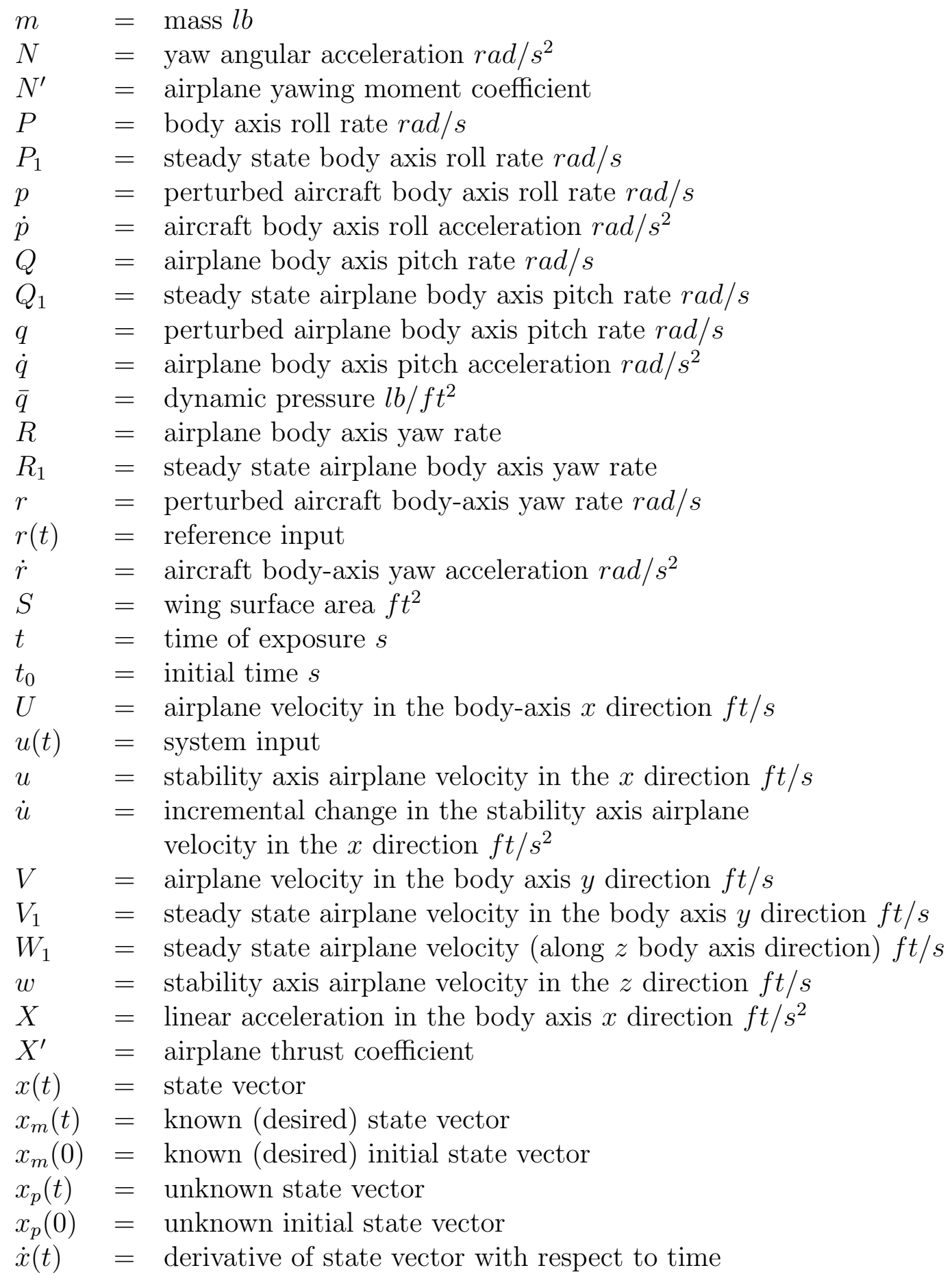


$\dot{x}(t)=$ derivative of known (desired) state vector with respect to time

$\dot{x}_{p}(t)=$ derivative of unknown state vector with respect to time

$Y=$ linear acceleration in the body axis $y$ direction $\mathrm{ft} / \mathrm{s}^{2}$

$Y^{\prime}=$ airplane side-force coefficient

$y(t)=$ system output

$y_{m}(t)=$ reference output

$Z=$ linear acceleration in the body axis $z$ direction $\mathrm{ft} / \mathrm{s}^{2}$

$Z^{\prime} \quad=$ airplane lift coefficient

\section{Greek Symbols}

$\alpha=$ angle of attack rad

$\dot{\alpha}=$ incremental change in angle of attack $\mathrm{rad} / \mathrm{s}$

$\beta=$ sideslip angle rad

$\dot{\beta}=$ incremental change in sideslip angle $\mathrm{rad} / \mathrm{s}$

$\Delta=$ change

$\delta=$ control deflection angle rad

$\eta_{\text {ice }}=$ icing severity parameter

$\Theta=$ aircraft pitch attitude angle $\mathrm{rad}$

$\Theta_{1}=$ steady-state aircraft pitch attitude angle rad

$\theta=$ pitch attitude angle $\mathrm{rad}$

$\dot{\theta}=$ incremental change in pitch attitude angle $\mathrm{rad} / \mathrm{s}$

$\Phi=$ airplane roll attitude angle rad

$\Phi_{1}=$ steady state airplane roll attitude angle $\mathrm{rad}$

$\phi \quad=$ roll attitude angle $\mathrm{rad}$

$\dot{\phi}=$ incremental change in roll attitude angle $\mathrm{rad} / \mathrm{s}$

$\psi \quad=$ heading angle rad

$\dot{\psi}=$ incremental change in heading angle $\mathrm{rad} / \mathrm{s}$

$\dot{\omega}=$ incremental change in the stability axis aircraft velocity in the $z$ direction $\mathrm{ft} / \mathrm{s}^{2}$ 


\section{Subscripts}

$0=$ derivative with respect to zero angle of attack: initial condition

$1=$ steady state; first mode

$a=$ aileron

$D \quad=$ drag

e $=$ elevator

$H=$ horizontal tail

ice $=$ ice accretion effect

iced $=$ ice accretion effect

$L=$ lift

$L W=$ left wing

$p=$ derivative with respect to aircraft roll rate

$q=$ derivative with respect to airplane pitch rate

$R=$ rudder

$R W=$ right wing

$r=$ derivative with respect to aircraft yaw rate

$T=$ thrust

$T_{\alpha}=$ derivative with respect to change in angle of attack due to thrust

$T_{u}=$ derivative with respect to change in speed due to thrust

$u=$ derivative with respect to stability $\mathrm{x}$-axis airplane velocity

$W=$ wings

$W F=$ wings and fuselage

$\alpha=$ derivative with respect to angle of attack

$\dot{\alpha}=$ derivative with respect to incremental change in angle of attack

$\beta=$ derivative with respect to sideslip angle

$\delta_{a}=$ derivative with respect to aileron deflection angle

$\delta_{e}=$ derivative with respect to elevator deflection angle

$\delta_{r}=$ derivative with respect to rudder deflection angle

$\delta_{T}=$ derivative with respect to thrust

$\theta=$ derivative with respect to pitch attitude angle

$\lambda=$ with respect to lateral-directional stability

$\phi \quad=$ with respect to longitudinal stability

$\%=$ percentage 


\section{Chapter 1}

\section{Introduction}

\section{$1.1 \quad$ Research Motivation}

Icing has been a major concern for aviation for many years. It has been recorded by the Air Safety Foundation that from the year 1990 to 2000, 3,230 aircraft accidents occured with $12 \%$ of that number being related to icing [7]. According to [56], experienced pilots have between five to eight minutes, or perhaps even less, to escape harmful icing conditions before the aircraft will experience a degrade in performance. The trouble with this is that it can be hard for a pilot to realize he has flown into

such harmful conditions. More harrowing still is that while in cruise the effects of ice accretion might be unobserved, meaning they are having no effect on the planes performance whatsoever until the plane is commanded away from this steady state.

American Eagle flight 4184 is a specific example of the catastrophic effects icing can have on the control of an aircraft. The aircraft in question had been directed 
into a holding pattern. During this time, the airplane encountered an intermittent pattern of supercooled cloud and drizzle/rain drops. The size of these drops were greater than those that the aircarft was able to maintain flight in per the icing certification envelope. A ridge of ice formed aft of the deicing boots which in turn led to a hinge moment reversal. The resulting uncommanded roll caused the autopilot to automatically shutoff and a departure from controlled flight to occur, which the pilots were incapable of overcoming. All 68 onboard, including the captain, first officer, two flight attendants, and 64 passengers, sustained fatal injuries [40].

Global Aviation Glo-Air flight 73 is another specific example of an accident due to icing. The aircraft in question had been awaiting takeoff in potential icing conditions. Though freezing rain was not evident, it was snowing in the, approximately, 40 minutes that expired prior to takeoff. Ground crew members had noticed ice on the wings but the extent to which it had accumulated was not clear. The first officer had noticed the icing as well but felt that it would be negligible to flight operations. Upon takeoff, the aircraft only gained about 20 to 50 feet in altitude before an uncommanded roll, caused by ice accretion, coupled with the resultant actions by the captain to regain control of the aircraft caused the left wingtip to collide with the ground followed by the fuselage. Approximately 8.7 seconds expired between liftoff and the resulting crash [41]. It is stated in [41] that "of the six occupants on board, the captain, the flight attendant, and one passenger were killed, and the first officer and two passengers were seriously injured. The airplane was destroyed by impact forces and postcrash fire."

Another more recent example of an accident due to icing is that of a Cessna 
Citation 560, N500AT. The aircraft was operated by Martinair, Inc., for Circuit City Stores, Inc. While descending during normal landing procedures, the aircraft encountered icing conditions. The "sister ship" following closely behind the accident aircraft observed no visible precipitation but did notice the accumulation of rime ice on the aircraft. There is no record on the cockpit voice recording that the flight crew of the accident airplane noticed any precipitation, though they did observe a buildup of ice on their aircraft as well. The "sister ship" was able to land safely by utilizing the deicing mechanisms of the plane often. The accident aircraft was using the deicing mechanisms to its advantage as well, but not as often since no adverse effects to the behaviour of the aircraft had been noticed.

As the accident airplane was descending to approximately 6,000 ft the airplane experienced an uncommanded roll to the left along with a decrease in pitch. According to [43], "the autopilot . . . can be automatically disconnected if certain conditions occur, such as a roll angle of more than $40^{\circ}$ or a roll rate of more than $20^{\circ}$ per second." The uncommanded roll encountered by the accident aircraft was large enough to warrant an automatic disconnect of the autopilot leaving the flight crew to regain control of the aircraft manually. Post autopilot disconnect, the accident aircraft plummeted approximately 1,178 ft in about 15 seconds. Shortly thereafter the accident aircraft collided with the ground. The two pilots and six passengers onboard were killed. The accident aircraft was destroyed by the impact and postcrash fire. The probable cause of the accident was determined by [43] to be an aerodynamic stall resulting from ice accretion.

The aircraft that will be analyzed in this thesis is the small Cessna 208 Super 
Cargomaster. This particular aircraft has become infamous for accidents involving ice accretion. Between 1987 and 2003, there were 26 icing related incidents for the Cessna 208 Super Cargomaster [14]. The National Transportation Safety Board has listed icing as the probable cause for a multitude of Cessna 208 accidents since 2003. Links to accident reports for these incidents can be found in [39]. One major cause for these accidents is tail stall due to ice accretion. It is not reported often that this is the probable cause of an accident, however, the nature of an accident suggests whether or not it is the case. A common theme in accidents caused by horizontal tail icing is an uncommanded downward pitch. For other behaviors that are related to tail stall see [17].

As an example of an accident involving a Cessna 208 Super Cargomaster, consider the Cessna 208B, aircraft registration number N208WE, that crashed outside of Oak Glen, CA, on March 28, 2006. The aircraft was amidst a climb in altitude to 8, $800 \mathrm{ft}$ when it attempted to conduct a maneuver away from its current steady state. There is no data available from the aircraft other than radar information to determine when the departure from controlled flight began. However, eyewitnesses on the ground recalled seeing the plane approaching the ground "almost straight down". The eyewitnesses described the weather as "cold and drizzling rain, with reduced visibility due to the clouds". Shortly after the accident took place the eyewitnesses recalled that it began to sleet and snow. Both the private and commerical pilots onboard the aircraft perished [42].

Due to accidents as those cited above, the FAA recommends that the autopilot be disengaged during flight in known icing conditions. However, there are aircraft 
where the autopilot is allowed to remain activated due to the aircraft meeting FAA icing certification requirements. Another, more pressing, problem is when a flight crew is unaware that they have entered icing conditions. Aircraft response due to commanded inputs becomes a critical safety issue due to the situation when the iced airplane is commanded as a noniced airplane would be. An input that would otherwise be normal for a noniced airplane could be overly aggressive for the iced airplane. This could produce excessive climb rates leading to stall, overshoots and/or undershoots in commanded altitude.

Accidents such as those discussed above could be made largely avoidable with the development of an adaptive control system. The changes in aerodynamics warranted by the disturbance in airflow over the surfaces of an aircraft due to icing could be counteracted as the adaptive controller is updating its parameters online. Apart from any developments in de-icing equipment, there is no other technology available that is more suited to compensating for the, at times, radical changes in behaviour of an aircraft in icing conditions.

\subsection{Literature Review}

There has been little contribution to solving the problem of the control of aircraft in icing conditions. This is due, in part, to the difficulty to model an iced aircraft. Most of the research being performed currently deals with the modeling of where and how the ice accretion will form. Due to the modest understanding of this phenomena, little attention has been devoted to the resultant aerodynamic effects. Despite this, 
there has been research into developing models that are useful for control design.

The work detailed in [34], [33], [32], and [12] explains the progress being made to create smart icing system technology. As a by-product of this effort a simple method of altering stability and control parameters effected by icing has been developed. This method allows for changes in the aircraft aerodynamics due to ice accretion to be represented mathematically. This research, as it pertains to aircraft dynamics, was extended in [4], [3], and [5]. The body of work mentioned in the previous references form the basis for the effort presented in this thesis.

A slightly different approach to modeling ice accretion on aircraft, compared to the method presented in [4], [3], and [5], is presented in [54]. From data obtained in wind tunnel tests, the force and moment coefficients were broken down into components. Each component's value could be found from data tables constructed from the information learned from the wind tunnel tests. The values for each component vary depending on the data set corresponding to an icing condition.

Other research focused primarily on the prediction of ice accretion and its effects can be found in [19], [30], [52], [53], [47], [57] and [56]. There has also been research, like that presented in [6] and [55], on the detection of atmospheric icing conditions. This aforementioned work, though informative on the physical aspects of ice accretion, lends little to an approach for control analysis.

The past and current research into the control of aircraft in icing conditions is limited to that which is described in [44]. It is currently being applied to develop a controller that will handle situations in which components of the aircraft fail or the flight dynamics change considerably. An overview of the theory and related 
applications is detailed in [24].

\subsection{Thesis Outline}

This thesis is organized as follows. Chapter 2 describes the necessary background information needed to discuss the adaptive output tracking algorithm used. Information pertaining to control system models, system stability, classical control, and adaptive control will be presented. In chapter 3, the aircraft models with and without icing will be given. The physical effects of icing as well as the procedure for making changes to the models to account for ice accretion will be presented as well. Chapter 4 details the control objectives, design conditions, and design procedure for output tracking. Stability properties of the chosen output tracking method are discussed. Chapter 5 presents simulations for both a classical control law and the stated adaptive control law for worst case scenario and worst case scenario horizontal tail icing conditions. Finally, chapter 6 discusses the results presented in this thesis and the potential for future work. 


\section{Chapter 2}

\section{Background}

Before discussing the adaptive control of aircraft in uncertain icing conditions it is necessary to present some basic background information. The topics to be discussed are the development of a control system model, system stability, classical control, and adaptive control.

\subsection{Control System Models}

Control system models are derived from the differential equations that describe the input/output behaviour of a system. For this work the nonlinear dynamics of a system will be ignored, though this is not always desireable depending on the system to be analyzed. However, with a general $n^{\text {th }}$-order linear time invariant differential equation such as

$$
\frac{d^{m} y(t)}{d t^{n}}+a_{n-1} \frac{d^{m-1} y(t)}{d t^{n-1}}+\cdots+a_{0} y(t)=b_{m} \frac{d^{m} u(t)}{d t^{m}}+b_{m-1} \frac{d^{m-1} u(t)}{d t^{m-1}}+\cdots+b_{0} u(t)
$$


where $a_{i}$ and $b_{i}$ are the system parameters, it can be difficult to quickly analyze such behaviour. This has lead to two approaches to developing control system models. The first being what has become known as the classical approach and the second being the so-called modern approach [45].

\section{Classical Approach to Modeling}

The classical approach to obtaining a model of a system is to first find the differential equations describing the system. Once the equations have been obtained the laplace transform is used to turn the $n^{\text {th }}$-order differential equations dependent on the time, $t$, into an $n^{\text {th }}$-order algebraic equation dependent on the complex variable, $s$. From this point it is easy to manipulate the equation to obtain an input/output description of the system, referred to as a transfer function [45].

\section{Modern Approach to Modeling}

The modern approach to modeling a system is more commonly referred to as the state-space approach. The state-space model of a system overcomes some of the limitations of the transfer function. The most important distinctions between the two are that nonlinear, time-varying, and multiple-input multiple-output systems can be represented with a state-space model.

In general, there are four steps that need to be taken in order to derive a statespace model.

Step 1 Choose the state variables. These must be picked so that the minimum number of state variables chosen describes the state of the system completely. More 
importantly, the state variables must be linearly independent of one another.

Step 2 Find $n$ simultaneous first order differential equations in terms of the state variables. These equations are known as the state equations.

Step 3 This step depends on whether knowledge of the input for $t \geq t_{0}$ and the initial condition of each state variable at an intial time, $t_{0}$, is available. If this information is available then the state equations can be solved for the state variables.

Step 4 Algebraically combine the state variables with the system's input to find all other system variables for $t \geq t_{0}$. These equations are known as the output equations.

The state equatons and output equations found in steps 2 and 4 are known collectively as the state-space model of a system [45]. For more information on how to obtain the state-space model as it relates to aircraft please see references [16], [18], [36], and $[26]$.

With the process of how to select the state variables and derive the state and output equations given, it is important to define how a state-space model is commonly represented. The derivative of the state variables with respect to time are taken and placed in a vector, $\dot{x}(t) \in \mathbb{R}^{n \times 1}$. The state variables are taken and placed into a vector, $x(t) \in \mathbb{R}^{n \times 1}$, called the state vector. The coefficients relative to a state variable for each differential equation are taken and placed in a matrix, $A \in \mathbb{R}^{n \times n}$, called the system matrix. The coefficients relative to the input for each differential equation are placed in a matrix, $B \in \mathbb{R}^{n \times m}$, called the input matrix. The input is 
denoted as $u(t) \in \mathbb{R}^{m \times 1}$. The output is denoted as $y(t) \in \mathbb{R}^{r \times 1}$. The coefficients relative to any of the unsolved for variables in the output equation are taken and placed in a matrix, $C \in \mathbb{R}^{r \times n}$, called the output matrix. For some systems there is a matrix, $D \in \mathbb{R}^{r \times m}$, called the feedforward matrix that contains any feedforward terms in the system. For this research the feedforward matrix will consist entirely of zeros since there are no feedforward terms multiplied by the input. Altogether, the previously described vectors and matrices form the state space representation defined as

$$
\dot{x}(t)=A x(t)+B u(t), y(t)=C x(t)+D u(t)
$$

\subsection{System Stability}

The concept of stability is crucial to control system design. An unstable control system is useless and dangerous. The methods available to examine the poles depend on the representation of the system model. If the classical approach is taken then the poles of the transfer function can be examined. If the modern approach is used then the eigenvalues, which are the poles, of the system matrix $A$ can be analyzed. Either approach can quickly give information on whether or not the system is inherently stable, marginally stable, or unstable.

For adaptive control systems stability must be defined another way since knowledge of the system parameters are unavailable and possibly changing. The work of Alexander Mikhailovich Lyapunov, who presented definitions and theorems for 
studying the stability of solutions to a broad class of differential equations, has been used extensively to address this problem [16]. The work of Lyapunov relies on defining an energy function, formally known as a Lyapunov function candidate, that can be used to determine the stability of a system without having to solve for the solutions to the system explicitly. Originally, this Lyapunov function was purely the total mechanical or electrical energy and therefore by nature positive definite. This was proven to be a necessary condition for the Lyapunov function candidate.

\section{Autonomous System Stability Analysis}

For autonomous systems, if the derivative of the Lyapunov function candidate was negative definite this meant that the system's energy would dissipate and eventually

converge to an equilibrium point asymptotically if the initial state began close enough to the equilibrium point. If the Lyapunov function was radially unbounded it meant that global asymptotic stability was possible. However, finding a suitable Lyapunov function can be a challenge as well as obtaining a negative definite derivative of the energy function. The former problem can be addressed by careful observation of a system's physical properties, the variable gradient method, or Krasovskii's method [27]. The latter problem can be addressed through the use of invariant set theorems.

The invariant set theorems are useful in determining whether asymptotic stability can be achieved when the derivative of the Lyapunov function candidate is negative semidefinite. Concepts taken from LaSalle's invariance principle allow for the extension of the concept of dissipating energy that the Lyapunov function represents so that convergence to an equilibrium or, in some cases, a limit cycle can 
be determined [27]. Basically, if a Lyapunov function can be found, its derivative is negative semidefinite, and if no trajectory other than the equilibrium point can keep the Lyapunov functions derivative equal to zero, then the corresponding equilibrium point is asymptotically stable [23].

\section{Non-Autonomous System Stability Analysis}

For non-autonomous systems, the above description of the Lyapunov stability analysis for autonomous systems can be similarly applied with the addition of some concepts such as decrescent functions and uniformity. In this context, decrescent functions are positive definite functions explicitly dependent on time but always less than a time-invariant positive definite function. Likewise, under these circumstances, uniformity in a system's behavior means that a system will behave similarly independent of an initial time, $t_{0}$. However, the concepts taken from LaSalle's invariance principle do not apply. This can be partially overcome by Barbalat's Lemma. Before intoducing the lemma it is important to first introduce signal norms that will aid in the discussion of the Barbalat lemma as well as the Gronwall-Bellman lemma, which are vital to stability analysis. Though it is not of great importance to this thesis, the

Lefschetz-Kalman-Yakubovich lemma embodies essential concepts used in adaptive control design and analysis and will be discussed as well.

Signal Norms For a vector signal, the $L^{1}, L^{2}$, and $L^{\infty}$ norms are defined as 


$$
\begin{aligned}
\|x(\cdot)\|_{1} & =\int_{0}^{\infty}\|x(t)\|_{1} d t \\
\|x(\cdot)\|_{2} & =\sqrt{\int_{0}^{\infty}\|x(t)\|_{2}^{2} d t} \\
\|x(\cdot)\|_{\infty} & =\sup _{t \geq 0}\|x(t)\|_{\infty}
\end{aligned}
$$

where the vector signal norms are

$$
\begin{aligned}
\|x(t)\|_{1} & =\left|x_{1}(t)\right|+\cdots+\left|x_{n}(t)\right|, \\
\|x(t)\|_{2} & =\sqrt{x_{1}^{2}(t)+\cdots+x_{n}^{2}(t)}, \\
\|x(t)\|_{\infty} & =\max _{1 \leq i \leq n}\left|x_{i}(t)\right| .
\end{aligned}
$$

Barbalat Lemma The Barbalet lemma can be used to draw conclusions about the asymptotic properties of functions and their derivatives. It states that if a scalar function $f(t)$ is uniformly continuous, such that $\lim _{t \rightarrow \infty} \int_{0}^{t} f(\tau) d \tau$ exists and is finite then $\lim _{t \rightarrow \infty} f(t)=0$. Basically, the $L^{1}$ norm is used to determine if $\lim _{t \rightarrow \infty} \int_{0}^{t} f(\tau) d \tau$ exists and is finite. The $L^{\infty}$ norm determines if $\dot{f}(t)$ is bounded, which is a sufficient condition to say that $f(t)$ is uniformly continuous. With the knowledge that if a signal $f(t) \in L^{1} \cap L^{\infty} \Rightarrow f(t) \in L^{2}$, where the $L^{2}$ norm verifies whether $f(t)$ has finite energy, we can say that $\lim _{t \rightarrow \infty} f(t)=0$. The Barbalat lemma is particularly useful in the analysis of non-autonomous systems since it can be difficult to find a Lyapunov function candidate with a negative definite derivative [20] [27]. 
Gronwall-Bellman Lemma Often it is not easy to show that a signal is bounded. Boundedness of certain signals is critical when using the Barbalat lemma as well as for other situations, such as knowing the boundedness of signals in the presence of disturbances [27]. The Gronwall-Bellman lemma is used to prove that a signal is bounded. The lemma states that if some functions $f(t), g(t)$, and $k(t)$ are continuous, where $g(t) \geq 0, k(t) \geq 0$, for all $t \geq t_{0}$ and for some $t_{0} \geq 0$ there is a continuous function $u(t)$ that satisfies $u(t) \leq f(t)+g(t) \int_{t_{0}}^{t} k(\tau) u(\tau) d \tau$ for all $t \geq t_{0}$ then $u(t) \leq f(t)+g(t) \int_{t_{0}}^{t} k(\tau) f(\tau) e^{\int_{\tau}^{t} k(\sigma) g(\sigma) d \sigma} d \tau$. In the previous integral inequalities the function $u(t)$ is the signal of interest. To reiterate, this lemma allows for an explicit bound to be determined on $u(t)$ [20] [27].

Lefschetz-Kalman-Yakubovich Lemma A way of testing for strictly positive realness is provided by the Lefschetz-Kalman-Yakubovich lemma. This concept is important when defining Lyapunov function candidates or error functions since it is essential to prove that they are positive definite. The three criteria for a transfer function, $h(s)$, to be strictly positive real is that it must first be analytic in $R e[s] \geq 0$. Second, it must be that $\operatorname{Re}[h(j \omega)]>0$ for all $\omega \in(-\infty, \infty)$. Finally, the third criterion is that if $n^{*}=1$, where $n^{*}$ is the relative degree of the transfer function $h(s)$, then $\lim _{\omega^{2} \rightarrow \infty} \omega^{2} R e[h(j \omega)]>0$. If $n^{*}=0$ then $\lim _{\omega \rightarrow \infty} R e[h(j \omega)]>0$. Finally, if $n^{*}=-1$ then $\lim _{\omega \rightarrow \infty} \operatorname{Re}[h(j \omega)]>0$ and $\lim _{\omega \rightarrow \infty} \frac{\operatorname{Re}[h(j \omega)]}{j \omega}>0$ [20]. 


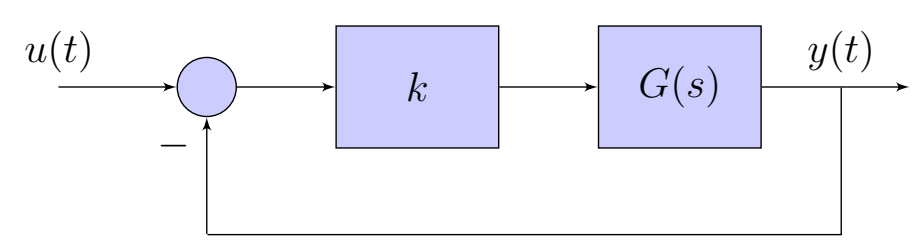

Figure 2.1: Block diagram of a system with output feedback and an adjustable preamplifier gain $k$.

\subsection{Classical Control}

In the case that a classical control system model is unstable it can be made stable by introducing feedback. Classically, the output $y(t)$ is fed back and compared to the system input $u(t)$. There are a few options available for control with this configuration that include gain adjustment, lag-compensation, lead-compensation, and lag-lead compensation. An example of a control system setup for gain adjustment with gain $k$ and the system transfer function $G(s)$ is shown in fig. 2.1.

With the state space representation it is convenient to instead feed back the state variables to what is now the control signal, $u(t)$, instead of an input. With this configuration each state variable can be adjusted by a gain vector $K$ to give the desired closed loop poles. A typical control system represented with the state space representation utilizing state feedback is displayed in fig. 2.2 [45].

\subsection{Adaptive Control}

Adaptive control differs from classical control in that instead of having fixed gains that are chosen or designed for some desired performance they are instead updated 


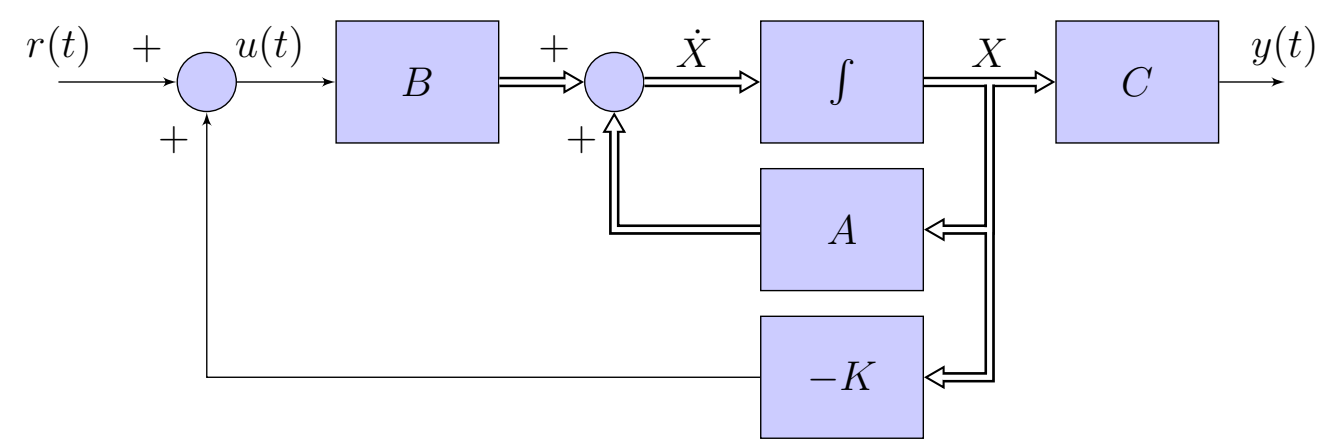

Figure 2.2: State space representation of a plant with state feedback.

online. This allows the control system to modify its control laws to account for changes to the plant parameters that are unknown or unable to be prevented. The closed-loop system can then adjust itself towards an optimal operating condition that still achieves the desired system performance. Since an adaptive control system has this capability it is possible for the system to operate far beyond that which is possible with a classical control system.

There are two approaches to adaptive control design. The first is known as Direct Adaptive Control and is characterized by the ability to update the controller parameters online through the use of an adaptive update law without initially determining the characteristics of the plant and the possible disturbances. The second is known as Indirect Adaptive Control and is characterized by first estimating the parameters of the plant being controlled as well as the possible disturbances, then updating the controller based on an adaptive update law. Direct adaptive control algorithms will be employed in this thesis. 


\section{Chapter 3}

\section{Aircraft Models with Icing}

The models to be discussed are that which were developed by Amanda Lampton and John Valasek of Texas A\&M University and are based partly on the work of Bragg et al [34] [32] [12]. The work presented in [4], [3], and [5] details a method to model airplanes with ice accretion for which no ice accretion data either exists or is available for use. It accounts for changes in dynamic response due to unequally distributed icing levels and severity between the wing, the horizontal tail, and the full aircraft. In this model it is assumed that differing levels of ice on the wing and tail appear as changes in the lift and drag force of a lifting surface which in turn leads to a decrease in stability and control [3].

Most of the data on the effects of ice accretion on the parameters of an airplane have been obtained through the study of the DeHavilland Twin Otter [32]. Since this is the case, the Twin Otter data was used in the development of this model. State space models describing both the longitudinal and lateral-directional equations of 


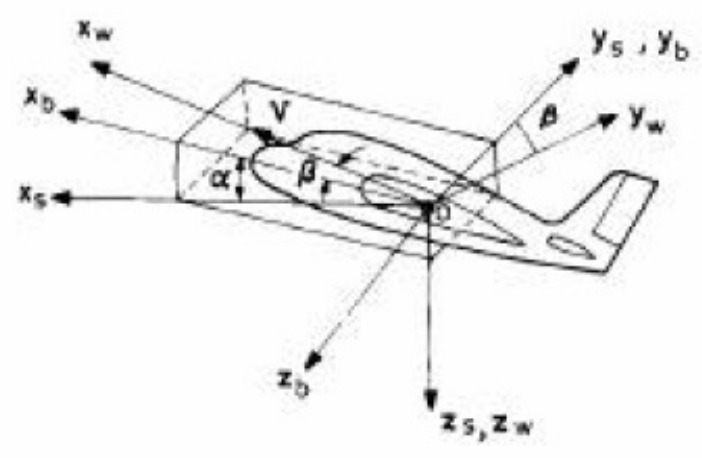

Figure 3.1: Body, wind, and stability axis systems and aerodynamic angles. Reproduced from [8].

motion for a Cessna 208 Super Cargomaster will be presented with the appropriate icing models, based on the Twin Otter data, applied. Figure 3.1 is shown as a reference to demonstrate the stability axis system coordinates used in the development of the equations of motion [3] [4].

\subsection{Physics of Icing}

It is imperative to understand the consequences of ice buildup on the surfaces of aircraft to appreciate the potential for disaster inherent in such a situation. From Archimedes principle, an aircraft will experience a buoyancy force equal to the weight of air displaced by it. Lift is generated by all surfaces of the airplane. However, the wings generate most of it. For most wings, a great deal of the lift is created just aft of the leading edge of the airfoil. As the air flows over an aircraft's wing in flight the speed of the air under the wing is slower than the speed of the air over the wing. As a result, the pressure under the wing is higher than the pressure over the wing. 
This difference in pressure on either side of the lifting surface is what creates lift. The shape of the airfoil governs the amount of pressure experienced on either side of it. Thus, any changes to the shape result in changes to the aerodynamic forces the plane experiences [15].

\section{Forms of Ice Accretion}

For ice to form there are certain atmospheric conditions that must be present. These conditions are either noticed by the flight crew or brought to their attention by the local air traffic controller. Atmospheric conditions that are conducive to ice accretion exist when visible moisture of any kind is present and either the outside air temperature on the ground is less than $5^{\circ} \mathrm{C}\left(41^{\circ} \mathrm{F}\right)$, or the air temperature in flight is less than $7^{\circ} \mathrm{C}\left(44.6^{\circ} \mathrm{F}\right)[40]$. The Federal Aviation Administration has strict guidelines on the actions that should be taken by the flight crew to avoid ice buildup on the aircraft. Despite this, ice accretions can still occur.

In general, there are three types of ice accretions that will form on an airplane. These are commonly referred to as rime, glaze, and mixed ice. Rime ice is characterized by a rough, milky white appearance, and, in general, grows into large accretions on the airfoil. Much of it can be removed by de-icing systems or prevented by anti-icing systems. Glaze ice is characterized by a clear and smooth appearance. However, if pockets of air are present during the freezing process it will cause this type of ice accretion to become lumpy. As the ice accretion grows, glaze ice has a tendency to form a horn of ice. A horn of ice is any ice accretion that grows large enough in size to cause a significant change to the shape of the airfoil. The size of an 


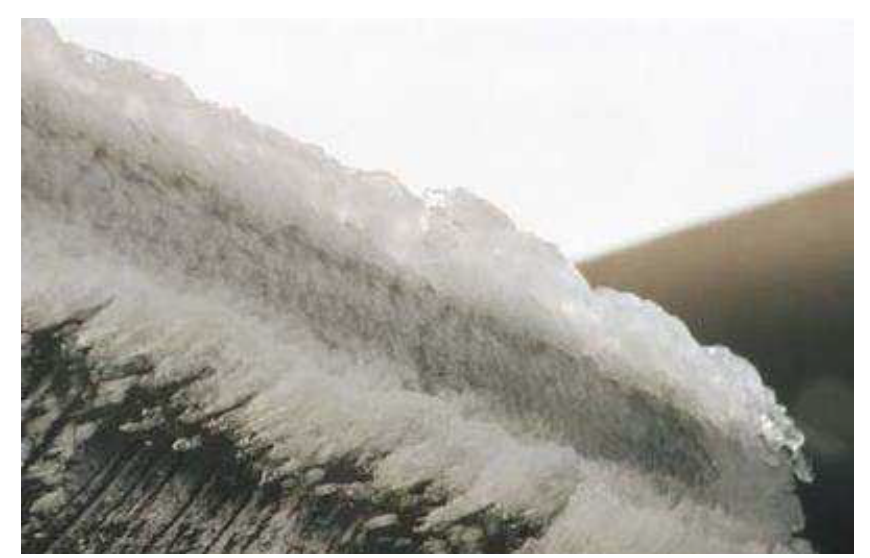

Figure 3.2: Severe mixed ice accumulation on the tail of a full-scale NASA test aircraft. Reproduced from [56].

ice accretion required to make this change is largely dependent on the shape of the airfoil. According to [7] "wind tunnel and flight tests have shown that frost, snow, and ice accumulations (on the leading edge or upper surface of the wing) no thicker or rougher than a piece of coarse sandpaper can reduce lift by $30 \%$ and increase drag up to $40 \%$ ". It has been observed that in some cases larger ice accretions can reduce the lift further and increase drag up to $80 \%$ or more. Finally, mixed ice is a combination of rime and glaze ice and is also likely to form a horn of ice as the ice accretion grows in size [7]. A severe case of mixed ice is depicted in fig. 3.2.

\section{Aerodynamic Effects of Ice Accretion}

With ice accumulating along the leading edge of lifting surfaces, the resultant aerodynamic effects are detrimental to the performance of the aircraft. That being said, ice of any kind will increase drag, decrease the total lift, and decrease the static longitudinal stability. It is true that there are de-icing devices, such as de-icing boots 
and heating strips, installed on all planes to prevent ice accumulation. However, this is not always a solution to the problem. Malfunctions of de-icing systems can cause the build up of ice aft of the devices. In some cases ice accumulates so quickly that it will build up between cycles of the de-icing systems and they will be unable to remove all of the ice accumulated. Worse still, the de-icing systems could cease to operate at all. The type of ice and the severity of which it accumulates is highly dependent on the velocity of the airplane, exposure time, atmospheric air temperature, liquid water content, and the median volumetric diameter of the liquid drops [3].

For most wings, a great deal of the lift is created just aft of the leading edge. When ice accumulates, it disrupts the air flowing across the airfoil. As stated before, this disruption is usually caused by a horn of ice which creates a separation bubble, depicted in fig. 3.3. With the separation bubble, the lift contribution from the leading edge of the wing is greatly reduced causing a reduction in the surface pressure on the trailing edge aileron, elevator, or other flap present on the aircraft. The trailing edge of the wing now has a negative hinge moment, or in other words the trailing edge rises before the leading edge, and a change in stick force is felt by the pilot. In severe cases this can cause aileron hinge moment reversal, aileron snatch, or both [34].

\section{Aileron Hinge Moment Reversal and Aileron Snatch}

Aileron hinge moment reversal is experienced when the separation bubble due to a horn of ice has increased with angle of attack to a point that it has created a low pressure region over the aileron. This low pressure remains once the aileron changes position from, for example, being down, which causes an increase in lift, to up, which 


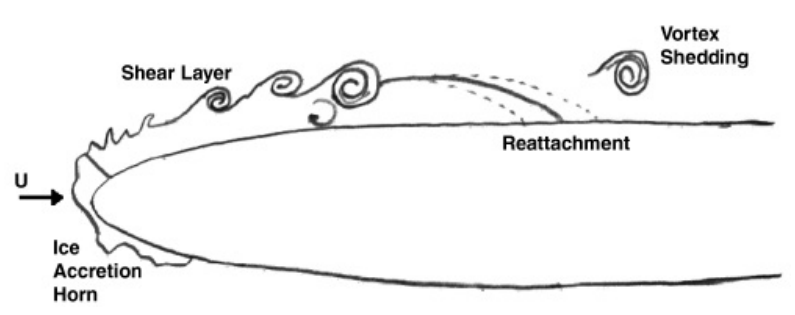

Figure 3.3: Upper surface separation bubble aft of leading-edge ice accretion. Reproduced from [22].

would normally cause a decrease in lift. The hinge moment about the aileron would remain as it was before because the pressures on either side of the wing have remained the same. Basically, instead of the expected roll angle due to the deflection of the ailerons the roll angle would be opposite of that which was expected. The severity of the change in roll angle would be largely dependent on the airspeed, angle of attack, and ice accumulation on other surfaces of the aircraft. Since this behavior is opposite of that under ideal conditions the phenomenon is referred to as aileron hinge moment reversal [34].

Aileron snatch results from the hinge moments being altered to such an extent that the aileron is pulled up by low pressure on the top with sufficient force as to induce an uncommanded roll [34]. Cases where aileron snatch is the cause of an uncommanded role may be described as aileron hinge moment reversal since it is a special case of aileron hinge moment reversal. The above discussion can be extended to the elevators and rudder of an airplane. It is also important to note that as the aileron and rudder deflection angles are changed the resultant rolling and yawing moments increase [3] [5] [34]. 


\section{Wing Stall and Tail Stall}

With sufficiently large ice accretions, wing stall and tail stall will occur at much lower angles of attack than it would if the airplane was clean [7]. This early stall is caused by a seperation bubble formed from the disruption of airflow by a horn of ice just as in the above examples of aileron hinge moment reversal and snatch. The difference is that ice has formed along a majority of the wing now instead of with respect to just the ailerons.

Tail stall is particularly dangerous as it is usually unexpected, since the flight crew, in most cases, has no visual contact with the tail, and the stall causes the airplane to immediately pitch downward as there is no longer enough pressure forcing the tail down. It has been reported that ice accretions on the tail of aircraft can grow three to six times thicker than those on the wings. This is due to the smaller leading edge radius of the tail relative to the wings [56].

\subsection{Aircraft Models without Icing}

In this section the equations of motion without icing for both the longitudinal and lateral-directional dynamics will be presented. Following that discussion the resultant state space models for the longitudinal and lateral-directional dynamics will be given. 


\subsubsection{Equations of Motion without Icing}

The equations of motion for an aircraft are derived from the resultant equations of Newton's second law

$$
\begin{aligned}
\text { Force } & =\text { Mass } \times \text { Acceleration } \\
\text { Moment } & =\text { Moment of Inertia } \times \text { Angular Acceleration }
\end{aligned}
$$

In this model the equations are found with respect to the stability axis coordinate system of fig. 3.1.

\section{Assumptions}

The major assumptions made in this model are

(A3.1) the aircraft is maintaining level 1-g trimmed flight in the stability axis system; this means that the steady state aircraft body axis roll rate $P_{1}$, body axis pitch rate $Q_{1}$, body axis yaw rate $R_{1}$, velocity in the body axis $z$ direction $W_{1}$, velocity in the body axis $y$ direction $V_{1}$, and roll attitude angle $\Phi_{1}$ are all equal to zero;

(A3.2) the pitch attitude angle $\Theta_{1}$ is a constant;

(A3.3) the aircraft is assumed to be rigid, meaning that no bending of the airframe due to exterior forces occurs.

The steady state or trim condition is the equilibrium condition defined by the pitching moment about the center of gravity being equal to zero [8]. 
It is also of importance to note that the notation to be used for the equations of motion makes use of the American normalized dimensional derivative notation. An example of this notation is $X_{u}$, which is the linear acceleration in the body axis $x$ direction per unit change in the velocity in the stability axis $x$ direction $u$. This is the same as $\frac{\stackrel{\circ}{X}_{u}}{m}$, where $\stackrel{\circ}{X}_{u}$ is the dimensional derivative notation and is equal

to $\frac{\partial X}{\partial u}$. This notation, $X_{u}=\frac{\stackrel{\circ}{X_{u}}}{m}$, is the reason the mass $m$ does not appear in the equations to be presented. More details regarding the differences between notation can be found in [36]. For further explanation on the stability axis system and how the equations of motion can be derived see [8], [26], [16], and [36].

\section{Longitudinal Equations of Motion}

Using (3.1), and its associated physical principles, the following longitudinal small perturbation equations of motion of the coupled system in the stability axis, derived in [3], are defined using Taylor series expansions as

$$
\begin{aligned}
\dot{u}= & -g \theta \cos \Theta_{1}+\left(X_{T_{u}}+X_{u}\right) u+X_{\alpha} \alpha+X_{q} q \\
& +X_{\delta_{e}} \delta_{e}+X_{\delta_{T}} \delta_{T}+X_{\dot{\alpha}} \dot{\alpha} \\
\dot{\alpha}= & \frac{\dot{\omega}}{U_{1}}=\left(-g \theta \sin \Theta_{1}+Z_{u} u+Z_{\alpha} \alpha+Z_{q} q+U_{1} q\right. \\
& \left.+Z_{\delta_{e}} \delta_{e}+Z_{\delta_{T}} \delta_{T}+Z_{\dot{\alpha}} \dot{\alpha}\right) / U_{1} \\
\dot{q}= & \left(M_{T_{u}}+M_{u}\right) u+\left(M_{T_{\alpha}}+M_{\alpha}\right) \alpha+M_{q} q+M_{\delta_{e}} \delta_{e} \\
& +M_{\delta_{T}} \delta_{T}+M_{\dot{\alpha}} \dot{\alpha} \\
\dot{\theta}= & q .
\end{aligned}
$$


For the $\dot{u}$ equation, $\dot{u}$ is the incremental change in the stability axis airplane velocity in the $x$ direction, $g$ is the gravitational acceleration, $\theta$ is the pitch attitude angle, $X_{T_{u}}$ is the linear acceleration in the body axis $x$ direction per unit change in speed due to thrust, $X_{u}$ is the linear acceleration in the body axis $x$ direction per unit change in the velocity in the stability axis $x$ direction $u, u$ is the stability axis airplane velocity in the $x$ direction, $X_{\alpha}$ is the linear acceleration in the body axis $x$ direction per unit change in the angle of attack $\alpha, \alpha$ is the angle of attack, $X_{q}$ is the linear acceleration in the body axis $x$ direction per unit change in the perturbed airplane body axis pitch rate $q, q$ is the perturbed airplane body axis pitch rate, $X_{\delta_{e}}$ is the linear acceleration in the body axis $x$ direction per unit change in elevator deflection angle $\delta_{e}, \delta_{e}$ is the elevator deflection angle, $X_{\delta_{T}}$ is the linear acceleration in the body axis $x$ direction per unit change in thrust $\delta_{T}, \delta_{T}$ is the change in thrust, $X_{\dot{\alpha}}$ is the linear acceleration in the body axis $x$ direction per unit change in the incremental change in angle of attack, and $\dot{\alpha}$ is the incremental change in angle of attack.

For the $\dot{\alpha}$ equation, $\dot{\alpha}$ is the incremental change in angle of attack, $\dot{\omega}$ is the incremental change in the stability axis aircraft velocity in the $z$ direction, $U_{1}$ is the airplane velocity in the body-axis $x$ direction, $Z_{u}$ is the linear acceleration in the body axis $z$ direction per unit change in the stability axis airplane velocity in the $x$ direction $u, Z_{\alpha}$ is the linear acceleration in the body axis $z$ direction per unit change in the angle of attack $\alpha, Z_{q}$ is the linear acceleration in the body axis $z$ direction per unit change in the perturbed airplane body axis pitch rate $q, Z_{\delta_{e}}$ is the linear acceleration in the body axis $z$ direction per unit change in the elevator deflection 
angle $\delta_{e}, Z_{\delta_{T}}$ is the linear acceleration in the body axis $z$ direction per unit change in the thrust $\delta_{T}$, and $Z_{\dot{\alpha}}$ is the linear acceleration in the body axis $z$ direction per unit change in the incremental change in angle of attack $\dot{\alpha}$.

For the $\dot{q}$ equation, $\dot{q}$ is the airplane body axis pitch acceleration, $M_{T_{u}}$ is the incremental change in the pitch angular acceleration per unit change in speed due to thrust, $M_{u}$ is the incremental change in the pitch angular acceleration per unit change in the stability axis airplane velocity in the $x$ direction $u, M_{T_{\alpha}}$ is the incremental change in the pitch angular acceleration per unit change in speed due to angle of attack, $M_{\alpha}$ is the incremental change in the pitch angular acceleration per unit change in the angle of attack $\alpha, M_{q}$ is the incremental change in the pitch angular acceleration per unit change in the perturbed airplane body axis pitch rate $q, M_{\delta_{e}}$ is the incremental change in the pitch angular acceleration per unit change in the

elevator deflection angle $\delta_{e}, M_{\delta_{T}}$ is the incremental change in the pitch angular acceleration per unit change in the thrust $\delta_{T}$, and $M_{\dot{\alpha}}$ is the incremental change in the pitch angular acceleration per unit change in the incremental change in angle of attack $\dot{\alpha}$.

For the $\dot{\theta}$ equation, $\dot{\theta}$ is the incremental change in pitch attitude angle.

\section{Lateral-Directional Equations of Motion}

Using (3.1), and its associated physical principles, the following lateral-directional small perturbation equations of motion of the coupled system in the stability axis, derived in [4], are defined using Taylor series expansions as 


$$
\begin{aligned}
\dot{p}= & L_{\beta} \beta+L_{p} p+L_{r} r+L_{\delta_{a}} \delta_{a}+L_{\delta_{R}} \delta_{R}+\frac{I_{x z}}{I_{x x}} \dot{r} \\
\dot{\phi}= & p+r \tan \Theta_{1} \\
\dot{\beta}= & \left(Y_{p} p+g \phi \cos \Theta_{1}+Y_{\beta} \beta+\left(Y_{r}-U_{1}\right) r\right. \\
& \left.+Y_{\delta_{a}} \delta_{a}+Y_{\delta_{R}} \delta_{R}\right) / U_{1} \\
\dot{r}= & N_{\beta} \beta+N_{p} p+N_{r} r+N_{\delta_{a}} \delta_{a}+N_{\delta_{R}} \delta_{R}+\frac{I_{x z}}{I_{z z}} \dot{p} \\
\dot{\psi}= & r \sec \Theta_{1} .
\end{aligned}
$$

For the $\dot{p}$ equation, $\dot{p}$ is the aircraft body axis roll acceleration, $L_{\beta}$ is the roll angular acceleration per unit change in the sideslip angle $\beta, \beta$ is the sideslip angle, $L_{p}$ is the roll angular acceleration per unit change in the perturbed aircraft body axis roll rate $p, p$ is the perturbed aircraft body axis roll rate, $L_{r}$ is the roll angular acceleration per unit change in the perturbed aircraft body-axis yaw rate $r, r$ is the perturbed aircraft body-axis yaw rate, $L_{\delta_{a}}$ is the roll angluar acceleration per unit change in the aileron deflection angle $\delta_{a}, \delta_{a}$ is the aileron deflection angle, $L_{\delta_{R}}$ is the roll angluar acceleration per unit change in the rudder deflection angle $\delta_{R}, \delta_{R}$ is the rudder deflection angle, $I_{x z}$ is the airplane moment of inertia about the body $x z$ plane, $I_{x x}$ is the airplane moment of inertia about the body $x$ axis, and $\dot{r}$ is the aircraft body-axis yaw acceleration.

For the $\dot{\phi}$ equation, $\dot{\phi}$ is the incremental change in roll attitude angle, and $r$ is the perturbed aircraft body-axis yaw rate.

For the $\dot{\beta}$ equation, $\dot{\beta}$ is the incremental change in sideslip angle, $Y_{p}$ is the linear 
acceleration in the body axis $y$ direction per unit change in the perturbed aircraft body axis roll rate $p, Y_{\beta}$ is the linear acceleration in the body axis $y$ direction per unit change in the sideslip angle $\beta, \beta$ is the sideslip angle, $Y_{r}$ is the linear acceleration in the body axis $y$ direction per unit change in the perturbed aircraft body-axis yaw rate $r, Y_{\delta_{a}}$ is the linear acceleration in the body axis $y$ direction per unit change in the aileron deflection angle $\delta_{a}$, and $Y_{\delta_{R}}$ is the linear acceleration in the body axis $y$ direction per unit change in the rudder deflection angle $\delta_{R}$.

For the $\dot{r}$ equation, $\dot{r}$ is the aircraft body-axis yaw acceleration, $N_{\beta}$ is the yaw angular acceleration per unit change in the sideslip angle $\beta, N_{p}$ is the yaw angular acceleration per unit change in the perturbed aircraft body axis roll rate $p, N_{r}$ is the yaw angular acceleration per unit change in the perturbed aircraft body-axis yaw rate $r, N_{\delta_{a}}$ is the yaw angular acceleration per unit change in the aileron deflection angle $\delta_{a}, N_{\delta_{R}}$ is the yaw angular acceleration per unit change in the rudder deflection angle $\delta_{R}$, and $I_{z} z$ is the airplane moment of inertia about the body $z$ axis.

For the $\dot{\psi}$ equation, $\dot{\psi}$ is the incremental change in heading angle.

\subsubsection{State Space Models without Icing}

Now that the system of equations developed in [4] and [3] describing an aircraft in no icing conditions have been presented, the set of equations (3.2) and (3.3) are then converted into matrix form, commonly referred to as a state space model. A state space representation of a linear time invariant system is used to determine the response of the system to any arbitrary input. Only slow maneuvers of the aircraft are considered with the result being that the parameters of the system change 
slowly enough to allow time invariance of the model to be assumed [3] [4]. For more information on the procedures used to create state descriptions of dynamic systems see references [36], [18], and [45].

\section{Longitudinal Dynamic Model}

To begin, the state variables of equation (3.2) are defined in [3] as

$$
\mathbf{x}(\mathbf{t})=\left[\begin{array}{llll}
u & \alpha & q & \theta
\end{array}\right]^{T}
$$

The inputs are defined to be

$$
\mathbf{u}(\mathbf{t})=\left[\begin{array}{l}
\delta_{e} \\
\delta_{T}
\end{array}\right] .
$$

The procedure for obtaining the system matrix $A$ and input matrix $B$ from equation (3.2) is detailed in [3]. They are presented here as

$$
A=\left[\begin{array}{cccc}
X_{u}^{\prime} & X_{\alpha}^{\prime} & X_{q}^{\prime} & X_{\theta}^{\prime} \\
Z_{u}^{\prime} & Z_{\alpha}^{\prime} & Z_{q}^{\prime} & Z_{\theta}^{\prime} \\
M_{u}^{\prime} & M_{\alpha}^{\prime} & M_{q}^{\prime} & M_{\theta}^{\prime} \\
0 & 0 & 1 & 0
\end{array}\right]
$$




$$
B=\left[\begin{array}{cc}
X_{\delta_{e}}^{\prime} & X_{\delta_{T}}^{\prime} \\
Z_{\delta_{e}}^{\prime} & Z_{\delta_{T}}^{\prime} \\
M_{\delta_{e}}^{\prime} & M_{\delta_{T}}^{\prime} \\
0 & 0
\end{array}\right]
$$

where the primed notation denotes the modified American derivative. For this research the outputs of interest are the states. Therefore, the output matrix $C$ is chosen to be

$$
C=\left[\begin{array}{llll}
1 & 0 & 0 & 0 \\
0 & 1 & 0 & 0 \\
0 & 0 & 1 & 0 \\
0 & 0 & 0 & 1
\end{array}\right]
$$

Finally, the matrices $A, B$, and $C$ along with the vectors $x(t)$ and $u(t)$ are combined into the state space form

$$
\left[\begin{array}{c}
\dot{u} \\
\dot{\alpha} \\
\dot{q} \\
\dot{\theta}
\end{array}\right]=\left[\begin{array}{cccc}
X_{u}^{\prime} & X_{\alpha}^{\prime} & X_{q}^{\prime} & X_{\theta}^{\prime} \\
Z_{u}^{\prime} & Z_{\alpha}^{\prime} & Z_{q}^{\prime} & Z_{\theta}^{\prime} \\
M_{u}^{\prime} & M_{\alpha}^{\prime} & M_{q}^{\prime} & M_{\theta}^{\prime} \\
0 & 0 & 1 & 0
\end{array}\right]\left[\begin{array}{l}
u \\
\alpha \\
q \\
\theta
\end{array}\right]+\left[\begin{array}{cc}
X_{\delta_{e}}^{\prime} & X_{\delta_{T}}^{\prime} \\
Z_{\delta_{e}}^{\prime} & Z_{\delta_{T}}^{\prime} \\
M_{\delta_{e}}^{\prime} & M_{\delta_{T}}^{\prime} \\
0 & 0
\end{array}\right]\left[\begin{array}{c}
\delta_{e} \\
\delta_{T}
\end{array}\right]
$$




$$
\left[\begin{array}{l}
u \\
\alpha \\
q \\
\theta
\end{array}\right]=\left[\begin{array}{llll}
1 & 0 & 0 & 0 \\
0 & 1 & 0 & 0 \\
0 & 0 & 1 & 0 \\
0 & 0 & 0 & 1
\end{array}\right]\left[\begin{array}{l}
u \\
\alpha \\
q \\
\theta
\end{array}\right]
$$

The model (3.9) and (3.10) reflects the Cessna 208 Super Cargomaster with no ice accumulated on the aircraft. The following numerical values, obtained from [3], reflect the aircraft flying at 15,000 feet at a speed of 135 knots. These values will be used later in this thesis to determine an aircraft model in icing conditions. That being said, the numerical values for the state space model (3.9) are presented as,

$$
\left[\begin{array}{c}
\dot{u} \\
\dot{\alpha} \\
\dot{q} \\
\dot{\theta}
\end{array}\right]=\left[\begin{array}{cccc}
-0.03 & 17.74 & 0 & -32.17 \\
-0.0013 & -1.17 & 0.97 & -0.0031 \\
-0.0005 & -8.71 & -1.79 & 0.0003 \\
0 & 0 & 1 & 0
\end{array}\right]\left[\begin{array}{l}
u \\
\alpha \\
q \\
\theta
\end{array}\right]+\left[\begin{array}{cc}
-0.55 & 2.79 \\
-0.11 & 0 \\
-8.78 & 0 \\
0 & 0
\end{array}\right]\left[\begin{array}{c}
\delta_{e} \\
\delta_{T}
\end{array}\right] .
$$

\section{Lateral-Directional Dynamic Model}

To begin, the state variables of equation (3.3) are defined in [4] as

$$
\mathbf{x}(\mathbf{t})=\left[\begin{array}{lllll}
p & \phi & \beta & r & \psi
\end{array}\right]^{T},
$$

The inputs are defined to be 


$$
\mathbf{u}(\mathbf{t})=\left[\begin{array}{l}
\delta_{a} \\
\delta_{R}
\end{array}\right] .
$$

The procedure for obtaining the system matrix $A$ and input matrix $B$ from equation (3.3) is detailed in [4]. They are presented here as

$$
\begin{gathered}
A=\left[\begin{array}{ccccc}
L_{p}^{\prime} & 0 & L_{\beta}^{\prime} & L_{r}^{\prime} & 0 \\
1 & 0 & 0 & \tan \Theta_{1} & 0 \\
\frac{Y_{p}}{U_{1}} & \frac{g \cos \Theta_{1}}{U_{1}} & \frac{Y_{\beta}}{U_{1}} & \left(\frac{Y_{r}}{U_{1}}-1\right) & 0 \\
N_{p}^{\prime} & 0 & N_{\beta}^{\prime} & N_{r}^{\prime} & 0 \\
0 & 0 & 0 & \sec \Theta_{1} & 0
\end{array}\right] \\
B=\left[\begin{array}{cc}
L_{\delta_{a}}^{\prime} & L_{\delta_{R}}^{\prime} \\
0 & 0 \\
\frac{Y_{\delta_{a}}}{U_{1}} & \frac{Y_{\delta_{R}}}{U_{1}} \\
N_{\delta_{a}}^{\prime} & N_{\delta_{R}}^{\prime} \\
0 & 0
\end{array}\right]
\end{gathered}
$$

where the primed notation denotes the modified American derivative. For this research the outputs of interest are the states. Therefore, the output matrix $C$ is chosen to be 


$$
C=\left[\begin{array}{lllll}
1 & 0 & 0 & 0 & 0 \\
0 & 1 & 0 & 0 & 0 \\
0 & 0 & 1 & 0 & 0 \\
0 & 0 & 0 & 1 & 0 \\
0 & 0 & 0 & 0 & 1
\end{array}\right]
$$

Finally, the matrices $A, B$, and $C$ along with the vectors $x(t)$ and $u(t)$ are combined into the state space form

$$
\left[\begin{array}{c}
\dot{p} \\
\dot{\phi} \\
\dot{\beta} \\
\dot{r} \\
\dot{\psi}
\end{array}\right]=\left[\begin{array}{ccccc}
L_{p}^{\prime} & 0 & L_{\beta}^{\prime} & L_{r}^{\prime} & 0 \\
1 & 0 & 0 & \tan \Theta_{1} & 0 \\
\frac{Y_{p}}{U_{1}} & \frac{g \cos \Theta_{1}}{U_{1}} & \frac{Y_{\beta}}{U_{1}} & \left(\frac{Y_{r}}{U_{1}}-1\right) & 0 \\
N_{p}^{\prime} & 0 & N_{\beta}^{\prime} & N_{r}^{\prime} & 0 \\
0 & 0 & 0 & \sec \Theta_{1} & 0
\end{array}\right]\left[\begin{array}{l}
p \\
\beta \\
\gamma \\
\gamma
\end{array}\right]+\left[\begin{array}{cc}
L_{\delta_{a}}^{\prime} & L_{\delta_{R}}^{\prime} \\
0 & 0 \\
\frac{Y_{\delta_{a}}}{U_{1}} & \frac{Y_{\delta_{R}}}{U_{1}} \\
N_{\delta_{a}}^{\prime} & N_{\delta_{R}}^{\prime} \\
0 & 0
\end{array}\right]\left[\begin{array}{c}
\delta_{a} \\
\delta_{R}
\end{array}\right]
$$

$$
\left[\begin{array}{l}
p \\
\phi \\
\beta \\
r \\
\psi
\end{array}\right]=\left[\begin{array}{lllll}
1 & 0 & 0 & 0 & 0 \\
0 & 1 & 0 & 0 & 0 \\
0 & 0 & 1 & 0 & 0 \\
0 & 0 & 0 & 1 & 0 \\
0 & 0 & 0 & 0 & 1
\end{array}\right]\left[\begin{array}{l}
p \\
\phi \\
\beta \\
\psi
\end{array}\right] .
$$

The model (3.17) and (3.18) reflects the Cessna 208 Super Cargomaster with no 
ice accumulated on the aircraft. The following numerical values, obtained from [4], reflect the aircraft flying at 15,000 feet at a speed of 135 knots. These values will be used later in this thesis to determine an aircraft model in icing conditions. That being said, the numerical values for the state space model (3.17) are presented as,

$$
\left[\begin{array}{c}
\dot{p} \\
\dot{\phi} \\
\dot{\beta} \\
\dot{r} \\
\dot{\psi}
\end{array}\right]=\left[\begin{array}{ccccc}
-4.10 & 0 & -5.96 & 0.77 & 0 \\
1 & 0 & 0 & 0.10 & 0 \\
-0.0014 & 0.14 & -0.17 & -0.99 & 0 \\
-0.19 & 0 & 2.16 & -0.52 & 0 \\
0 & 0 & 0 & 1 & 0
\end{array}\right]\left[\begin{array}{l}
p \\
\phi \\
\beta \\
r \\
\psi
\end{array}\right]+\left[\begin{array}{cc}
8.99 & 1.10 \\
0 & 0 \\
0 & 0.04 \\
-0.07 & -2.63 \\
0 & 0
\end{array}\right]\left[\begin{array}{c}
\delta_{a} \\
\delta_{R}
\end{array}\right] .
$$

\subsection{Aircraft Models with Icing}

In this section an icing effects model will be presented. It will then be applied to the longitudinal and lateral-directional equations of motion and state space models. Due to limtations on the information presented in [3] and [4] numerical values are only available for the state space models.

\subsubsection{Modeling Ice Accretion}

\section{Icing Effects Model}

To account for the effects of ice accretion, work developed by Bragg et al. was used. The work detailed in [32] and [12] presents an icing effects model applicable to any individual performance, stability, or control parameter or derivative effected 
by icing. The performance, stability, or control parameters or derivatives effected by icing are determined from,

$$
C_{(A)_{i c e d}}=\left(1+\eta_{i c e} k_{C_{(A)}}^{\prime}\right) C_{(A)}
$$

where $C_{(A)_{i c e d}}$ is the stability or control parameter or derivative with the effects of ice accretion taken into account, $C_{(A)}$ is the stability or control parameter or derivative, $\eta_{i c e}$ is the icing severity parameter, and $k_{C_{A}}^{\prime}$ is the coefficient icing factor that depends on the coefficient being modified and the aircraft specific information.

The aforementioned model proposed by Bragg et al. is not exceptionally accurate as there are still factors that contribute to ice accretion unaccounted for within it. Since this is the case, [3] and [4] define the product of $\eta_{i c e}$ and $k_{C_{(A)}}^{\prime}$ as an icing factor, $f_{i c e}$. This icing factor is calculated from data obtained from tests on a DeHavilland Twin Otter. Information regarding this calculation can be found in [4], [3], and [32]. The values of $f_{\text {ice }}$ relative to this model are reproduced from [3] and [4] in table 3.1. Substituting $f_{\text {ice }}$ into (3.20) gives

$$
C_{(A)_{i c e d}}=\left(1+f_{i c e}\right) C_{(A)}
$$

It should be noted that $C_{(A)_{i c e d}}$ and $C_{(A)}$ are dimensionless parameters. To dimensionalize the respective parameters for use, information specific to the airplane in question, such as the mean geometric chord and air density, would be needed.

It is important to remember that equation (3.21) can be applied to any individual performance, stability, or control parameter or derivative effected by icing. Therefore, 
the parameter $C_{(A)}$ in (3.20) refers to either the American normalized dimensional derivatives of equations (3.2) and (3.3) or the elements of the system matrix $A \in \mathbb{R}^{n \times n}$ and control matrix $B \in \mathbb{R}^{n \times m}$ of equations (3.9) and (3.17). The elements of the system matrix are referred to as stability parameters or derivatives while the elements of the control matrix are referred to as control parameters or derivatives. The use of either term in this case is interchangeable. In this work, the elements of matrices $A$ and $B$ will be referred to as the stability parameters and control parameters, respectively [36].

Table 3.1: Change in stability and control parameters $\left(f_{\text {ice }}\right)$ due to icing [3] [4].

\begin{tabular}{lr}
\hline \hline Longitudinal Parameters & $f_{\text {ice }}$ \\
\hline$\Delta Z_{0}$ & 0 \\
$\Delta Z_{\alpha}$ & -0.10 \\
$\Delta Z_{q}$ & -0.012 \\
$\Delta Z_{\delta_{e}}$ & -0.095 \\
$\Delta M_{0}$ & 0 \\
$\Delta M_{\alpha}$ & -0.099 \\
$\Delta M_{q}$ & -0.035 \\
$\Delta M_{\delta_{e}}$ & -0.10 \\
\hline Lateral-Directional Parameters & $f_{i c e}$ \\
\hline$\Delta Y_{\beta}$ & -0.20 \\
$\Delta Y_{\delta_{R}}$ & -0.08 \\
$\Delta L_{\beta}$ & -0.10 \\
$\Delta L_{p}$ & -0.10 \\
$\Delta L_{\delta_{a}}$ & -0.10 \\
$\Delta L_{\delta_{R}}$ & -0.08 \\
$\Delta N_{\beta}$ & -0.20 \\
$\Delta N_{r}$ & -0.061 \\
$\Delta N_{\delta_{a}}$ & -0.083 \\
\hline \hline
\end{tabular}




\section{Application to Parameters}

Applying (3.21) is relatively simple. For example, the parameter $M_{\alpha}$ from equation (3.2) in no icing conditions is

$$
M_{\alpha}=\frac{\bar{q} S \bar{c} C_{m_{\alpha}}}{I_{y y}}
$$

where $\bar{q}$ is the dynamic pressure, $S$ is the wing area, and $\bar{c}$ is the mean geometric chord of the aircraft. Applying (3.21) to (3.22) is equivalent to multiplying the entire right hand side of $(3.22)$ by the term $\left(1+f_{i c e}\right)$, where $f_{i c e}$ is the term $\Delta M_{\alpha}$ which can be found in table 3.1. Therefore, to find $M_{\alpha_{i c e d}}$, which is the parameter $M_{\alpha}$ with the effects of ice accretion taken into account, the equation (3.22) becomes

$$
M_{\alpha_{\text {iced }}}=\frac{\bar{q} S \bar{c}\left(1+f_{i c e}\right) C_{m_{\alpha}}}{I_{y y}} .
$$

Now, the application of (3.21) to the modified state space model parameters of the form $M_{\alpha}^{\prime}$ must be considered. There is no information presented in [4], [3], and [5] on how the American normalized dimensional derivatives are modified to become the American modified derivatives of the state space models. That being said, for the purposes of this research, the following assumption will be made.

(A3.4) The values listed in table 3.1, along with the $\left(1+f_{\text {ice }}\right)$ term, can be applied to both the American normalized dimensional derivatives of equations (3.2) and (3.3) or the elements of the system matrix $A \in \mathbb{R}^{n \times n}$ and input matrix $B \in \mathbb{R}^{n \times m}$ of equations (3.9) and (3.17). 
In this thesis all of the numerical values for the stability and control parameters given are dimensional. Therefore, the application of (3.21) to the equations of motion and the state space models can be written simply as,

$$
M_{\alpha_{i c e d}}=\left(1+f_{i c e}\right) M_{\alpha}
$$

for the equations of motion and,

$$
M_{\alpha_{\text {iced }}}^{\prime}=\left(1+f_{\text {ice }}\right) M_{\alpha}^{\prime}
$$

for the elements of the state space model's system matrix $A \in \mathbb{R}^{n \times n}$ and control matrix $B \in \mathbb{R}^{n \times m}$.

Modeling Equally Distributed Ice Accretion As an example of the use of (3.21) to account for ice accretion, consider the lateral-directional stability parameter $L_{\beta}^{\prime}$. Multiplying $L_{\beta}^{\prime}$ by $\left(1+f_{\text {ice }}\right)$, where $f_{\text {ice }}$ is the value $\Delta L_{\beta}$ from table 3.1 , gives

$$
L_{\beta_{\text {iced }}}^{\prime}=\left(1+\Delta L_{\beta}\right) L_{\beta}^{\prime}
$$

Performing the simple calculations needed gives the value of $L_{\beta_{i c e d}}^{\prime}$, which is the value of $L_{\beta}^{\prime}$ with a worst case scenario ice accretion present on the aircraft.

In equations like (3.26) differing levels of icing severity can be represented by multiplying $f_{\text {ice }}$ by a constant. For example,

$$
M_{\alpha_{\text {iced }}}^{\prime}=(1+(-0.099)) M_{\alpha}^{\prime}
$$


is a case where $f_{i c e}=\Delta M_{\alpha}=-0.099$ is multiplied by 1.0 and is the worst case scenario. To decrease the amount of ice affecting the parameter $M_{\alpha}^{\prime}$ you would multiply by a constant that is less than 1.0. For example,

$$
\begin{aligned}
& M_{\alpha_{\text {iced }}}^{\prime}=(1+0.2(-0.099)) M_{\alpha}^{\prime} \\
& M_{\alpha_{\text {iced }}}^{\prime}=(1+(-0.0198)) M_{\alpha}^{\prime}
\end{aligned}
$$

would mean that $M_{\alpha_{i c e d}}^{\prime}$ is made less stable than $M_{\alpha}^{\prime}$ by about $2 \%$ where before in (3.27) $M_{\alpha_{\text {iced }}}^{\prime}$ was made less stable than $M_{\alpha}^{\prime}$ by approximately $10 \%$.

As the previous example demonstrates, equation (3.21) is defined so that the stability and control parameters could be reduced by a certain percentage. However, it was found in [32] that there was a typical reduction in stability of around $10 \%$.

Modeling Unequally Distributed Ice Accretion It is the case that airplanes will not accumulate ice evenly between the wings and control surfaces. It is of interest to model a scenario where only the horizontal tail has developed any ice accretion. For this scenario, rather than applying (3.21) directly to a parameter, each parameter can be split into two components and then added together using the component buildup method. The component buildup method is essentially breaking up a parameter into a sum of the contributions to the parameter from each component of the airplane. This can only be achieved if the relative distribution of lift and drag between the wing fuselage and horizontal tail is known. With the parameter split into two components it is then possible to apply separate icing factors to the lift and drag contributions of the wing and horizontal tail [3] [4]. 
It is assumed that differing levels of ice accumulation appear as changes in the lift force and drag force on the respective aircraft component effected by ice. It is shown in [3] and [4] that the horizontal tail produces approximately $20 \%$ of the lift that the wing and fuselage generate. A similar result was reached for drag as the horizontal tail drag was approximately $20 \%$ of the drag created by the wings. Based on these results a $20 \%$ proportion was chosen for the distribution of lift and drag between the wing and fuselage and horizontal tail [3] [5]. It is important to note that this relationship only applies to the Cessna 208 Super Cargomaster. Other aircraft would need to be analyzed and conclusions drawn pertaining to the correlation between the wings, fuselage, and horizontal tail. In equation form, this proportion is simply represented as

$$
C_{L_{H}}=0.2 C_{L_{W F}}, C_{D_{0_{H}}}=0.2 C_{D_{0_{W}}}
$$

where $C_{L_{H}}$ is the contribution to the lift portion of a parameter from the horizontal tail, $C_{L_{W F}}$ is the contribution to the lift portion of a parameter from the wings and fuselage, $C_{D_{0_{H}}}$ is the contribution to the drag portion of a parameter from the horizontal tail at a predefined initial condition, and $C_{D_{0_{W}}}$ is the contribution to the drag portion of a parameter due to the wings at a predefined initial condition.

For example, consider the case of $Z_{\alpha}^{\prime}$. Extending the idea used to arrive at equations (3.24) and (3.25) as well as the information obtained from [4] and [5], $Z_{\alpha}^{\prime}$ can be expressed as

$$
Z_{\alpha}^{\prime}=Z_{L_{\alpha}}^{\prime}+Z_{D_{1}}^{\prime}
$$


Using the component buildup method (3.30) becomes

$$
Z_{\alpha}^{\prime}=Z_{L_{\alpha_{H}}}^{\prime}+Z_{L_{\alpha_{W F}}}^{\prime}+Z_{D_{1_{H}}}^{\prime}+Z_{D_{1_{W}}}^{\prime}
$$

where $Z_{L_{\alpha_{H}}}^{\prime}$ is the contribution to the lift portion of $Z_{\alpha}^{\prime}$ from the horizontal tail with respect to the angle of attack, $Z_{L_{\alpha_{W F}}}^{\prime}$ is the contribution to the lift portion of $Z_{\alpha}^{\prime}$ from the wings and fuselage with respect to the angle of attack, $Z_{D_{1_{H}}}^{\prime}$ is the contribution to the drag portion of $Z_{\alpha}^{\prime}$ from the horizontal tail at the steady state or equilibrium condition, and $Z_{D_{1_{W}}}^{\prime}$ is the contribution to the drag portion of $Z_{\alpha}^{\prime}$ from the wings at the steady state or equilibrium condition.

To calculate a parameter's value with horizontal tail icing only using the above information, the following steps can be followed.

Step 1 Using equation (3.31), the clean value for $Z_{\alpha}^{\prime}$ found in (3.11) at the flight conditions specified, and the proportion between lift and drag for the horizontal tail defined in (3.29) we can perform the following calculation to determine the value of the lift and drag portion of $Z_{\alpha}^{\prime}$ due solely to the wings.

$$
\begin{aligned}
Z_{\alpha}^{\prime} & =0.2 Z_{L_{\alpha_{W F}}}^{\prime}+Z_{L_{\alpha_{W F}}}^{\prime}+0.2 Z_{D_{1_{W}}}^{\prime}+Z_{D_{1_{W}}}^{\prime} \\
Z_{\alpha}^{\prime} & =1.2\left(Z_{L_{\alpha_{W F}}}^{\prime}+Z_{D_{1_{W}}}^{\prime}\right) \\
-1.17 & =1.2\left(Z_{L_{\alpha_{W F}}^{\prime}}^{\prime}+Z_{D_{1_{W}}}^{\prime}\right) \\
-0.975 & =\left(Z_{L_{\alpha_{W F}}}^{\prime}+Z_{D_{1_{W}}}^{\prime}\right)
\end{aligned}
$$

Step 2 Likewise, the following calculation can be performed to determine the numerical 
value of the lift and drag portion of $Z_{\alpha}^{\prime}$ due solely to the horizontal tail.

$$
\begin{aligned}
Z_{\alpha}^{\prime} & =5 Z_{L_{\alpha_{H}}}^{\prime}+Z_{L_{\alpha_{H}}}^{\prime}+5 Z_{D_{1_{H}}}^{\prime}+Z_{D_{1_{H}}}^{\prime} \\
Z_{\alpha}^{\prime} & =6\left(Z_{L_{\alpha_{H}}}^{\prime}+Z_{D_{1_{H}}}^{\prime}\right) \\
-1.17 & =6\left(Z_{L_{\alpha_{H}}}^{\prime}+Z_{D_{1_{H}}}^{\prime}\right) \\
-0.195 & =\left(Z_{L_{\alpha_{H}}}^{\prime}+Z_{D_{1_{H}}}^{\prime}\right)
\end{aligned}
$$

Step 3 Using the results of equations (3.32) and (3.33), as well as the value of $\Delta Z_{\alpha}$ from table 3.1 , the value of $Z_{\alpha_{i c e d}}^{\prime}$ with horizontal tail icing only can be calculated as,

$$
\begin{aligned}
& Z_{\alpha_{\text {iced }}}^{\prime}=\left(1+\Delta Z_{\alpha}\right)\left(Z_{L_{\alpha_{H}}}^{\prime}+Z_{D_{1_{H}}}^{\prime}\right)+\left(Z_{L_{\alpha_{W F}}}^{\prime}+Z_{D_{1_{W}}}^{\prime}\right) \\
& Z_{\alpha_{\text {iced }}}^{\prime}=(1+(-0.1))(-0.195)+(-0.975) \\
& Z_{\alpha_{\text {iced }}}^{\prime}=-1.1505
\end{aligned}
$$

In an attempt to generalize the above procedure, the process described above can be represented as

$$
Z_{\alpha_{\text {iced }}}^{\prime}=\left(1+\Delta Z_{\alpha}\right)\left(\frac{Z_{\alpha}^{\prime}}{6}\right)+\left(\frac{Z_{\alpha}^{\prime}}{1.2}\right)
$$

This process can be repeated for all the other parameters effected by icing to obtain a model that accounts for horizontal tail icing only. 


\subsubsection{Equations of Motion with Icing}

As stated previously, due to limtations on the information presented in [3] and [4] numerical values are not available for the equations of motion. However, using the procedures described in section 3.3.1 a general form for the equations of motion with ice accretion can be determined.

\section{Longitudinal Dynamic Model}

Worst Case Scenario Icing Physically, a worst case scenario icing condition would be when ice has developed on all vulnerable surfaces of the aircraft. In this research, this scenario is modeled by using the full value of $f_{i c e}$ and applying it to every parameter effected by icing. The parameters effected and the corresponding values for $f_{\text {ice }}$ are listed in table 3.1. The general form of the longitudinal equations of motion in a worst case scenario icing condition are presented below as,

$$
\begin{aligned}
\dot{u}= & -g \theta \cos \Theta_{1}+\left(X_{T_{u}}+X_{u}\right) u+X_{\alpha} \alpha+X_{q} q \\
& +X_{\delta_{e}} \delta_{e}+X_{\delta_{T}} \delta_{T}+X_{\dot{\alpha}} \dot{\alpha} \\
\dot{\alpha}= & \frac{\dot{\omega}}{U_{1}}=\left(-g \theta \sin \Theta_{1}+Z_{u} u+\left(1+\Delta Z_{\alpha}\right) Z_{\alpha} \alpha+\left(1+\Delta Z_{q}\right) Z_{q} q\right. \\
& \left.+U_{1} q+\left(1+\Delta Z_{\delta_{e}}\right) Z_{\delta_{e}} \delta_{e}+Z_{\delta_{T}} \delta_{T}+Z_{\dot{\alpha}} \dot{\alpha}\right) / U_{1} \\
\dot{q}= & \left(M_{T_{u}}+M_{u}\right) u+\left(M_{T_{\alpha}}+\left(1+\Delta M_{\alpha}\right) M_{\alpha}\right) \alpha+\left(1+\Delta M_{q}\right) M_{q} q \\
& +\left(1+\Delta M_{\delta_{e}}\right) M_{\delta_{e}} \delta_{e}+M_{\delta_{T}} \delta_{T}+M_{\dot{\alpha}} \dot{\alpha} \\
\dot{\theta}= & q .
\end{aligned}
$$


Worst Case Scenario Horizontal Tail Icing Physically, a worst case scenario horizontal tail icing condition would be when ice has developed only on the surfaces of the horizontal tail of the aircraft. In this research, this scenario is modeled by using the full value of $f_{i c e}$ and applying it to only the part of the parameter representing the lift and drag of the horizontal tail. The parameters effected and the corresponding values for $f_{\text {ice }}$ are listed in table 3.1. The general form of the longitudinal equations of motion in a worst case scenario horizontal tail icing condition are presented below as,

$$
\begin{aligned}
\dot{u}= & -g \theta \cos \Theta_{1}+\left(X_{T_{u}}+X_{u}\right) u+X_{\alpha} \alpha+X_{q} q \\
& +X_{\delta_{e}} \delta_{e}+X_{\delta_{T}} \delta_{T}+X_{\dot{\alpha}} \dot{\alpha} \\
\dot{\alpha}= & \frac{\dot{\omega}}{U_{1}}=\left(-g \theta \sin \Theta_{1}+Z_{u} u+\left(\left(1+\Delta Z_{\alpha}\right)\left(\frac{Z_{\alpha}}{6}\right)+\left(\frac{Z_{\alpha}}{1.2}\right)\right) \alpha\right. \\
& +\left(\left(1+\Delta Z_{q}\right)\left(\frac{Z_{q}}{6}\right)+\left(\frac{Z_{q}}{1.2}\right)\right) q+U_{1} q \\
& \left.+\left(\left(1+\Delta Z_{\delta_{e}}\right)\left(\frac{Z_{\delta_{e}}}{6}\right)+\left(\frac{Z_{\delta_{e}}}{1.2}\right)\right) \delta_{e}+Z_{\delta_{T}} \delta_{T}+Z_{\dot{\alpha}} \dot{\alpha}\right) / U_{1} \\
\dot{q}= & \left(M_{T_{u}}+M_{u}\right) u+\left(M_{T_{\alpha}}+\left(\left(1+\Delta M_{\alpha}\right)\left(\frac{M_{\alpha}}{6}\right)+\left(\frac{M_{\alpha}}{1.2}\right)\right)\right) \alpha \\
& +\left(\left(1+\Delta M_{q}\right)\left(\frac{M_{q}}{6}\right)+\left(\frac{M_{q}}{1.2}\right)\right) q \\
& +\left(\left(1+\Delta M_{\delta_{e}}\right)\left(\frac{M_{\delta_{e}}}{6}\right)+\left(\frac{M_{\delta_{e}}}{1.2}\right)\right) \delta_{e}+M_{\delta_{T}} \delta_{T}+M_{\dot{\alpha}} \dot{\alpha} \\
\dot{\theta}= & q
\end{aligned}
$$




\section{Lateral-Directional Dynamic Model}

Worst Case Scenario Icing Physically, a worst case scenario icing condition would be when ice has developed on all vulnerable surfaces of the aircraft. In this research, this scenario is modeled by using the full value of $f_{i c e}$ and applying it to every parameter effected by icing. The parameters effected and the corresponding values for $f_{\text {ice }}$ are listed in table 3.1. The general form of the lateral-directional equations of motion in a worst case scenario icing condition are presented below as,

$$
\begin{aligned}
\dot{p}= & \left(1+\Delta L_{\beta}\right) L_{\beta} \beta+\left(1+\Delta L_{p}\right) L_{p} p+L_{r} r+\left(1+\Delta L_{a}\right) L_{\delta_{a}} \delta_{a} \\
& +\left(1+\Delta L_{\delta_{R}}\right) L_{\delta_{R}} \delta_{R}+\frac{I_{x z}}{I_{x x}} \dot{r} \\
\dot{\phi}= & p+r \tan \Theta_{1} \\
\dot{\beta}= & \left(Y_{p} p+g \phi \cos \Theta_{1}+\left(1+\Delta Y_{\beta}\right) Y_{\beta} \beta+\left(Y_{r}-U_{1}\right) r\right. \\
& \left.+Y_{\delta_{a}} \delta_{a}+\left(1+\Delta Y_{\delta_{R}}\right) Y_{\delta_{R}} \delta_{R}\right) / U_{1} \\
\dot{r}= & \left(1+\Delta N_{\beta}\right) N_{\beta} \beta+N_{p} p+\left(1+\Delta N_{r}\right) N_{r} r+\left(1+\Delta N_{\delta_{a}}\right) N_{\delta_{a}} \delta_{a} \\
& +N_{\delta_{R}} \delta_{R}+\frac{I_{x z}}{I_{z z}} \dot{p} \\
\dot{\psi}= & r \sec \Theta_{1} .
\end{aligned}
$$

Worst Case Scenario Horizontal Tail Icing Physically, a worst case scenario horizontal tail icing condition would be when ice has developed only on the surfaces of the horizontal tail of the aircraft. In this research, this scenario is modeled by using the full value of $f_{i c e}$ and applying it to only the part of the parameter representing the lift and drag of the horizontal tail. The parameters effected and the 
corresponding values for $f_{\text {ice }}$ are listed in table 3.1. The general form of the lateraldirectional equations of motion in a worst case scenario horizontal tail icing condition are presented below as,

$$
\begin{aligned}
\dot{p}= & \left(\left(1+\Delta L_{\beta}\right)\left(\frac{L_{\beta}}{6}\right)+\left(\frac{L_{\beta}}{1.2}\right)\right) \beta+\left(\left(1+\Delta L_{p}\right)\left(\frac{L_{p}}{6}\right)+\left(\frac{L_{p}}{1.2}\right)\right) p \\
& +L_{r} r+\left(\left(1+\Delta L_{\delta_{a}}\right)\left(\frac{L_{\delta_{a}}}{6}\right)+\left(\frac{L_{\delta_{a}}}{1.2}\right)\right) \delta_{a} \\
& +\left(\left(1+\Delta L_{\delta_{R}}\right)\left(\frac{L_{\delta_{R}}}{6}\right)+\left(\frac{L_{\delta_{R}}}{1.2}\right)\right) \delta_{R}+\frac{I_{x z}}{I_{x x}} \dot{r} \\
\dot{\phi}= & p+r \tan \Theta_{1} \\
\dot{\beta}= & \left(Y_{p} p+g \phi \cos \Theta_{1}+\left(\left(1+\Delta Y_{\beta}\right)\left(\frac{Y_{\beta}}{6}\right)+\left(\frac{Y_{\beta}}{1.2}\right)\right) \beta+\left(Y_{r}-U_{1}\right) r\right. \\
& \left.+Y_{\delta_{a}} \delta_{a}+\left(\left(1+\Delta Y_{\delta_{R}}\right)\left(\frac{Y_{\delta_{R}}}{6}\right)+\left(\frac{Y_{\delta_{R}}}{1.2}\right)\right) \delta_{R}\right) / U_{1} \\
\dot{r}= & \left(\left(1+\Delta N_{\beta}\right)\left(\frac{N_{\beta}}{6}\right)+\left(\frac{N_{\beta}}{1.2}\right)\right) \beta+N_{p} p \\
& +\left(\left(1+\Delta N_{\delta_{r}}\right)\left(\frac{N_{\delta_{r}}}{6}\right)+\left(\frac{N_{\delta_{r}}}{1.2}\right)\right) r \\
& +\left(\left(1+\Delta N_{\delta_{a}}\right)\left(\frac{N_{\delta_{a}}}{6}\right)+\left(\frac{N_{\delta_{a}}}{1.2}\right)\right) \delta_{a}+N_{\delta_{R}} \delta_{R}+\frac{I_{x z}}{I_{z z}} \dot{p} \\
\dot{\psi}= & r \sec \Theta_{1} .
\end{aligned}
$$

\subsubsection{State Space Models with Icing}

With information obtained from [3] and [4], numerical values will be available for the state space models. Using the procedures described in section 3.3.1 a general form for the state space models with ice accretion will be presented along with numerical values. 


\section{Longitudinal Dynamic Model}

Worst Case Scenario Icing Physically, a worst case scenario icing condition would be when ice has developed on all vulnerable surfaces of the aircraft. In this research, this scenario is modeled by using the full value of $f_{i c e}$ and applying it to every parameter effected by icing. The parameters effected and the corresponding values for $f_{i c e}$ are listed in table 3.1. The general form of the system matrix $A$ and input matrix $B$ for the longitudinal dynamic model in a worst case scenario icing condition are presented below as,

$$
\begin{gathered}
A=\left[\begin{array}{cccc}
X_{u}^{\prime} & X_{\alpha}^{\prime} & X_{q}^{\prime} & X_{\theta}^{\prime} \\
Z_{u}^{\prime} & \left(1+\Delta Z_{\alpha}\right) Z_{\alpha}^{\prime} & \left(1+\Delta Z_{q}\right) Z_{q}^{\prime} & Z_{\theta}^{\prime} \\
M_{u}^{\prime} & \left(1+\Delta M_{\alpha}\right) M_{\alpha}^{\prime} & \left(1+\Delta M_{q}\right) M_{q}^{\prime} & M_{\theta}^{\prime} \\
0 & 0 & 1 & 0
\end{array}\right] \\
B=\left[\begin{array}{cc}
X_{\delta_{e}}^{\prime} & X_{\delta_{T}}^{\prime} \\
\left(1+\Delta Z_{\delta_{e}}\right) Z_{\delta_{e}}^{\prime} & Z_{\delta_{T}}^{\prime} \\
\left(1+\Delta M_{\delta_{e}}\right) M_{\delta_{e}}^{\prime} & M_{\delta_{T}}^{\prime} \\
0 & 0
\end{array}\right] .
\end{gathered}
$$

Using the information in (3.11) and the procedure defined in section 3.3.1 the numerical values for the longitudinal dynamic model in a worst case scenario icing condition can be calculated to be, 


$$
\left[\begin{array}{c}
\dot{u} \\
\dot{\alpha} \\
\dot{q} \\
\dot{\theta}
\end{array}\right]=\left[\begin{array}{cccc}
-0.03 & 17.74 & 0 & -32.17 \\
-0.0013 & -1.053 & 0.9584 & -0.0031 \\
-0.0005 & -7.8477 & -1.7273 & 0.0003 \\
0 & 0 & 1 & 0
\end{array}\right]\left[\begin{array}{l}
u \\
\alpha \\
q \\
\theta
\end{array}\right]+\left[\begin{array}{cc}
-0.55 & 2.79 \\
-0.0996 & 0 \\
-7.902 & 0 \\
0 & 0
\end{array}\right]\left[\begin{array}{c}
\delta_{e} \\
\delta_{T}
\end{array}\right] .
$$

Worst Case Scenario Horizontal Tail Icing Physically, a worst case scenario horizontal tail icing condition would be when ice has developed only on the surfaces of the horizontal tail of the aircraft. In this research, this scenario is modeled by using the full value of $f_{i c e}$ and applying it to only the part of the parameter representing the lift and drag of the horizontal tail. The parameters effected and the corresponding values for $f_{i c e}$ are listed in table 3.1. The general form of the system matrix $A$ and input matrix $B$ for the longitudinal dynamic model in a worst case scenario horizontal tail icing condition are presented below as,

$$
A=\left[\begin{array}{cccc}
X_{u}^{\prime} & X_{\alpha}^{\prime} & X_{q}^{\prime} & X_{\theta}^{\prime} \\
Z_{u}^{\prime} & \left(\left(1+\Delta Z_{\alpha}\right)\left(\frac{Z_{\alpha}^{\prime}}{6}\right)+\left(\frac{Z_{\alpha}^{\prime}}{1.2}\right)\right) & \left(\left(1+\Delta Z_{q}\right)\left(\frac{Z_{q}^{\prime}}{6}\right)+\left(\frac{Z_{q}^{\prime}}{1.2}\right)\right) & Z_{\theta}^{\prime} \\
M_{u}^{\prime} & \left(\left(1+\Delta M_{\alpha}\right)\left(\frac{M_{\alpha}^{\prime}}{6}\right)+\left(\frac{M_{\alpha}^{\prime}}{1.2}\right)\right) & \left(\left(1+\Delta M_{q}\right)\left(\frac{M_{q}^{\prime}}{6}\right)+\left(\frac{M_{q}^{\prime}}{1.2}\right)\right) & M_{\theta}^{\prime} \\
0 & 0 & 1 & 0
\end{array}\right],
$$




$$
B=\left[\begin{array}{cc}
X_{\delta_{e}}^{\prime} & X_{\delta_{T}}^{\prime} \\
\left(\left(1+\Delta Z_{\delta_{e}}\right)\left(\frac{Z_{\delta_{e}}^{\prime}}{6}\right)+\left(\frac{Z_{\delta_{e}}^{\prime}}{1.2}\right)\right) & Z_{\delta_{T}}^{\prime} \\
\left(\left(1+\Delta M_{\delta_{e}}\right)\left(\frac{M_{\delta_{e}}^{\prime}}{6}\right)+\left(\frac{M_{\delta_{e}}^{\prime}}{1.2}\right)\right) & M_{\delta_{T}}^{\prime} \\
0 & 0
\end{array}\right]
$$

Using the information in (3.11) and the procedure defined in section 3.3.1 the numerical values for the longitudinal dynamic model in a worst case scenario horizontal tail icing condition can be calculated to be,

$$
\left[\begin{array}{c}
\dot{u} \\
\dot{\alpha} \\
\dot{q} \\
\dot{\theta}
\end{array}\right]=\left[\begin{array}{cccc}
-0.03 & 17.74 & 0 & -32.17 \\
-0.0013 & -1.1505 & 0.9681 & -0.0031 \\
-0.0005 & -8.5663 & -1.7796 & 0.0003 \\
0 & 0 & 1 & 0
\end{array}\right]\left[\begin{array}{c}
u \\
\alpha \\
q \\
\theta
\end{array}\right]+\left[\begin{array}{cc}
-0.55 & 2.79 \\
-0.1083 & 0 \\
-8.6337 & 0 \\
0 & 0
\end{array}\right]\left[\begin{array}{c}
\delta_{e} \\
\delta_{T}
\end{array}\right] .
$$

\section{Lateral-Directional Dynamic Model}

Worst Case Scenario Icing Physically, a worst case scenario icing condition would be when ice has developed on all vulnerable surfaces of the aircraft. In this research, this scenario is modeled by using the full value of $f_{i c e}$ and applying it to every parameter effected by icing. The parameters effected and the corresponding values for $f_{\text {ice }}$ are listed in table 3.1. The general form of the system matrix $A$ and input matrix $B$ for the lateral-directional dynamic model in a worst case scenario icing condition are presented below as, 
$A=\left[\begin{array}{ccccr}\left(1+\Delta L_{p}\right) L_{p}^{\prime} & 0 & \left(1+\Delta L_{\beta}\right) L_{\beta}^{\prime} & L_{r}^{\prime} & 0 \\ 1 & 0 & 0 & \tan \Theta_{1} & 0 \\ \frac{Y_{p}}{U_{1}} & \frac{g \cos \Theta_{1}}{U_{1}} & \frac{\left(1+\Delta Y_{\beta}\right) Y_{\beta}}{U_{1}} & \left(\frac{Y_{r}}{U_{1}}-1\right) & 0 \\ N_{p}^{\prime} & 0 & \left(1+\Delta N_{\beta}\right) N_{\beta}^{\prime} & \left(1+\Delta N_{r}\right) N_{r}^{\prime} & 0 \\ 0 & 0 & 0 & \sec \Theta_{1} & 0\end{array}\right]$

$$
B=\left[\begin{array}{cc}
\left(1+\Delta L_{\delta_{a}}\right) L_{\delta_{a}}^{\prime} & \left(1+\Delta L_{\delta_{R}}\right) L_{\delta_{R}}^{\prime} \\
0 & 0 \\
\frac{Y_{\delta_{a}}}{U_{1}} & \frac{\left(1+\Delta Y_{\delta_{R}}\right) Y_{\delta_{R}}}{U_{1}} \\
\left(1+\Delta N_{\delta_{a}}\right) N_{\delta_{a}}^{\prime} & N_{\delta_{R}}^{\prime} \\
0 & 0
\end{array}\right]
$$

Using the information in (3.19) and the procedure defined in section 3.3.1 the numerical values for the lateral-directional dynamic model in a worst case scenario icing condition can be calculated to be, 


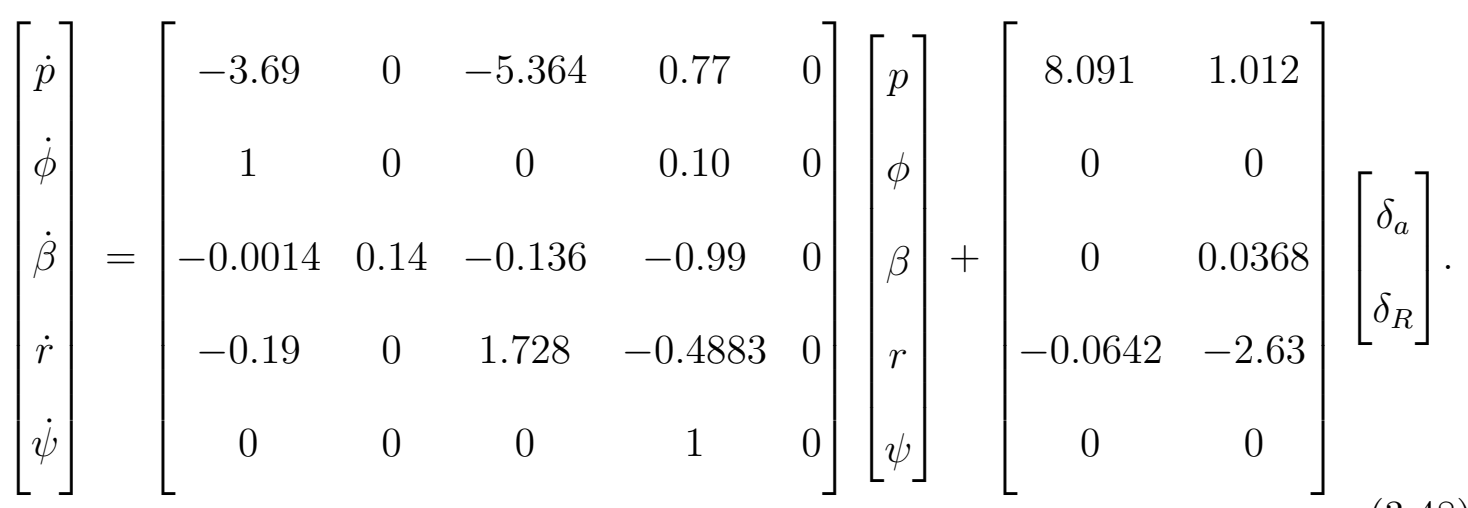

Worst Case Scenario Horizontal Tail Icing Physically, a worst case scenario horizontal tail icing condition would be when ice has developed only on the surfaces of the horizontal tail of the aircraft. In this research, this scenario is modeled by using the full value of $f_{i c e}$ and applying it to only the part of the parameter representing the lift and drag of the horizontal tail. The parameters effected and the corresponding values for $f_{\text {ice }}$ are listed in table 3.1. The general form of the system matrix $A$ and input matrix $B$ for the lateral-directional dynamic model in a worst case scenario horizontal tail icing condition are presented below as, 
$A=\left[\begin{array}{ccccc}\left(\left(1+\Delta L_{p}\right)\left(\frac{L_{p}^{\prime}}{6}\right)+\left(\frac{L_{p}^{\prime}}{1.2}\right)\right) & 0 & \left(\left(1+\Delta L_{\beta}\right)\left(\frac{L_{\beta}^{\prime}}{6}\right)+\left(\frac{L_{\beta}^{\prime}}{1.2}\right)\right) & L_{r}^{\prime} & 0 \\ 1 & 0 & 0 & \tan \Theta_{1} & 0 \\ \frac{Y_{p}}{U_{1}} & \frac{g \cos \Theta_{1}}{U_{1}} & \frac{\left(\left(1+\Delta Y_{\beta}\right)\left(\frac{Y_{\beta}^{\prime}}{6}\right)+\left(\frac{Y_{\beta}^{\prime}}{1.2}\right)\right)}{U_{1}} & \left(\frac{Y_{r}}{U_{1}}-1\right) & 0 \\ N_{p}^{\prime} & 0 & \left(\left(1+\Delta N_{\beta}\right)\left(\frac{N_{\beta}^{\prime}}{6}\right)+\left(\frac{N_{\beta}^{\prime}}{1.2}\right)\right) & \left(\left(1+\Delta N_{\delta_{r}}\right)\left(\frac{N_{\delta_{r}}^{\prime}}{6}\right)+\left(\frac{N_{\delta_{r}}^{\prime}}{1.2}\right)\right) & 0 \\ 0 & 0 & 0 & \sec \Theta_{1} & 0\end{array}\right]$,

$$
B=\left[\begin{array}{cc}
\left(\left(1+\Delta L_{\delta_{a}}\right)\left(\frac{L_{\delta_{a}}^{\prime}}{6}\right)+\left(\frac{L_{\delta_{a}}^{\prime}}{1.2}\right)\right) & \left(\left(1+\Delta L_{\delta_{R}}\right)\left(\frac{L_{\delta_{R}}^{\prime}}{6}\right)+\left(\frac{L_{\delta_{R}}^{\prime}}{1.2}\right)\right) \\
0 & 0 \\
\frac{\left(\left(1+\Delta Y_{\delta_{R}}\right)\left(\frac{Y_{\delta_{a}}^{\prime}}{6}\right)+\left(\frac{Y_{\delta_{R}}^{\prime}}{1.2}\right)\right)}{U_{1}} \\
\left(\left(1+\Delta N_{\delta_{a}}\right)\left(\frac{N_{\delta_{a}}^{\prime}}{6}\right)+\left(\frac{N_{\delta_{a}}^{\prime}}{1.2}\right)\right) \\
0
\end{array}\right]
$$

Using the information in (3.19) and the procedure defined in section 3.3.1 the numerical values for the lateral-directional dynamic model in a worst case scenario horizontal tail icing condition can be calculated to be, 


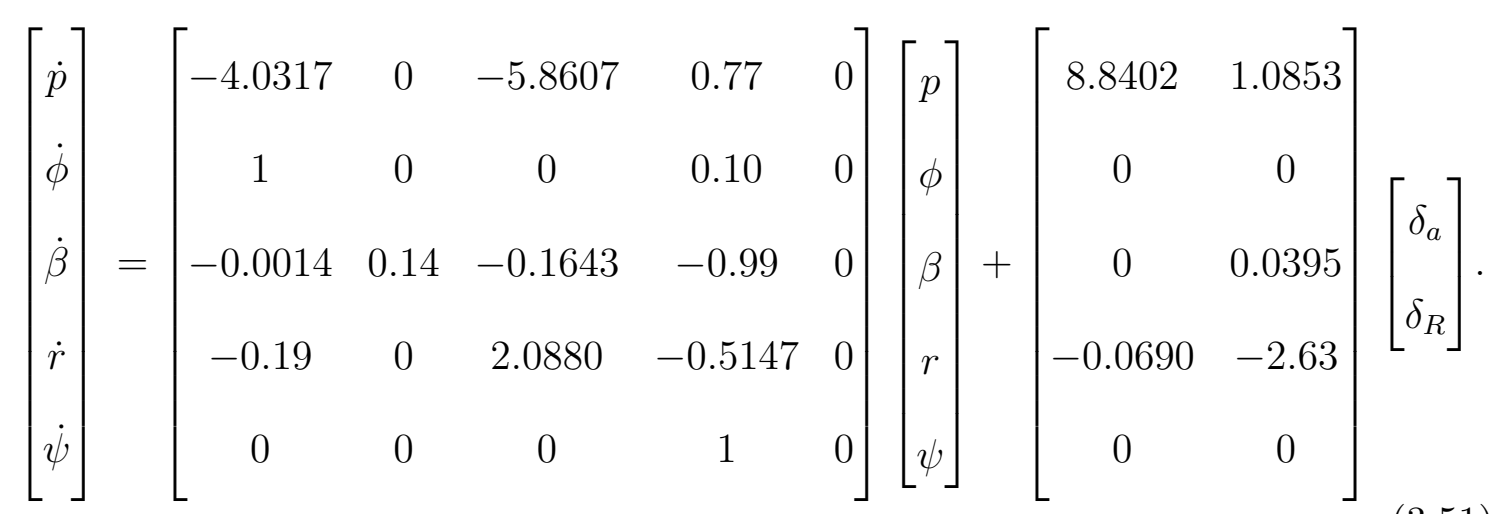

\subsection{Summary}

In this chapter the equations of motion and state space models for the longitudinal and lateral-directional dynamics were presented. Following that discussion, an icing effects model applicable to any individual performance, stability, or control parameter or derivative effected by icing was discussed. This icing effects model was then expanded upon so that it would apply to a worst case scenario icing condition and a horizontal tail icing condition. It was then used to model a worst case scenario icing condition and a worst case scenario horizontal tail icing condition for both the longitudinal and lateral-directional dynamics. Numerical values for the clean, worst case scenario icing, and worst case scenario horizontal tail icing longitudinal and lateral-directional dynamics were presented. 


\section{Chapter 4}

\section{Adaptive Control Design}

The design of an adaptive control system will make use of the background information presented in chapter 2. In this work, state feedback will be utilized. It can be used as part of an adaptive control algorithm for either state tracking or output tracking. Stability properties as well as performance objectives are important to consider in the design process and will be examined in detail forthcoming.

\subsection{Control Objectives and Design Conditions}

The design of either an adaptive control system for state tracking or output tracking is performed by keeping two control objectives in mind. The first objective is to ensure that all signals present in the closed loop system are bounded. The second objective is to ensure that asymptotic tracking of some desired signal or vector signal is achieved. The signal or vector signal to be tracked varies depending on the adaptive control system's configuration. The design conditions that must be met 
for adaptive control design differs largely on the choice between state tracking and output tracking. More detail regarding these control objectives and design conditions will be provided in the following sections.

\subsubsection{Limitations of State Tracking Design}

For state tracking, a reference state vector, $x_{m}(t)$, is generated and asymptotically tracked by the changing system state vector, $x_{p}(t)$. The reference state vector, $x_{m}(t)$, is generated from the reference system

$$
\dot{x}_{m}(t)=A_{m} x_{m}(t)+b_{m} r(t)
$$

where the reference input is $r(t) \in \mathbb{R}$, the reference system matrix $A_{m} \in \mathbb{R}^{n \times n}$ is known and constant, the reference input matrix $b_{m} \in \mathbb{R}^{n}$ is known and constant, and the intial reference state is $x_{m}(0)=x_{m 0}$.

In this thesis, the matrix $A_{m}$ and $b_{m}$ are the system and input matrices for the longitudinal and lateral-directional state space models in no icing conditions, respectively. It is important to note that the system input matrix $b_{m}$ corresponds to only one of the inputs available in either the longitudinal or lateral-directional state space models. For example, if the elevator deflection angle, $\delta_{e}$, is to be considered as the input, $b_{m}$ would consist of only the first column of the input matrix, $B$, of (3.7). Values for the $A_{m}$ and $b_{m}$ matrices can be found in section 3.2.2.

In order for the control objectives to be met a number of design conditions must be true. It is assumed that

(A4.1) All the eigenvalues of $A_{m}$ are in the open left half complex plane; 
(A4.2) $r(t)$ is bounded and piecewise continuous;

(A4.3) There exists a constant vector $k_{1}^{*} \in \mathbb{R}^{n}$ and a non-zero constant scalar $k_{2}^{*} \in \mathbb{R}$ such that the following equations are satisfied:

$$
A_{p}+b_{p} k_{1}^{* T}=A_{m}, \quad b_{p} k_{2}^{*}=b_{m}
$$

where $A_{p}$ and $b_{p}$ are the longitudinal and lateral-directional system and input matrices, respectively, in either a worst case scenario icing or worst case scenario horizontal tail icing condition;

(A4.4) $\operatorname{sign}\left[k_{2}^{*}\right]$, the sign of the parameter $k_{2}^{*}$ is known [20].

If any of the above design conditions are not true then it is not possible to obtain an adaptive control design.

The design conditions A4.1 and A4.2 are to ensure that the reference system will be stable, well-defined, and produce a bounded output $y_{m}(t)$. A quick check of the state space models of section 3.2.2 guarantees that design condition A4.1 is valid. For this research, $r(t)$ is chosen to be a step input of 1 therefore guaranteeing that design condition A4.2 is valid. When performing the calculations of the matching condition (4.2), detailed in $\mathbf{A 4 . 3}$, it is found that no solution for $k_{1}^{*}$ exists for any of the models.

Since $k_{1}^{*}$ does not exist, an adaptive control design for state tracking is not possible. There is no way for an ideal controller to exist that can make $A_{p}$ to be $A_{m}$. If an ideal controller does not exist for state tracking then an adaptive controller will not exist because there are no possible values for $k_{1}$ and $k_{2}$ for the system to calculate 
with this configuration. The matching condition (4.2) is a major limitation of state tracking due to the difficulty encountered when attempting to satisfy the matching equations.

\subsubsection{Matching Conditions for Output Tracking}

As in section 4.1.1 there are design conditions that must be met in order to ensure the control design objective of closed loop signal boundedness and asymptotic tracking of a reference output signal, $y_{m}(t)$, by the actual system output $y(t)$. Before arriving at these design conditions, first consider a linear time-invariant state space model of the form

$$
\dot{x}(t)=A x(t)+B u(t), y(t)=C x(t),
$$

where $x(t) \in \mathbb{R}^{n}, u(t) \in \mathbb{R}$, and $y(t) \in \mathbb{R}$. The matrices $A \in \mathbb{R}^{n \times n}, B \in \mathbb{R}^{n \times 1}$, and $C \in \mathbb{R}^{1 \times n}$ with $n>0$ are unknown and constant. The transfer function of the system is

$$
y(s)=C(s I-A)^{-1} B u(s)=\frac{Z(s)}{P(s)} u(s),
$$

where

$$
Z(s)=z_{m} s^{m}+\cdots+z_{1} s+z_{0}
$$

with $Z(s)$ being a monic polynomial of degree $n$ and $z_{m} \neq 0$.

In this thesis, the matrices $A$ and $B$ can be the matrices defined in the longitudinal and lateral-directional state space models for a worst case scenario icing and worst 
case scenario horizontal tail icing condition, respectively. It is important to note again that the system input matrix, $B$, corresponds to only one of the inputs available. The explanation is identical to the explanation for $b_{m}$ in section 4.1.1. These matrices can be found in section 3.3.3.

As mentioned previously, in order that the control objectives be met a number of design conditions must be valid. It is assumed that

(A4.5) $(A, B, C)$ is stabilizable and detectable;

(A4.6) $Z(s)$ is a stable polynomial;

(A4.7) The degree $m$ of $Z(s)$ is known;

(A4.8) The sign of $z_{m}, \operatorname{sign}\left(z_{m}\right)$, is known.

The design condition A4.5 is needed for output matching and internal signal boundedness [20]. Design condition A4.6 is needed so that $y(t)$ will track a given $y_{m}(t)$. This also implies that $(A, B, C)$ is stabilizable and detectable. Design condition A4.7 is needed for determining a reference model. Finally, design condition A4.8 is needed so that an adaptive parameter update law may be designed [20].

With equation (4.4) and the assumptions above, an attempt can be made at choosing reference models for the respective systems of the longitudinal and lateraldirectional dynamic models. The reference model for each system is chosen to be of the form

$$
y_{m}(s)=W_{m}(s) r(s), W_{m}(s)=\frac{1}{P_{m}(s)},
$$

where $P_{m}(s)$ is the desired closed loop characteristic polynomial of degree $n-m$. 
With $P_{m}(s)$ chosen, it is necessary to guarantee that ideal gains $K^{*}$ and $k_{r}^{*}$ exist for the same reasons as those given in section 4.1.1. If they exist, then it is possible to calculate them from the matching equation

$$
\operatorname{det}\left(s I-A-B K^{*}\right)=P_{m}(s) Z(s) \frac{1}{z_{m}}, \quad k_{r}^{*}=\frac{1}{z_{m}} .
$$

\subsection{Output Tracking Control Design}

In this section design conditions will be verified for both the longitudinal and lateral-directional models in worst case scenario and worst case scenario horizontal tail icing conditions. Transfer functions and ideal gains for each situation are presented. Details regarding an adaptive controller structure for an output tracking scheme are given as well as a discussion on it's stability properties.

\subsubsection{Design Condition Verification}

The design conditions discussed in section 4.1 .2 will now be verified for each transfer function that is able to be obtained from the longitudinal and lateral-directional state space models. The worst case scenario icing and worst case scenario horizontal tail icing longitudinal models will be checked followed by the lateral-directional models.

\section{Longitudinal Model Design Condition Verification}

Worst Case Scenario Icing Using (4.4) and the matrices $A$ and $B$ obtained from section 3.3.3 for the longitudinal state space model in a worst case scenario 
icing encounter, the transfer functions for the inputs $\delta_{e}$ and $\delta_{T}$ to each respective output can be obtained. The transfer functions are presented in table 4.1.

Each transfer function can be analyzed with the design conditions A4.5, A4.6, A4.7, and A4.8 to ensure whether or not an adaptive control system design can be achieved for each output, respectively. After comparison, the result is that only two transfer functions for the longitudinal model in a worst case scenario icing encounter satisfy all design condtions. The two transfer functions are those that correspond to the input $\delta_{e}$ to the outputs $\alpha$ and $\theta$, respectively. The ideal gains associated with these two transfer functions are calculated using (4.7). The ideal gains for the input $\delta_{e}$ to the output $\alpha$ are

$$
\begin{aligned}
K^{*} & =\left[\begin{array}{llll}
-0.0130575 & -0.532389 & 9.62668 & -0.0311812
\end{array}\right]^{T}, \\
k_{r}^{*} & =-10.0452 .
\end{aligned}
$$

Likewise, the ideal gains for the input $\delta_{e}$ to the output $\theta$ are

$$
\begin{aligned}
K^{*} & =\left[\begin{array}{llll}
-0.0000632956 & -0.993123 & -0.0920526 & 0.126583
\end{array}\right]^{T}, \\
k_{r}^{*} & =-0.12655
\end{aligned}
$$

Worst Case Scenario Horizontal Tail Icing Using (4.4) and the matrices A and $B$ obtained from section 3.3.3 for the longitudinal state space model in a worst case scenario horizontal tail icing encounter, the transfer functions for the inputs 
Table 4.1: Transfer functions for the worst case scenario icing longitudinal state space model.

\begin{tabular}{lc}
\hline \hline From input $\delta_{e}$ to output $\ldots$ \\
\hline$u:$ & $\frac{-0.55 s^{3}-3.295 s^{2}+111.7 s+243}{s^{4}+2.81 s^{3}+9.446 s^{2}+0.2878 s+0.3105}$ \\
$\alpha:$ & $\frac{-0.09955 s^{3}-7.747 s^{2}-0.2063 s-0.3281}{s^{4}+2.81 s^{3}+9.446 s^{2}+0.2878 s+0.3105-0.10}$ \\
$q:$ & $\frac{-7.902 s^{3}-7.776 s^{2}-0.4129 s}{s^{4}+2.81 s^{3}+9.446 s^{2}+0.2878 s+0.3105}$ \\
$\theta:$ & $\frac{-7.902 s^{2}-7.776 s-0.4129}{s^{4}+2.81 s^{3}+9.446 s^{2}+0.2878 s+0.3105}$ \\
\hline From input $\delta_{T}$ to output $\ldots$ \\
\hline$u:$ & $\frac{2.79 s^{3}+7.757 s^{2}+26.06 s-0.06876}{s^{4}+2.81 s^{3}+9.446 s^{2}+0.2878 s+0.3105}$ \\
$\alpha:$ & $\frac{-0.003627 s^{2}-0.007602 s+0.000005413}{s^{4}+2.81 s^{3}+9.446 s^{2}+0.2878 s+0.3105}$ \\
$q:$ & $\frac{-0.001395 s^{2}+0.02699 s}{s^{4}+2.81 s^{3}+9.446 s^{2}+0.2878 s+0.3105}$ \\
$\theta:$ & $\frac{-0.001395 s+0.02699}{s^{4}+2.81 s^{3}+9.446 s^{2}+0.2878 s+0.3105}$ \\
\hline \hline
\end{tabular}

$\delta_{e}$ and $\delta_{T}$ to each respective output can be obtained. The transfer functions are presented in table 4.2 .

After comparison with A4.5, A4.6, A4.7, and A4.8, the result is that two transfer functions for the longitudinal model in a worst case scenario horizontal tail icing encounter satisfy all design condtions. Again, the two transfer functions are those that correspond to the input $\delta_{e}$ to the outputs $\alpha$ and $\theta$, respectively. The ideal gains associated with these two transfer functions are calculated using (4.7). The ideal gains for the input $\delta_{e}$ to the output $\alpha$ are 


$$
\begin{aligned}
K^{*} & =\left[\begin{array}{llll}
-0.0120029 & -1.39002 & 8.93851 & -0.0286146
\end{array}\right]^{T}, \\
k_{r}^{*} & =-9.23361 .
\end{aligned}
$$

Likewise, the ideal gains for the input $\delta_{e}$ to the output $\theta$ are

$$
\begin{aligned}
K^{*} & =\left[\begin{array}{llll}
-0.0000578944 & -0.992183 & -0.090303 & 0.115851
\end{array}\right]^{T}, \\
k_{r}^{*} & =-0.115821
\end{aligned}
$$

Table 4.2: Transfer functions for the worst case scenario horizontal tail icing longitudinal state space model.

\begin{tabular}{cc}
\hline \hline From input $\delta_{e}$ to output $\ldots$ \\
\hline$u:$ & $\frac{-0.55 s^{3}-3.532 s^{2}+120.4 s+290.2}{s^{4}+2.96 s^{3}+10.45 s^{2}+0.3168 s+0.3389}$ \\
$\alpha:$ & $\frac{-0.1083 s^{3}-8.553 s^{2}-0.2282 s-0.3585}{s^{4}+2.96 s^{3}+10.45 s^{2}+0.3168 s+0.3389}$ \\
$q:$ & $\frac{-8.634 s^{3}-9.264 s^{2}-0.4741 s}{s^{4}+2.96 s^{3}+10.45 s^{2}+0.3168 s+0.3389}$ \\
$\theta:$ & $\frac{-8.634 s^{2}-9.264 s-0.4741}{s^{4}+2.96 s^{3}+10.45 s^{2}+0.3168 s+0.3389}$ \\
\hline From input $\delta_{T}$ to output $\ldots$ \\
\hline$u:$ & $\frac{2.79 s^{3}+8.175 s^{2}+28.85 s-0.07505}{s^{4}+2.96 s^{3}+10.45 s^{2}+0.3168 s+0.3389}$ \\
$\alpha:$ & $\frac{-0.003627 s^{2}-0.007805 s+0.000005413}{s^{4}+2.96 s^{3}+10.45 s^{2}+0.3168 s+0.3389}$ \\
$q:$ & $\frac{-0.001395 s^{2}+0.02946 s}{s^{4}+2.96 s^{3}+10.45 s^{2}+0.3168 s+0.3389}$ \\
$\theta:$ & $\frac{-0.001395 s+0.02946}{s^{4}+2.96 s^{3}+10.45 s^{2}+0.3168 s+0.3389}$ \\
\hline \hline
\end{tabular}




\section{Lateral-Directional Model Design Condition Verification}

Worst Case Scenario Icing Using (4.4) and the matrices $A$ and $B$ obtained from section 3.3.3 for the lateral-directional state space model in a worst case scenario icing encounter, the transfer functions for the inputs $\delta_{a}$ and $\delta_{R}$ to each respective output can be obtained. The transfer functions are presented in table 4.3.

After comparison with A4.5, A4.6, A4.7, and A4.8, the result is that there are four transfer functions for the lateral-directional model in a worst case scenario icing encounter that satisfy all design condtions. The transfer functions are those that correspond to the input $\delta_{a}$ to the outputs $\phi$ and $\beta$ as well as to the input $\delta_{R}$ to the outputs $r$ and $\psi$, respectively.

A problem arises when calculating the ideal gains for the transfer function with an input originating from $\delta_{a}$ to the outputs $\phi$ and $\beta$ as well as the input originating from $\delta_{R}$ to the output $r$. A polynomial $P_{m}(s)$ can be chosen, but with it the resulting polynomials on either side of the equality in equation (4.7) are not of the same order. This means that the ideal gains cannot be calculated. This is due to the fact that one of the eigenvalues, or poles, of the system matrix, $A$, in (3.48) is equal to zero. Due to this, the system is either not stabilizable or not detectable though it appears that it would be when performing tests to ensure A4.5 is valid.

Due to the inability of design condition A4.5 to be satisfied, only one adaptive control system is possible. The ideal gains associated with the transfer function with input $\delta_{R}$ to the output $\psi$ are calculated using (4.7). The ideal gains are 


$$
\begin{aligned}
K^{*} & =\left[\begin{array}{lllll}
-0.0715973 & -0.0000156716 & 0.657837 & 0.194674 & 0.380184
\end{array}\right]^{T}, \\
k_{r}^{*} & =-0.380228 .
\end{aligned}
$$

Table 4.3: Transfer functions for the worst case scenario icing lateral-directional state space model.

\begin{tabular}{lc}
\hline \hline From input $\delta_{a}$ to output $\ldots$ \\
\hline$p:$ & $\frac{8.091 s^{3}+5.002 s^{2}+14.03 s-0.1909}{s^{4}+4.314 s^{3}+4.22 s^{2}+8.311 s+0.07686}$ \\
$\phi:$ & $\frac{8.085 s^{2}+4.823 s+14.01}{s^{4}+4.314 s^{3}+4.22 s^{2}+8.311 s+0.07686}$ \\
$\beta:$ & $\frac{0.05222 s^{2}+2.883 s+0.5213}{s^{4}+4.314 s^{3}+4.22 s^{2}+8.311 s+0.07686}$ \\
$r:$ & $\frac{-0.06419 s^{3}-1.783 s^{2}-0.2604 s+1.909}{s^{4}+4.314 s^{3}+4.22 s^{2}+8.311 s+0.07686}$ \\
$\psi:$ & $\frac{-0.06419 s^{3}-1.783 s^{2}-0.2604 s+1.909}{s^{5}+4.314 s^{4}+4.22 s^{3}+8.311 s^{2}+0.07686 s}$ \\
\hline From $\operatorname{input~} \delta_{R}$ to output $\ldots$ \\
\hline$p:$ & $\frac{1.012 s^{3}-1.591 s^{2}-12.49 s+0.173}{s^{4}+4.314 s^{3}+4.22 s^{2}+8.311 s+0.07686}$ \\
$\phi:$ & $\frac{0.749 s^{2}-2.61 s-12.6}{s^{4}+4.314 s^{3}+4.22 s^{2}+8.311 s+0.07686}$ \\
$\beta:$ & $\frac{0.0368 s^{3}+2.756 s^{2}+9.977 s-0.3529}{s^{4}+4.314 s^{3}+4.22 s^{2}+8.311 s+0.07686}$ \\
$r:$ & $\frac{-2.63 s^{3}-10.19 s^{2}-1.057 s-1.73}{s^{4}+4.314 s^{3}+4.22 s^{2}+8.311 s+0.07686}$ \\
$\psi:$ & $\frac{-2.63 s^{3}-10.19 s^{2}-1.057 s-1.73}{s^{5}+4.314 s^{4}+4.22 s^{3}+8.311 s^{2}+0.07686 s}$ \\
\hline \hline
\end{tabular}

Worst Case Scenario Horizontal Tail Icing Using (4.4) and the matrices $A$ and $B$ obtained from section 3.3.3 for the lateral-directional state space model in 
a worst case scenario horizontal tail icing encounter, the transfer functions for the inputs $\delta_{a}$ and $\delta_{R}$ to each respective output can be obtained. The transfer functions are presented in table 4.4 .

After comparison with A4.5, A4.6, A4.7, and A4.8, the result is that there are four transfer functions for the lateral-directional model in a worst case scenario horizontal tail icing encounter that satisfy all design conditions. The transfer functions are of the same inputs and outputs as those of the lateral-directional model in a worst case scenario icing condition. They correspond to the input $\delta_{a}$ to the outputs $\phi$ and $\beta$ as well as to the input $\delta_{R}$ to the outputs $r$ and $\psi$, respectively.

Again, a problem arises when calculating the ideal gains for the transfer function with an input originating from $\delta_{a}$ to the outputs $\phi$ and $\beta$ as well as the input originating from $\delta_{R}$ to the output $r$. A polynomial $P_{m}(s)$ can be chosen, but with it the resulting polynomials on either side of the equality in equation (4.7) are not of the same order. This means that the ideal gains cannot be calculated for the very same reason as before. Due to this, the system is either not stabilizable or not detectable though it appears that it would be when performing tests to ensure $\mathbf{A 4 . 5}$ is valid.

Due to the inability of design condition $\mathbf{A 4 . 5}$ to be satisfied, only one adaptive control system is possible. The ideal gains associated with the transfer function with input $\delta_{R}$ to the output $\psi$ are calculated using (4.7). The ideal gains are 


$$
\begin{aligned}
K^{*} & =\left[\begin{array}{lllll}
-0.539621 & 0.0284752 & 0.04554 & 0.146255 & 0.404578
\end{array}\right]^{T}, \\
k_{r}^{*} & =-0.380228
\end{aligned}
$$

Table 4.4: Transfer functions for the worst case scenario horizontal tail icing lateraldirectional state space model.

\begin{tabular}{lc}
\hline \hline From input $\delta_{a}$ to output $\ldots$ \\
\hline$p:$ & $\frac{8.84 s^{3}+5.95 s^{2}+18.61 s-0.2528}{s^{4}+4.711 s^{3}+5.027 s^{2}+10.59 s+0.06379}$ \\
$\phi:$ & $\frac{8.833 s^{2}+5.753 s+18.58}{s^{4}+4.711 s^{3}+5.027 s^{2}+10.59 s+0.06379}$ \\
$\beta:$ & $\frac{0.05597 s^{2}+3.169 s+0.6022}{s^{4}+4.711 s^{3}+5.027 s^{2}+10.59 s+0.06379}$ \\
$r:$ & $\frac{-0.06903 s^{3}-1.969 s^{2}-0.347 s+2.528}{s^{4}+4.711 s^{3}+5.027 s^{2}+10.59 s+0.06379}$ \\
$\psi:$ & $\frac{-0.06903 s^{3}-1.969 s^{2}-0.347 s+2.528}{s^{5}+4.711 s^{4}+5.027 s^{3}+10.59 s^{2}+0.06379 s}$ \\
\hline From $\operatorname{input~} \delta_{R}$ to output $\ldots$ \\
\hline$p:$ & $\frac{1.085 s^{3}-1.519 s^{2}-13.31 s+0.1841}{s^{4}+4.711 s^{3}+5.027 s^{2}+10.59 s+0.06379}$ \\
$\phi:$ & $\frac{0.8223 s^{2}-2.635 s-13.45}{s^{4}+4.711 s^{3}+5.027 s^{2}+10.59 s+0.06379}$ \\
$\beta:$ & $\frac{0.03947 s^{3}+2.782 s^{2}+10.91 s-0.3566}{s^{4}+4.711 s^{3}+5.027 s^{2}+10.59 s+0.06379}$ \\
$r:$ & $\frac{-2.63 s^{3}-11.16 s^{2}-1.382 s-1.841}{s^{4}+4.711 s^{3}+5.027 s^{2}+10.59 s+0.06379}$ \\
$\psi:$ & $\frac{-2.63 s^{3}-11.16 s^{2}-1.382 s-1.841}{s^{5}+4.711 s^{4}+5.027 s^{3}+10.59 s^{2}+0.06379 s}$ \\
\hline \hline
\end{tabular}




\subsubsection{Controller Structure}

With the ideal gains calculated and presented in section 4.2.1 for each scenario suitable for adaptive control, a fixed gain controller can be defined to achieve the desired control objective as

$$
u(t)=K^{* T} x(t)+k_{r}^{*} r(t) .
$$

This controller will produce the desired closed-loop system $y(s)=W_{m}(s) r(s)$. However, these ideal gains are unknown since $A, B$, and $C$ are unknown. A controller must be defined that uses estimates of the ideal gains. Using these estimates the model reference adaptive controller structure is

$$
u(t)=K^{T}(t) x(t)+k_{r}(t) u(t) .
$$

With the adaptive controller structure (4.21) the state space equations defined in (4.3) become

$$
\begin{aligned}
\dot{x}(t) & =A x(t)+B\left(K^{T}(t) x(t)+k_{r}(t) r(t)\right) \\
& =\left(A+B K^{* T}\right) x(t)+B k_{r}^{*} r(t)+B\left(\left(K^{T}(t)-K^{*}\right) x(t)+\left(k_{r}(t)-k_{r}^{*}\right) r(t)\right) \\
y(t) & =C x(t) .
\end{aligned}
$$

\subsubsection{Estimation Error}

Before an equation for the estimation error can be developed it is critical to have defined the tracking error for the systems. Using (4.6), (4.22), and the fact that 


$$
C\left(s I-A-B K^{* T}\right)^{-1} B k_{r}^{*}=\frac{Z(s) k_{r}^{*}}{\operatorname{det}\left(s I-A-B K^{* T}\right)}=\frac{1}{P_{m}(s)}=W_{m}(s)
$$

the tracking error is defined as

$$
\begin{aligned}
e(t) & =y(t)-y_{m}(t) \\
& =\rho^{*} W_{m}(s)\left[\left(K^{T}-K^{* T} x+\left(k_{r}-k_{r}^{*}\right) r\right](t)+C e^{\left(A+B K^{* T}\right) t} x(0),\right.
\end{aligned}
$$

where $\rho^{*}=z_{m}$ and $C e^{\left(A+B K^{* T}\right) t} x(0)$ decays to zero due to the stability of $A+B K^{* T}$ $[20]$.

With the tracking error defined it is also important to define some auxiliary signals. They will be helpful in defining the estimation error as well as the adaptive laws for the systems. These signals are defined to be

$$
\begin{aligned}
\theta(t) & =\left[\begin{array}{ll}
K^{T}(t) & k_{r}(t)
\end{array}\right]^{T}, \\
\theta^{*} & =\left[\begin{array}{ll}
K^{* T} & k_{r}^{*}
\end{array}\right]^{T}, \\
\omega(t) & =\left[\begin{array}{ll}
x^{T}(t) & r(t)
\end{array}\right]^{T}, \\
\zeta(t) & =W_{m}(s)[w](t), \\
\xi(t) & =\theta^{T}(t) \zeta(t)-W_{m}(s)\left[\theta^{T} \omega\right](t) .
\end{aligned}
$$

Finally, the estimation error can be defined as

$$
\epsilon(t)=e(t)+\rho(t) \xi(t),
$$


where $\rho(t)$ is the estimate of $\rho^{*}$. Substituting (4.24)-(4.29) into equation (4.30) and ignoring the $C e^{\left(A+B K^{* T}\right) t} x(0)$ term, we have

$$
\epsilon(t)=\rho^{*}\left(\theta(t)-\theta^{*}\right)^{T} \zeta(t)+\left(\rho(t)-\rho^{*}\right) \xi(t) .
$$

\subsubsection{Adaptive Laws}

To determine the adaptive laws needed to update the estimates $\theta(t)$ and $\rho(t)$ a procedure based on the normalized gradient algorithm will be used [20]. Just as in the normalized gradient algorithm, a quadratic cost function

$$
J(\theta, \rho)=\frac{\epsilon^{2}}{2 m^{2}(t)}
$$

will be considered, where $\mathrm{m}(\mathrm{t})$ is a normalizing signal that will be chosen later. It is desireable to update $\theta(t)$ and $\rho(t)$ along the steepest descent direction of (4.32). For $\theta(t)$, taking the partial derivative of (4.32) with respect to $\theta(t)$ gives

$$
\frac{\partial J}{\partial \theta}=-\frac{\rho^{*} \zeta(t) \epsilon(t)}{m^{2}(t)}
$$

with the adaptive law following to be

$$
\dot{\theta}(t)=-\frac{\operatorname{sign}\left[\rho^{*}\right] \Gamma \zeta(t) \epsilon(t)}{m^{2}(t)}, \theta\left(t_{0}\right)=\theta_{0},
$$

where $\Gamma=\Gamma^{T}>0 \in \mathbb{R}^{(n+1) \times(n+1)}$ is a constant matrix and $\theta_{0}$ is the initial estimate of $\theta^{*}$. Likewise, for $\rho(t)$, taking the partial derivative of (4.32) with respect to $\rho(t)$ gives 


$$
\frac{\partial J}{\partial \rho}=-\frac{\xi(t) \epsilon(t)}{m^{2}(t)}
$$

with the adaptive law following to be

$$
\dot{\rho}(t)=-\frac{\gamma \xi(t) \epsilon(t)}{m^{2}(t)}, \rho\left(t_{0}\right)=\rho_{0},
$$

where $\gamma>0$ is a constant and $\rho_{0}$ is the initial estimate of $\rho^{*}$. The matrix $\Gamma$ and the constant $\gamma$ are adaptation gains with their values being a design choice.

To choose the normalizing signal $m(t)$, the boundedness of $\frac{\epsilon(t)}{m(t)}$ must be ensured when $\theta(t)$ and $\rho(t)$ are bounded [20]. According to [20], this can be guaranteed by the choice

$$
m(t)=\sqrt{1+\zeta^{T}(t) \zeta(t)+\xi^{2}(t)}
$$

\subsection{Stability Properties}

The stability properties of an adaptive control algorithm are the measure of its adequacy. For output tracking, it is necessary that all signals in the closed-loop system be bounded. Physically, this is important to avoid damage that may occur from an unbounded signal present in the system.

To show that all desired properties can be obtained, Lyapunov stability theory will be employed. To begin, a positive definite function

$$
V(\tilde{\theta}, \tilde{\rho})=\frac{1}{2}\left(\left|\rho^{*}\right| \tilde{\theta}^{T} \Gamma^{-1} \tilde{\theta}+\gamma^{-1} \tilde{\rho}^{2}\right)
$$


is defined, where

$$
\tilde{\theta}(t)=\theta(t)-\theta^{*}, \quad \tilde{\rho}(t)=\rho(t)-\rho^{*} .
$$

Taking the derivative of (4.38) with respect to time and using (4.34) as well as (4.36) gives $\dot{V}(\tilde{\theta}, \tilde{\rho})$ to be

$$
\dot{V}=-\frac{\epsilon^{2}(t)}{m^{2}(t)}
$$

Since (4.40) is negative definite, the adaptive laws (4.34) and (4.36) ensure that $\theta(t) \in L^{\infty}, \rho(t) \in L^{\infty}, \frac{\epsilon(t)}{m(t)} \in L^{2} \cap L^{\infty}, \dot{\theta}(t) \in L^{2} \cap L^{\infty}$, and $\dot{\rho}(t) \in L^{2} \cap L^{\infty}[20]$.

With the above properties obtained from the lyapunov analysis, all signals in the closed-loop system are bounded. With this, the tracking error, $e(t)$, satisfies the following two properties

$$
\begin{aligned}
& \lim _{t \rightarrow \infty} y(t)-y_{m}(t)=0, \\
& \int_{0}^{\infty}\left(y(t)-y_{m}(t)\right)^{2} d t<\infty .
\end{aligned}
$$

For a proof of the above statements regarding the tracking error properties, (4.41) and (4.42), please see [20].

\subsection{Summary}

In this chapter adaptive control theory relevant to this research was presented. The control objectives and design conditions were stated along with a discussion about the limitations of the matching condition for state tracking. Due to this, state 
tracking was not a viable method for adaptive control and instead output tracking was employed. Only some of the inputs to each respective output of the longitudinal and lateral-directional state space models satisfied the relaxed design conditions of output tracking when compared to state tracking. Following that discussion, the adaptive controller structure was presented along with a formulation for the estimation error. The estimation error expression that is given is then used to develop adaptive laws for the gains of the controller. To conclude the chapter, a brief description of the stability properties was given with a reference that includes more detail regarding the proof of statements made in this concluding section. 


\section{Chapter 5}

\section{Adaptive Control System}

\section{Simulations}

For all of the following simulations, effects on the system dynamics due to icing begin to occur at 30 seconds and peak at 100 seconds. Those conditions at 100 seconds remain constant for the duration of the simulation and reflect the worst case scenario icing encounter for the longitudinal and lateral-directional models. Fixed gain controllers were simulated to compare their performance to that of the designed adaptive controllers. The fixed gain simulation results are presented immediately with the adaptive gain simulation results for easy comparison. A reference step input, $r(t)=1$, is used for each simulation. The adaptation gains for the longitudinal simulations were chosen to be

$$
\Gamma=10 I \text { and } \gamma=5,
$$

where $I \in \mathbb{R}^{(n+1) \times(n+1)}$ is the identity matrix and $n=4$. The adaptation gains for 
the lateral-directional simulations were chosen to be

$$
\Gamma=10 I \text { and } \gamma=5
$$

where $I \in \mathbb{R}^{(n+1) \times(n+1)}$ is the identity matrix and $n=5$. There will be a section immediately following the simulations for each scenario discussing the results.

\subsection{Worst Case Scenario Icing}

\subsubsection{Longitudinal Dynamic Model}

\section{Situation}

For this simulation, the aircraft is flying in atmospheric conditions that pose no possibility for ice accretion until the 30 second mark. At 30 seconds it has been modeled that the aircraft flies directly into atmospheric conditions perfectly suited to the accretion of ice. There is an initial instantaneous freezing of liquid water droplets followed by a constant increase in ice accretion on the vulnerable surfaces of the aircraft corresponding to the terms of the longitudinal dynamics. The phenomena of this instantaneous freeze is the study of supercooled icing theory. More information on this theory and the resultant effects on an aircraft can be found in [38], [50], [49], and [51]. This instant freeze does not amount to a great deal of ice accretion. However, the effects are noticed immediately in the simulations. The terms of the longitudinal dynamics that are effected by ice accretion are discussed in section 3.3.3. Beyond the 30 second mark ice continues to accumulate until the 
time reaches 100 seconds. At 100 seconds the ice accretion reaches a maximum.

\section{Simulation Results}

The outputs $\alpha$ and $\theta$, in a worst case scenario icing condition, are of interest for adaptive control. These outputs are dependent upon the input originating from $\delta_{e}$. The ideal gains for the respective controllers were calculated using the matching condition (4.7). The values for these ideal gains were presented in section 4.2 .1 and are reproduced here as

$$
\begin{aligned}
K^{*} & =\left[\begin{array}{llll}
-0.0130575 & -0.532389 & 9.62668 & -0.0311812
\end{array}\right]^{T}, \\
k_{r}^{*} & =-10.0452
\end{aligned}
$$

for $\alpha$ and

$$
\begin{aligned}
K^{*} & =\left[\begin{array}{llll}
-0.0000632956 & -0.993123 & -0.0920526 & 0.126583
\end{array}\right]^{T}, \\
k_{r}^{*} & =-0.12655 .
\end{aligned}
$$

for $\theta$. These two sets of $K^{*}$ and $k_{r}^{*}$ are the values that would make up the nominal controller,

$$
u(t)=K^{* T} x(t)+k_{r}^{*} r(t)
$$

that would control the outputs $\alpha$ and $\theta$ in a worst case scenario icing encounter, respectively. However, this nominal controller is unknown because the system and input matrices for a worst case scenario icing encounter are unknown. Therefore, the estimates $K(t)$ and $k_{r}(t)$ of $K^{*}$ and $k_{r}^{*}$ will be determined from adaptive laws and 
used in the adaptive controller structure

$$
u(t)=K^{T}(t) x(t)+k_{r}(t) u(t) .
$$

For the purposes of the simulation, the ideal gains for the longitudinal state space model in no icing conditions were calculated. They are used to show how a fixed gain controller cannot handle the changes in aerodynamics that result from ice accretion on an aircraft. The gains are calculated using (4.7) and are presented as

$$
\begin{aligned}
K^{*} & =\left[\begin{array}{llll}
-0.0118181 & -1.54545 & 8.8181 & -0.0281874
\end{array}\right]^{T}, \\
k_{r}^{*} & =-9.09091
\end{aligned}
$$

for $\alpha$ and

$$
\begin{aligned}
K^{*} & =\left[\begin{array}{llll}
-0.000056937 & -0.992037 & -0.0899721 & 0.113933
\end{array}\right]^{T}, \\
k_{r}^{*} & =-0.113895
\end{aligned}
$$

for $\theta$.

The simulations for the fixed gain controllers for the output tracking of $\alpha$ and $\theta$ are shown in figures 5.1 and 5.5, respectively. The adaptive simulations for $\alpha$ are presented in figures 5.2, 5.3, and 5.4. Likewise, the adaptive simulations for $\theta$ are presented in figures 5.6, 5.7, and 5.8. 


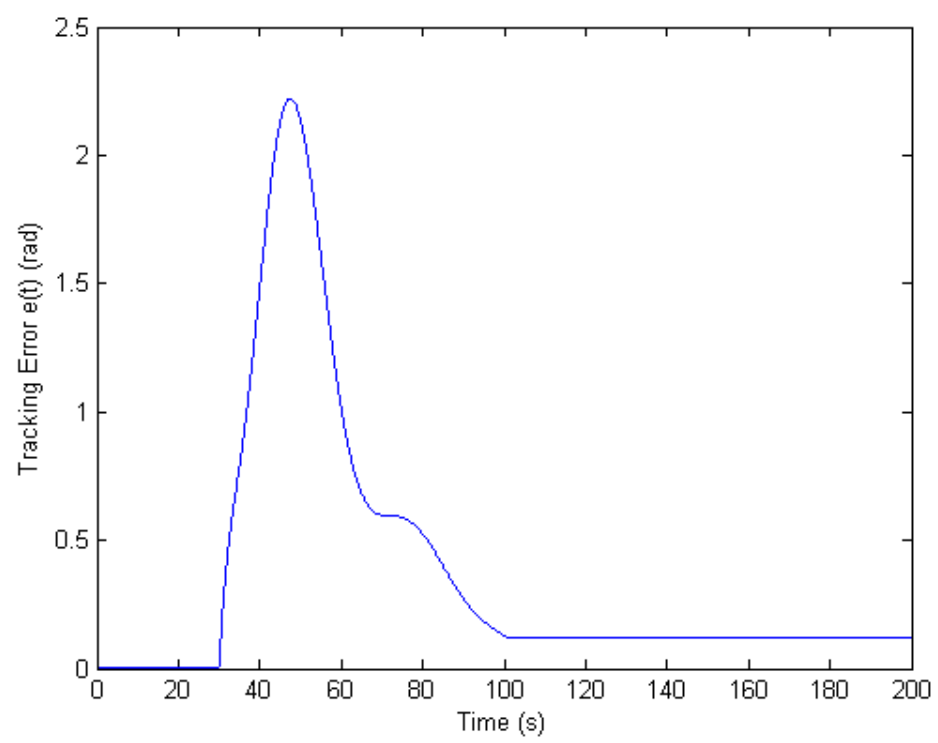

Figure 5.1: Output tracking error for the fixed gain control of the angle of attack, $\alpha$, in a worst case scenario icing encounter at $t=30 \mathrm{~s}$.

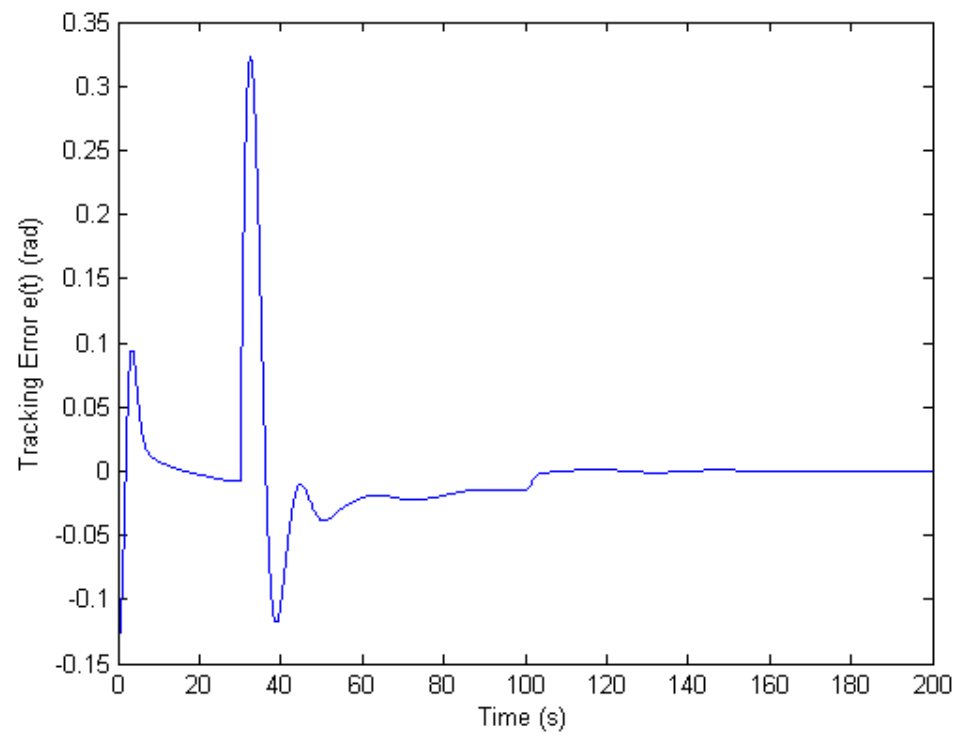

Figure 5.2: Output tracking error for the adaptive control of the angle of attack, $\alpha$, in a worst case scenario icing encounter at $t=30 \mathrm{~s}$. 

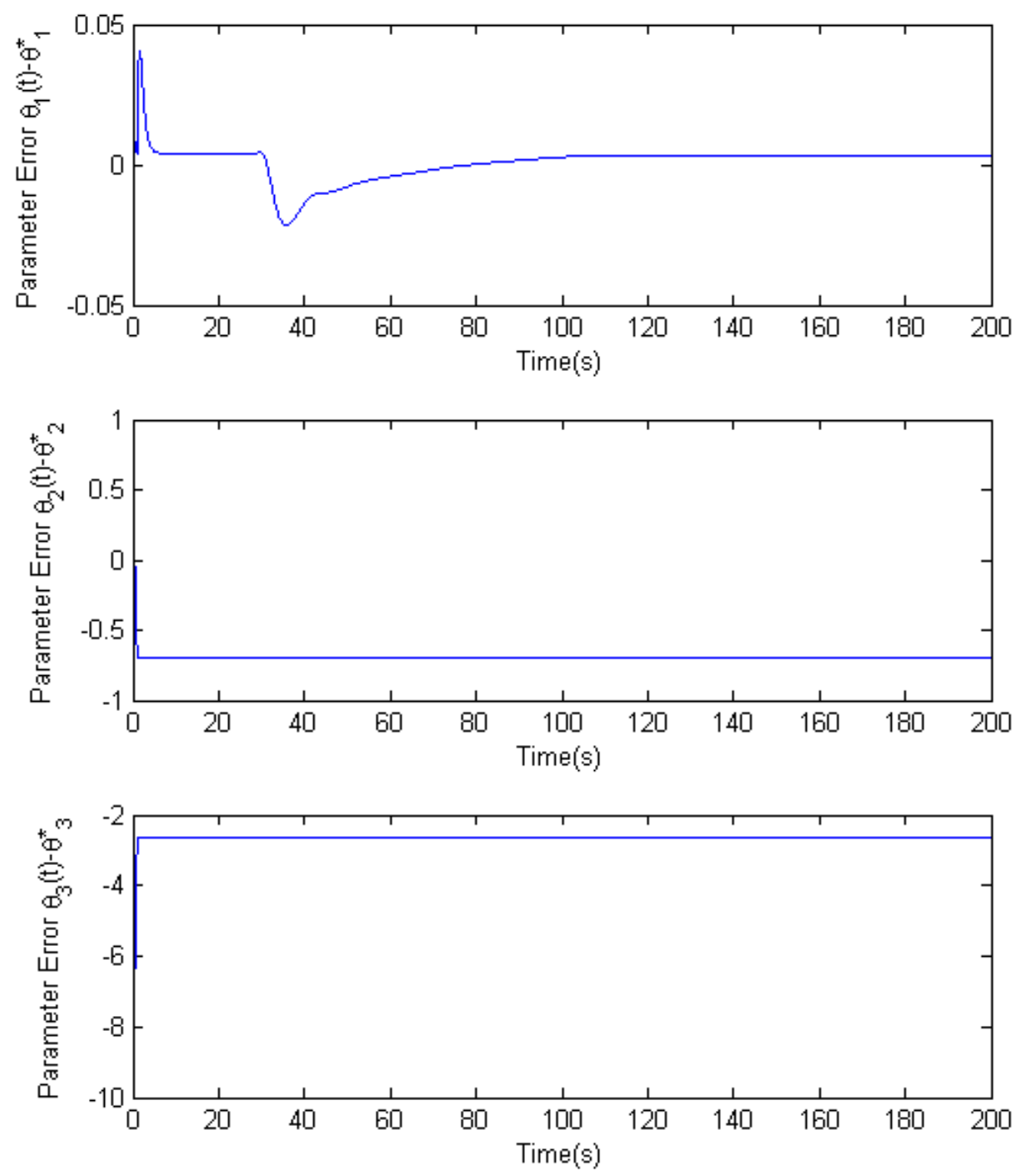

Figure 5.3: Parameter errors for $\theta_{1}, \theta_{2}$, and $\theta_{3}$ for the adaptive control of the angle of attack, $\alpha$, in a worst case scenario icing encounter at $t=30 \mathrm{~s}$. 

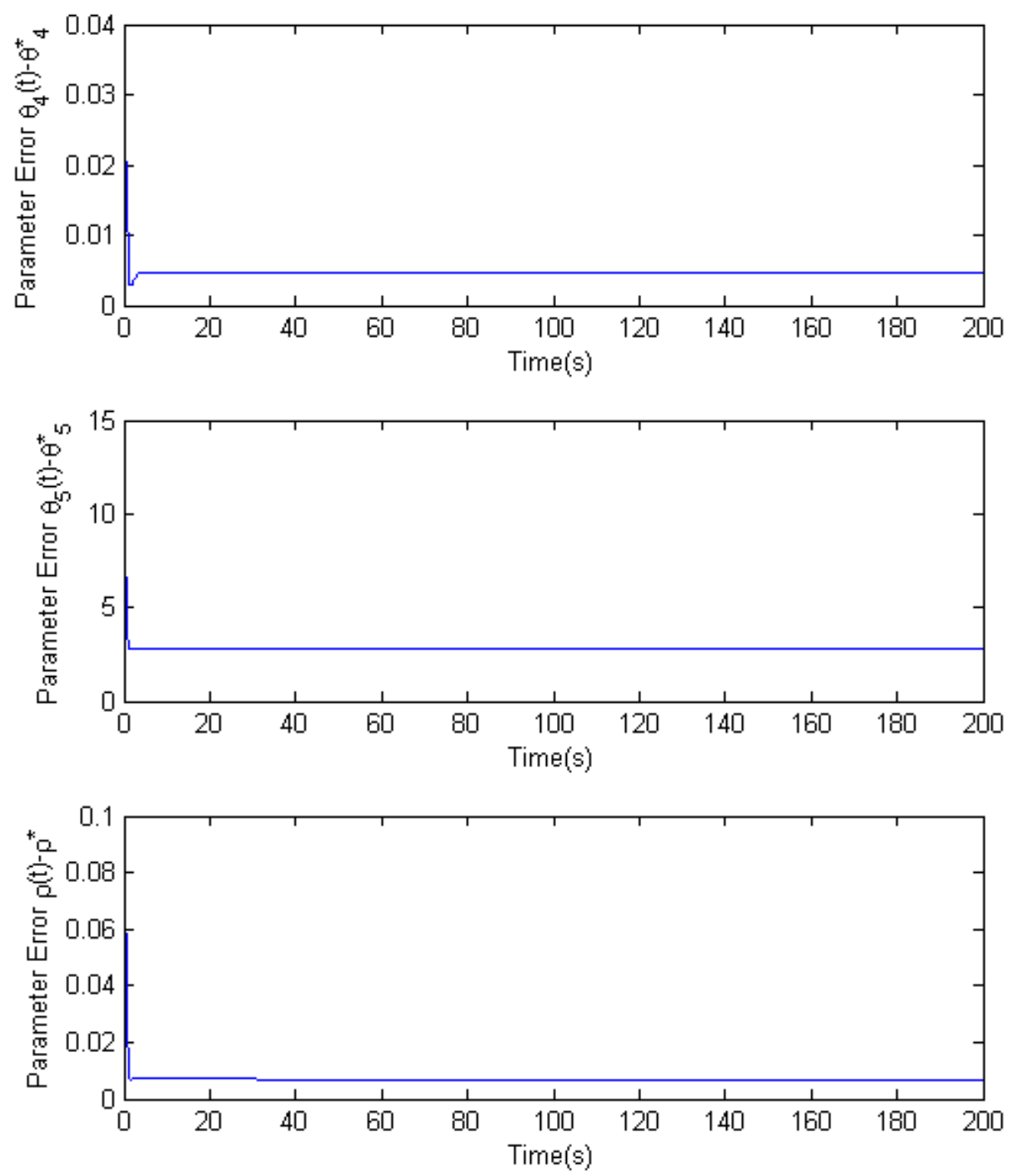

Figure 5.4: Parameter errors for $\theta_{4}, \theta_{5}$, and $\rho$ for the adaptive control of the angle of attack, $\alpha$, in a worst case scenario icing encounter at $t=30 \mathrm{~s}$. 


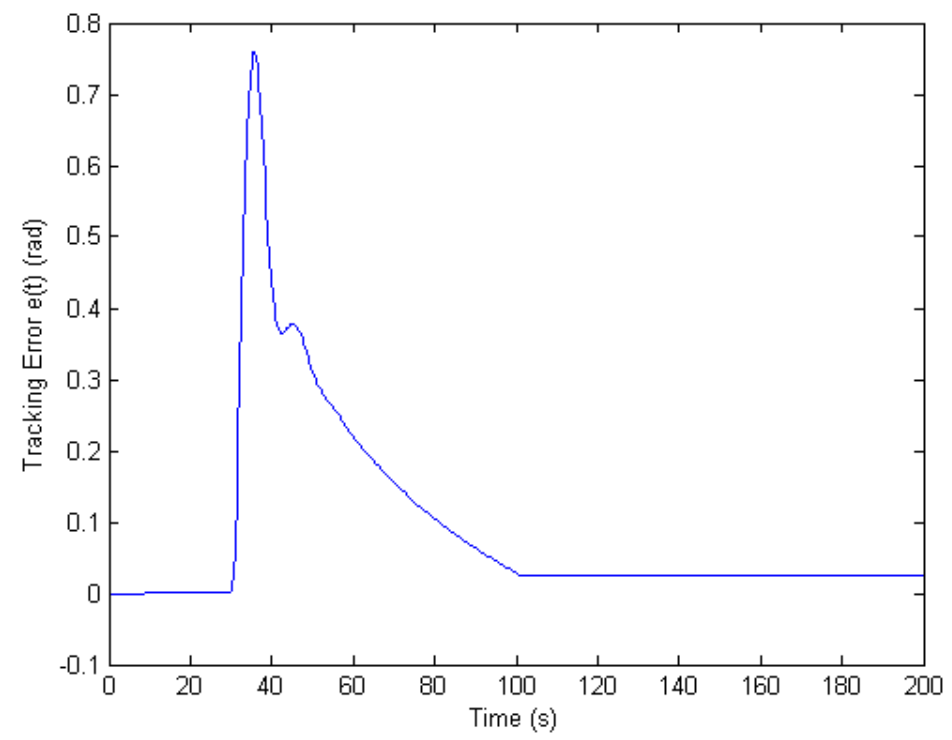

Figure 5.5: Output tracking error for the fixed gain control of the pitch attitude angle, $\theta$, in a worst case scenario icing encounter at $t=30 \mathrm{~s}$.

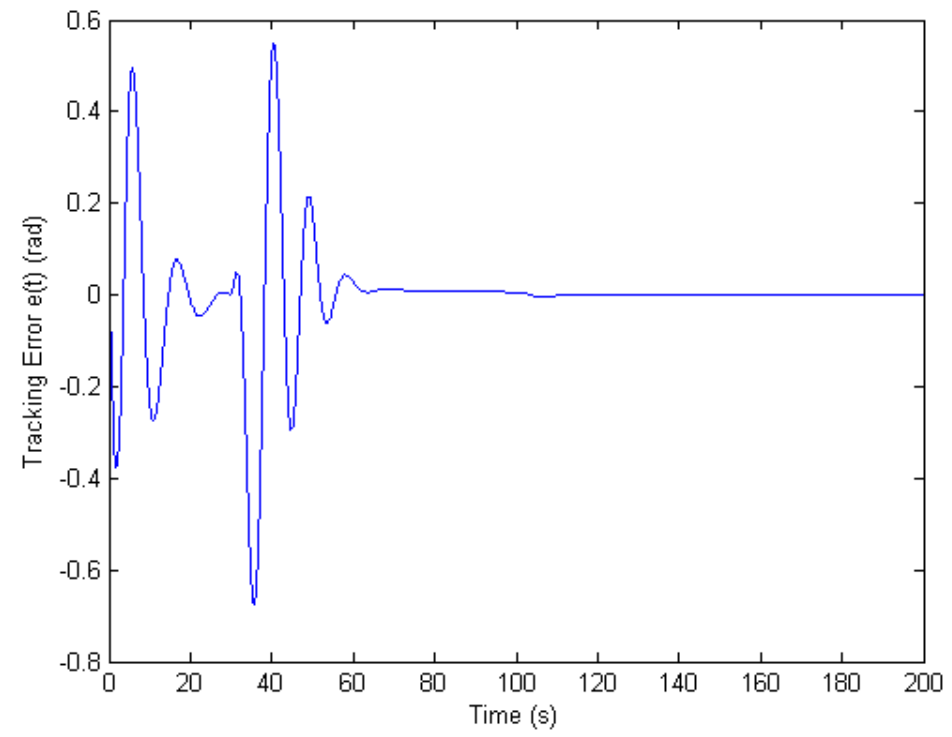

Figure 5.6: Output tracking error for the adaptive control of the pitch attitude angle, $\theta$, in a worst case scenario icing encounter at $t=30 \mathrm{~s}$. 

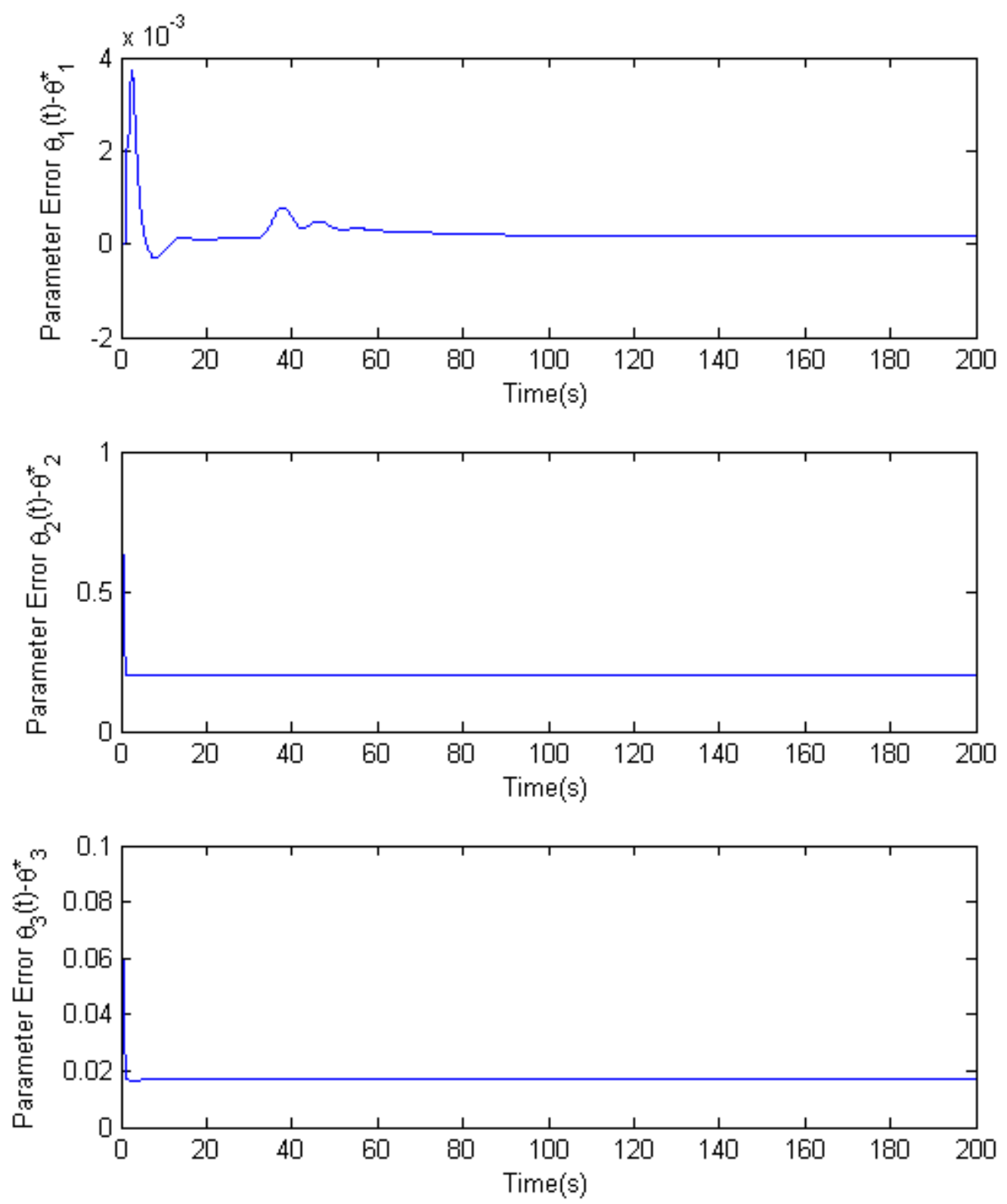

Figure 5.7: Parameter errors for $\theta_{1}, \theta_{2}$, and $\theta_{3}$ for the adaptive control of the pitch attitude angle, $\theta$, in a worst case scenario icing encounter at $t=30 \mathrm{~s}$. 

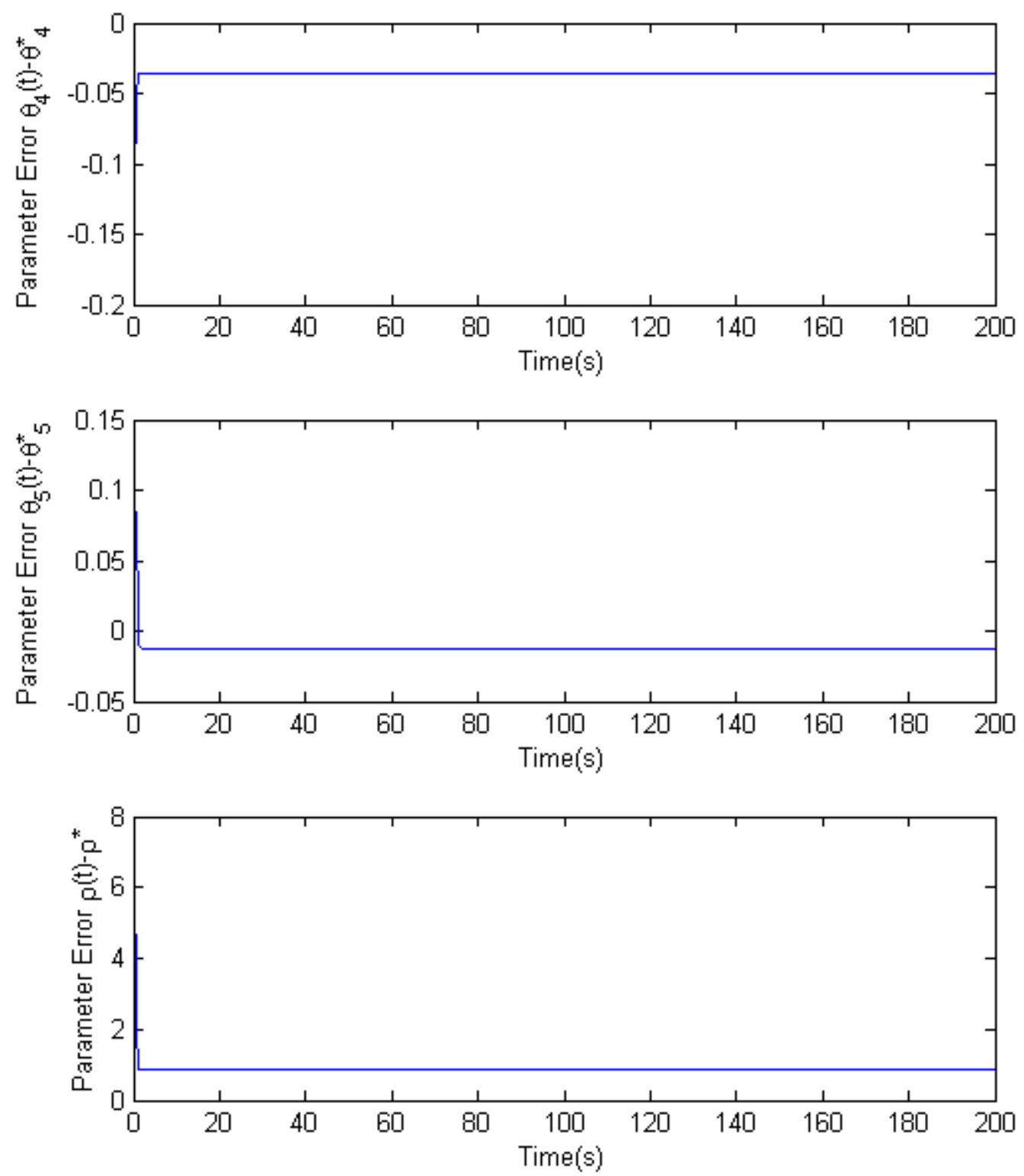

Figure 5.8: Parameter errors for $\theta_{4}, \theta_{5}$, and $\rho$ for the adaptive control of the pitch attitude angle, $\theta$, in a worst case scenario icing encounter at $t=30 \mathrm{~s}$. 


\section{Discussion}

Figures 5.1 and 5.5 clearly show that as soon as ice accumulates on the surfaces of an aircraft, a change in flight dynamics occurs. Notice, that the fixed gain controller is able to reduce the tracking error after about 100 seconds to a level that would appear to have a negligible affect on the ability of the autopilot to maintain steady state flight. However, while in flight if the autopilot were to experience an uncommanded change in either $\alpha$ or $\theta$ it would surely attempt to correct it. Such an attempt would only delay the decrease in tracking error. It is possible that if the autopilot continued to make attempts to correct the aircraft it may never return to the steady state flight condition. This would also be true if the autopilot automatically disconnected and the pilot was forced to take command of the plane manually. Overly aggressive commands by the pilot could result in loss of control.

Another observation that can be made about figures 5.1 and 5.5 is that even if the pilot commanded the equivalent of a step input throughout the change in flight dynamics due to ice accretion, the time it takes for the fixed gain controller to reduce the tracking error may be too long before the aircraft encounters a disastrous collision of some sort. Granted, this may not be a potential problem while in the air but during a descent to land this could be catastrophic.

The adaptive controller handles the change in flight dynamics well bringing the tracking error to zero for both the outputs $\alpha$ and $\theta$. It is easy to assume that since the adaptive controller takes just as long, or longer, than the classical controller to decrease the tracking error that the results are uninspiring. However, with the correct choice of the adaptation gains $\Gamma$ and $\gamma$ this time can be decreased dramatically. 
Notice that the parameter errors do not converge to zero. This is not guaranteed when using an adaptive controller. However, all parameters converge to a constant value relatively close to the ideal parameters. Parameter convergence is dependent on the richness of the system input signal. For more information on the factors governing parameter converge see [20]. 


\subsubsection{Lateral-Directional Dynamic Model}

\section{Situation}

For this simulation the situation is the same as that in section 5.1.1. However, now there is an initial instantaneous freezing of liquid water droplets followed by a constant increase in ice accretion on the vulnerable surfaces of the aircraft corresponding to the terms of the lateral-directional dynamics. The terms of the lateral-directional dynamics that are effected by ice accretion are discussed in section 3.3.3.

\section{Simulation Results}

The output $\psi$, in a worst case scenario icing condition, is of interest for adaptive control. This output is dependent upon the input originating from $\delta_{R}$. The ideal gains for this controller were calculated using the matching condition (4.7). The values for these ideal gains are presented in section 4.2.1 and are reproduced here as

$$
\begin{aligned}
K^{*} & =\left[\begin{array}{lllll}
-0.0715973 & -0.0000156716 & 0.657837 & 0.194674 & 0.380184
\end{array}\right]^{T}, \\
k_{r}^{*} & =-0.380228
\end{aligned}
$$

The above $K^{*}$ and $k_{r}^{*}$ are the values that would make up the nominal controller,

$$
u(t)=K^{* T} x(t)+k_{r}^{*} r(t)
$$

which would control the output $\psi$ in a worst case scenario icing encounter. However, this nominal controller is unknown because the system and input matrices for a worst case scenario icing encounter are unknown. Therefore, the estimates $K(t)$ and 
$k_{r}(t)$ of $K^{*}$ and $k_{r}^{*}$ will be determined from adaptive laws and used in the adaptive controller structure

$$
u(t)=K^{T}(t) x(t)+k_{r}(t) u(t) .
$$

For the purposes of the simulation, the ideal gains for the lateral-directional state space model in no icing conditions were calculated. They are used to show a fixed gain controller cannot handle the changes in aerodynamics that result from ice accretion on an aircraft. The gains are calculated using (4.7) and are presented as

$$
\begin{aligned}
K^{*} & =\left[\begin{array}{lllll}
-0.0711199 & -0.0000506468 & 0.822802 & 0.182612 & 0.380263
\end{array}\right]^{T}, \\
k_{r}^{*} & =-0.380228
\end{aligned}
$$

The simulation for the fixed gain controller for the output tracking of $\psi$ is shown in figure 5.9. The adaptive simulations for $\psi$ are presented in figures 5.10, 5.11, 5.12, and 5.13 . 


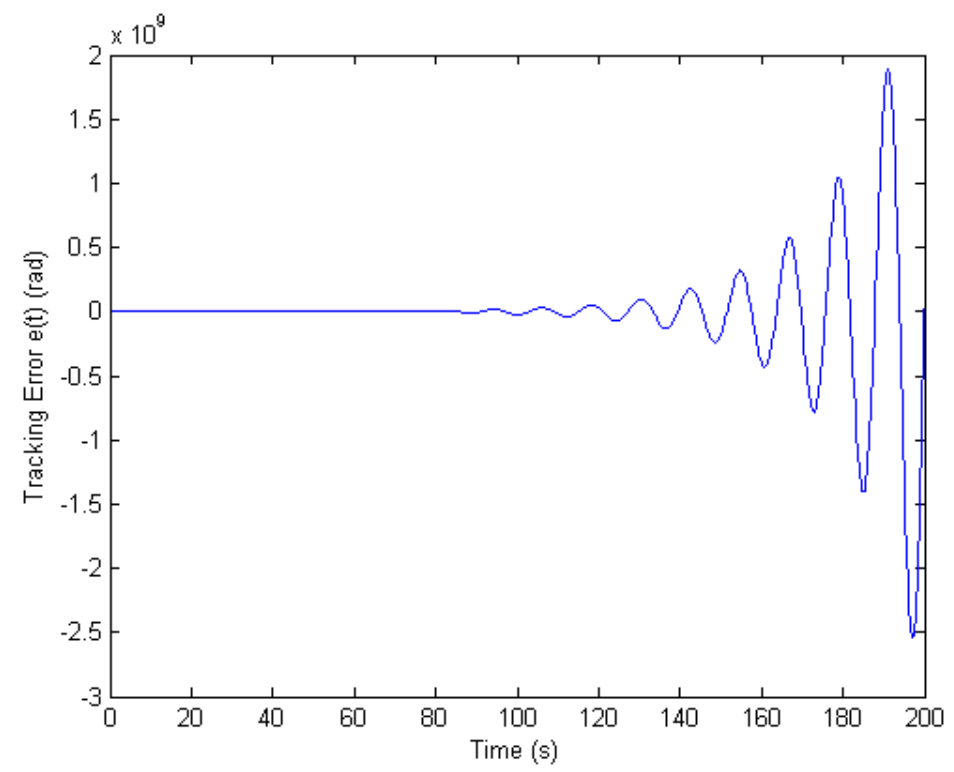

Figure 5.9: Output tracking error for the fixed gain control of the heading angle, $\psi$, in a worst case scenario icing encounter at $t=30 \mathrm{~s}$.

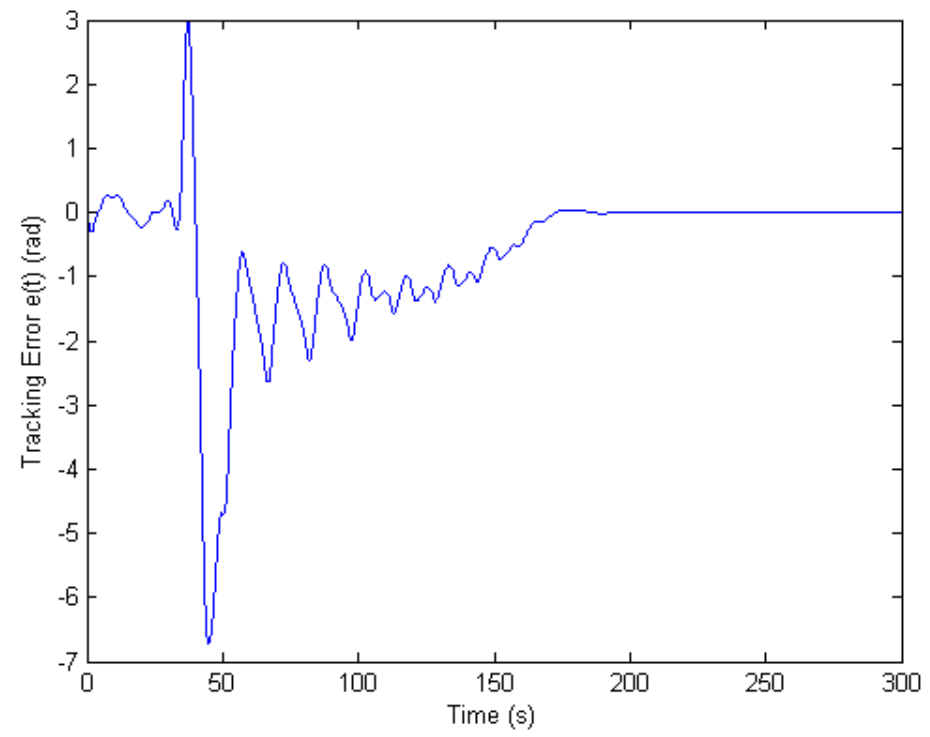

Figure 5.10: Output tracking error for the adaptive control of the heading angle, $\psi$, in a worst case scenario icing encounter at $t=30 \mathrm{~s}$. 

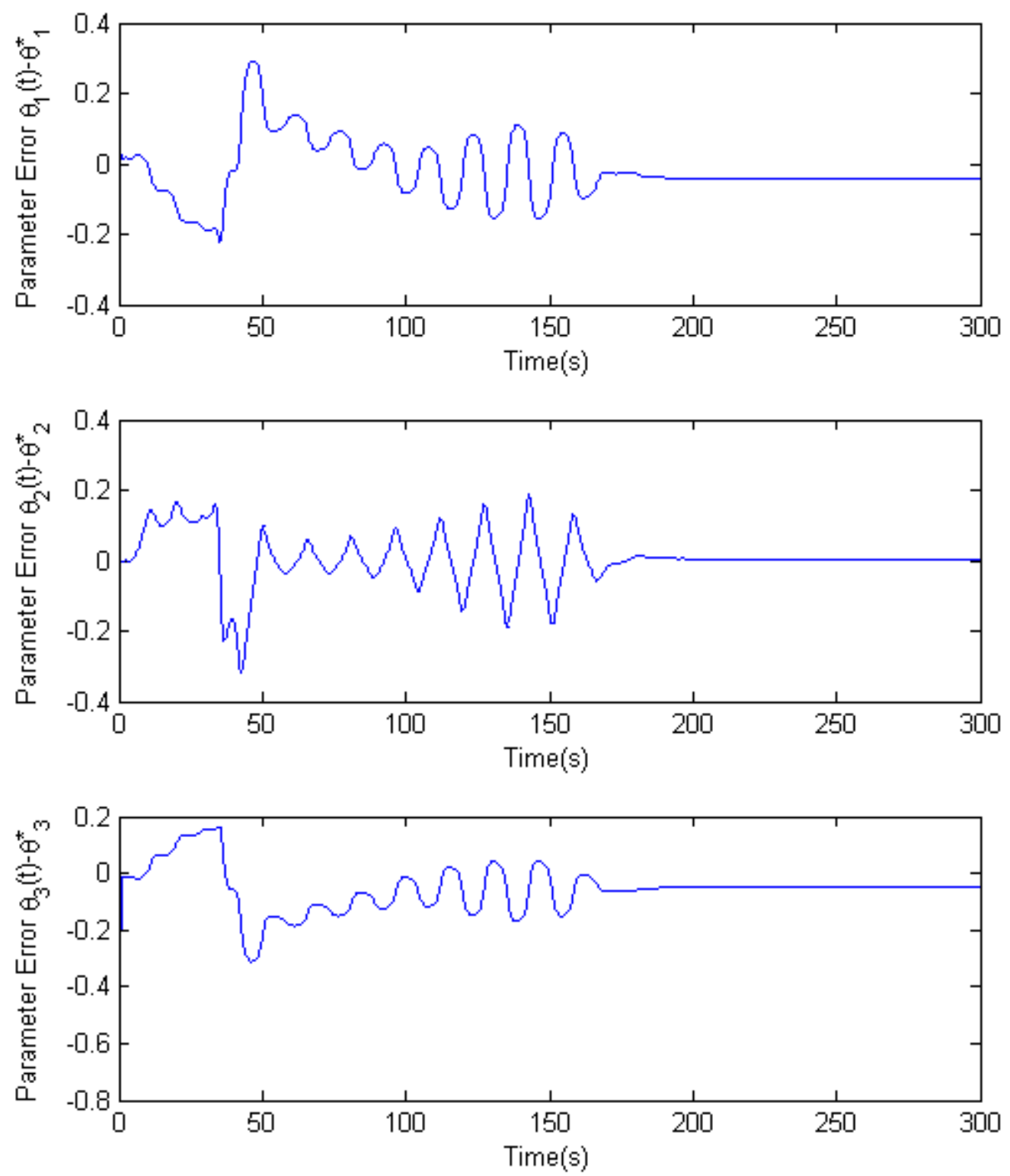

Figure 5.11: Parameter errors for $\theta_{1}, \theta_{2}$, and $\theta_{3}$ for the adaptive control of the heading angle, $\psi$, in a worst case scenario icing encounter at $t=30 \mathrm{~s}$. 

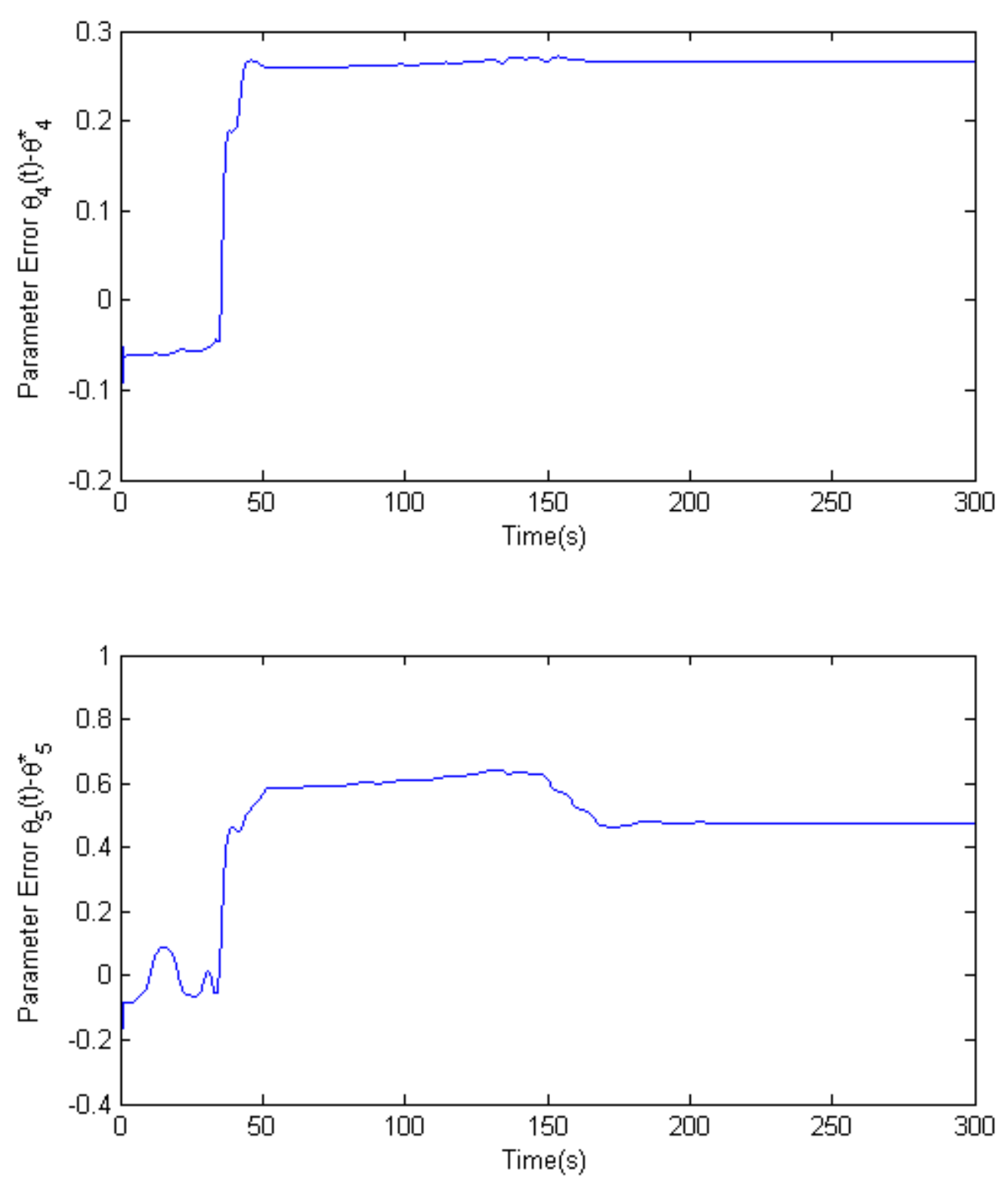

Figure 5.12: Parameter errors for $\theta_{4}$ and $\theta_{5}$ for the adaptive control of the heading angle, $\psi$, in a worst case scenario icing encounter at $t=30 \mathrm{~s}$. 

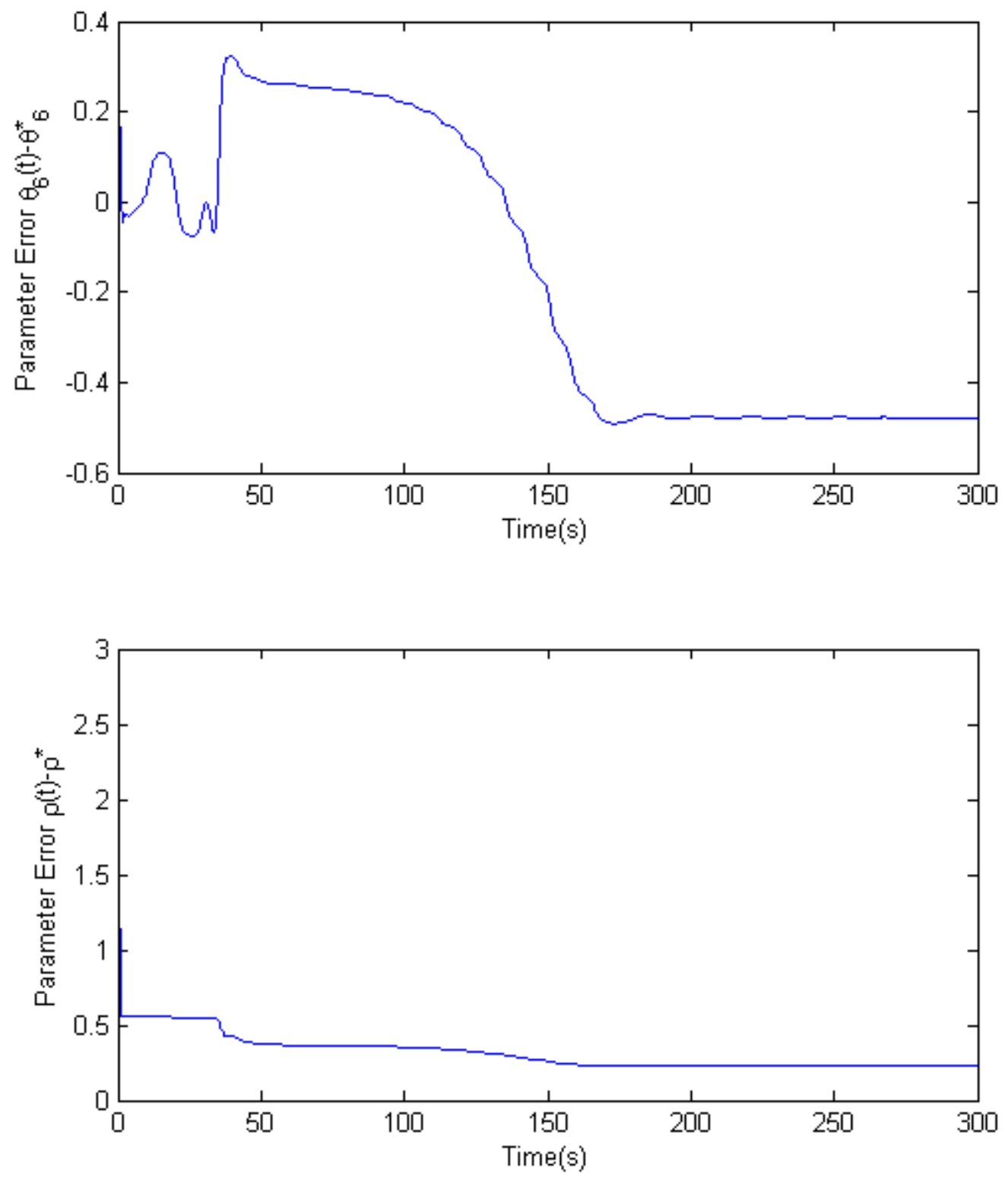

Figure 5.13: Parameter errors for $\theta_{6}$ and $\rho$ for the adaptive control of the heading angle, $\psi$, in a worst case scenario icing encounter at $t=30 \mathrm{~s}$. 


\section{Discussion}

Figure 5.9 clearly shows that there is no possiblity of the ideal fixed gain controller to regain control of the aircraft. Also, it is obvious that no matter what action is taken by the autopilot, or flight crew, the aircraft will become uncontrollable. Due to the scaling of the plot, it is hard to tell exactly the point at which the aircarft would lose control entirely. However, considering the scaling, it is reasonable to assume that the aircraft has become uncontrollable anytime after 30 seconds.

The adaptive controller handles the change in flight dynamics well bringing the tracking error to zero. Again, the time it takes for the tracking error to come to zero can be altered by a change of the adaptation gains $\Gamma$ and $\gamma$.

Notice that the parameter errors do not go to zero in this case either. However, all parameters converge to a constant value relatively close to the ideal parameters. For more information on the factors governing parameter convergence see [20]. 


\subsection{Worst Case Scenario Horizontal Tail Icing}

\subsubsection{Longitudinal Dynamic Model}

\section{Situation}

For this simulation the situation is the same as that in section 5.1.1. However, now there is an initial instantaneous freezing of liquid water droplets followed by a constant increase in ice accretion on only the vulnerable surfaces of the horizontal tail. The terms of the longitudinal dynamics that are effected are discussed in section 3.3.3.

\section{Simulation Results}

The outputs $\alpha$ and $\theta$, in a worst case scenario horizontal tail icing condition, are of interest for adaptive control. These outputs are dependent upon the input originating from $\delta_{e}$. The ideal gains for the respective controllers were calculated using the matching condition (4.7). The values for these ideal gains were presented in section 4.2.1 and are reproduced here as

$$
\begin{aligned}
K^{*} & =\left[\begin{array}{llll}
-0.0120029 & -1.39002 & 8.93851 & -0.0286146
\end{array}\right]^{T}, \\
k_{r}^{*} & =-9.23361
\end{aligned}
$$

for $\alpha$ and

$$
\begin{aligned}
K^{*} & =\left[\begin{array}{llll}
-0.0000578944 & -0.992183 & -0.090303 & 0.115851
\end{array}\right]^{T}, \\
k_{r}^{*} & =-0.115821
\end{aligned}
$$


for $\theta$. These two sets of $K^{*}$ and $k_{r}^{*}$ are the values that would make up the nominal controller,

$$
u(t)=K^{* T} x(t)+k_{r}^{*} r(t),
$$

that would control the outputs $\alpha$ and $\theta$ in a worst case scenario horizontal tail icing encounter, respectively. However, this nominal controller is unknown because the system and input matrices for a worst case scenario horizontal tail icing encounter are unknown. Therefore, the estimates $K(t)$ and $k_{r}(t)$ of $K^{*}$ and $k_{r}^{*}$ will be determined from adaptive laws and used in the adaptive controller structure

$$
u(t)=K^{T}(t) x(t)+k_{r}(t) u(t) .
$$

For the purposes of the simulation, the ideal gains for the longitudinal state space model in no icing conditions were calculated. They are used to show how a fixed gain controller cannot handle the changes in aerodynamics that result from ice accretion on an aircraft. The gains are calculated using (4.7) and are the same as (5.9), (5.10), (5.11), and (5.12) in section 5.1.1.

The simulations for the fixed gain controllers for the output tracking of $\alpha$ and $\theta$ are shown in figures 5.14 and 5.18, respectively. The adaptive simulations for $\alpha$ are presented in figures 5.15, 5.16, and 5.17. Likewise, the adaptive simulations for $\theta$ are presented in figures 5.19, 5.20, and 5.21. 


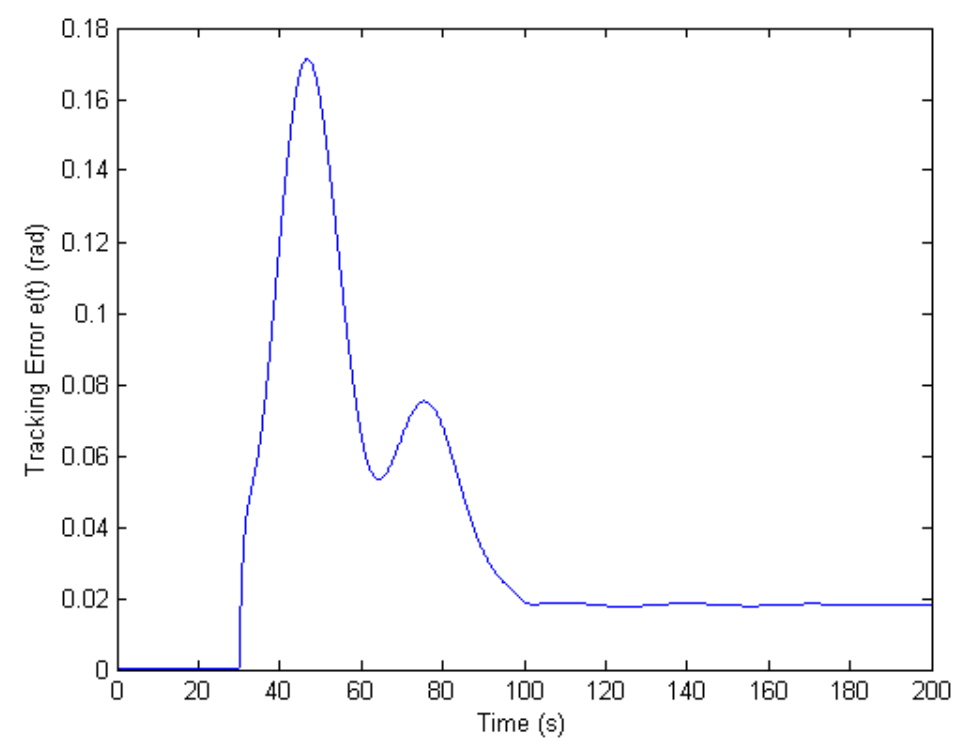

Figure 5.14: Output tracking error for the fixed gain control of the angle of attack, $\alpha$, in a horizontal tail worst case scenario icing encounter at $t=30 \mathrm{~s}$.

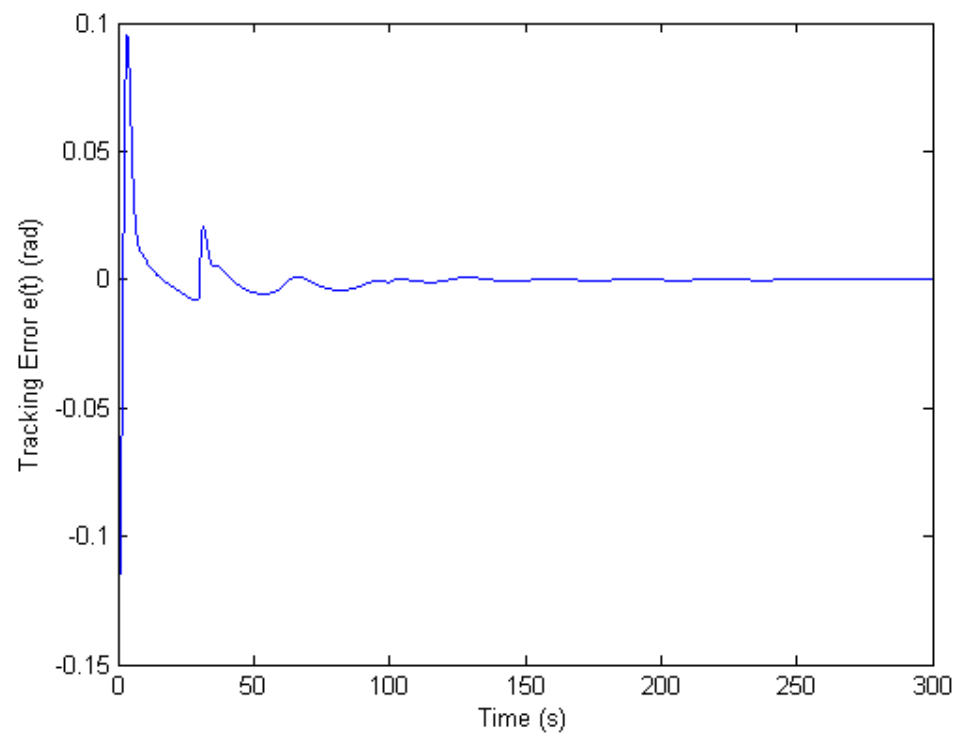

Figure 5.15: Output tracking error for the adaptive control of the angle of attack, $\alpha$, in a horizontal tail worst case scenario icing encounter at $t=30 \mathrm{~s}$. 

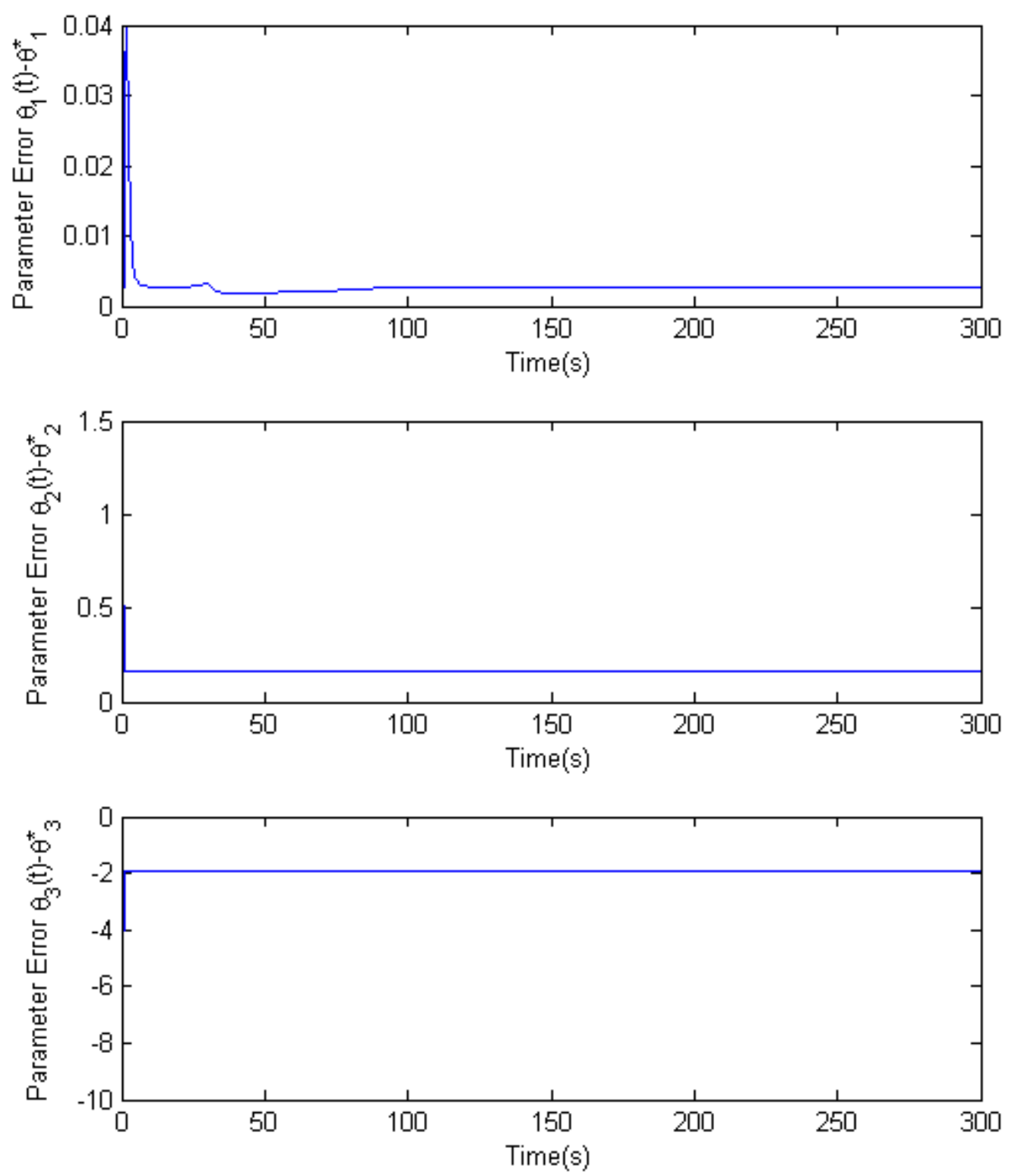

Figure 5.16: Parameter errors for $\theta_{1}, \theta_{2}$, and $\theta_{3}$ for the adaptive control of the angle of attack, $\alpha$, in a horizontal tail worst case scenario icing encounter at $t=30 \mathrm{~s}$. 

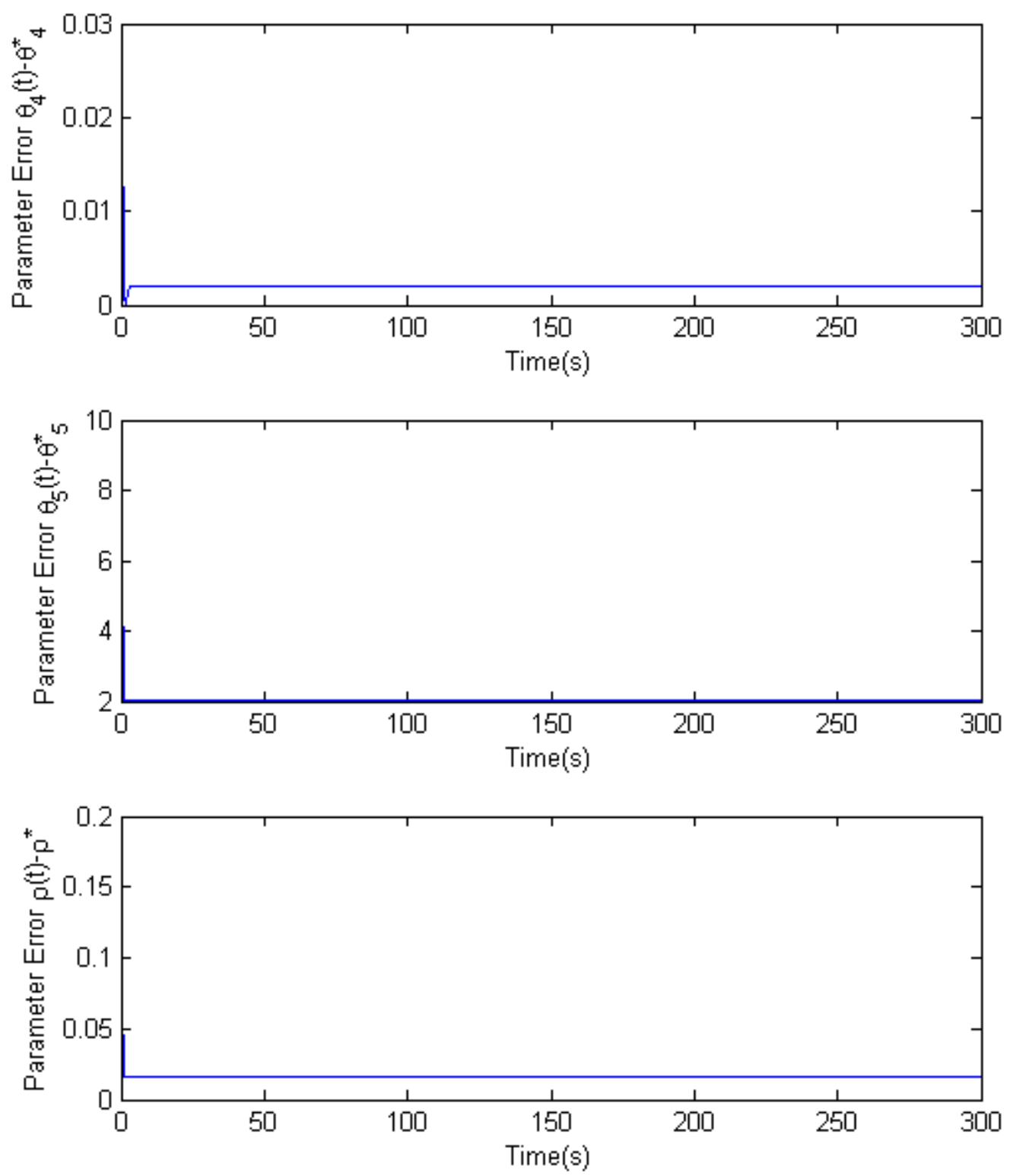

Figure 5.17: Parameter errors for $\theta_{4}, \theta_{5}$, and $\rho$ for the adaptive control of the angle of attack, $\alpha$, in a horizontal tail worst case scenario icing encounter at $t=30 \mathrm{~s}$. 


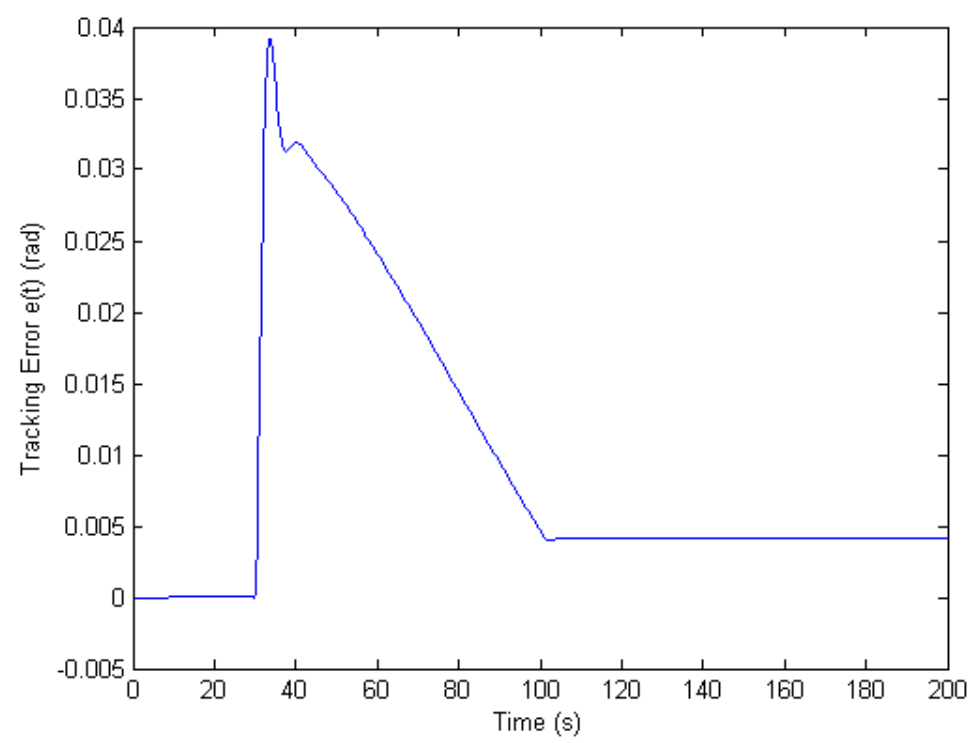

Figure 5.18: Output tracking error for the fixed gain control of the pitch attitude angle, $\theta$, in a horizontal tail worst case scenario icing encounter at $t=30 \mathrm{~s}$.

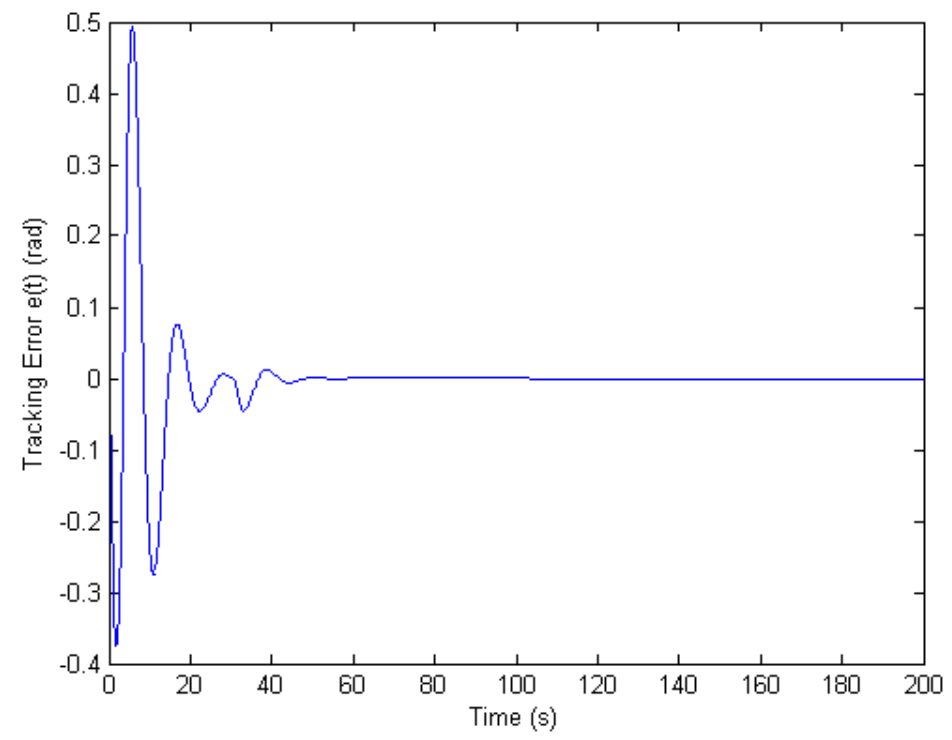

Figure 5.19: Output tracking error for the adaptive control of the pitch attitude angle, $\theta$, in a horizontal tail worst case scenario icing encounter at $t=30 \mathrm{~s}$. 

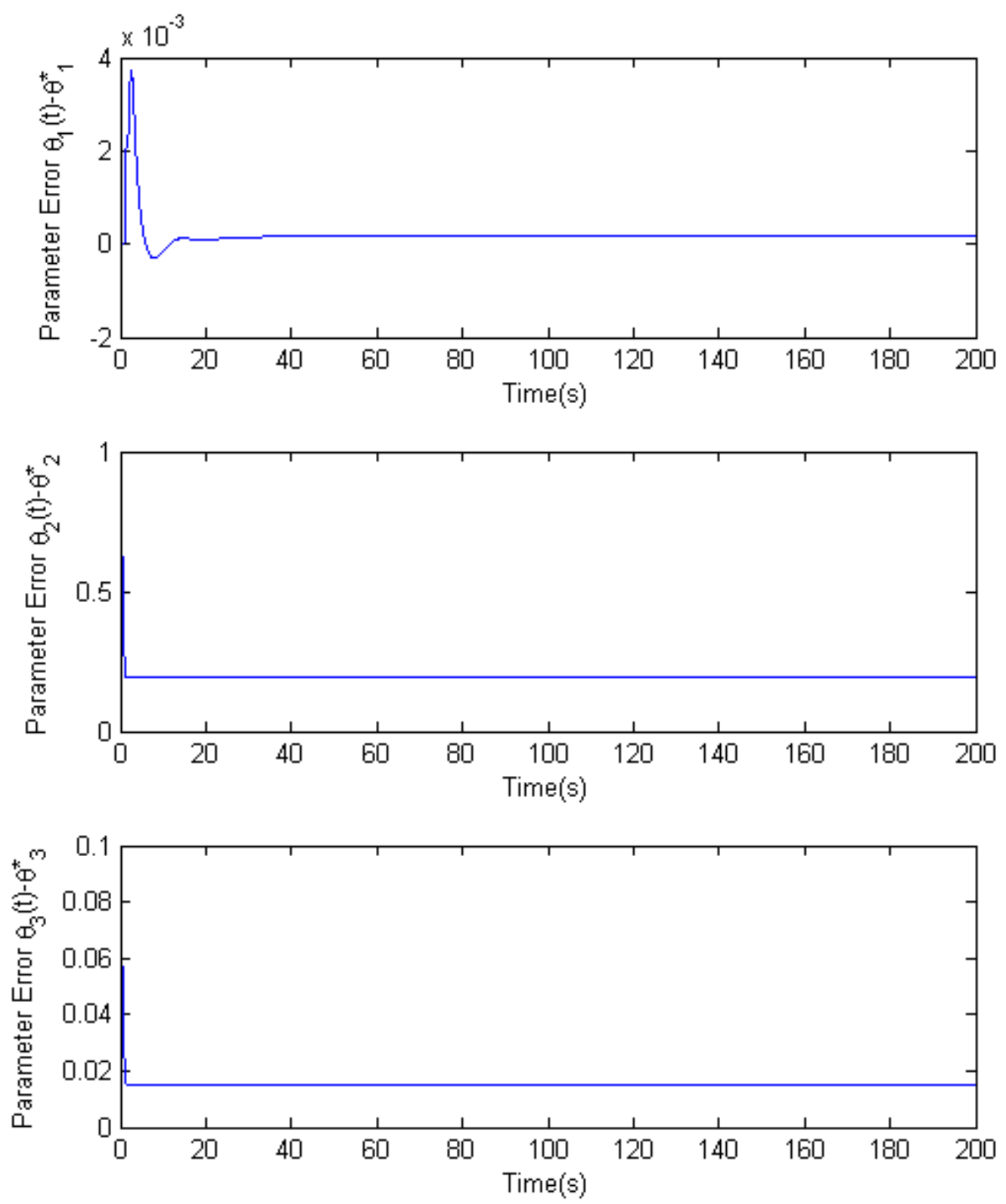

Figure 5.20: Parameter errors for $\theta_{1}, \theta_{2}$, and $\theta_{3}$ for the adaptive control of the pitch attitude angle, $\theta$, in a horizontal tail worst case scenario icing encounter at $t=30 \mathrm{~s}$. 

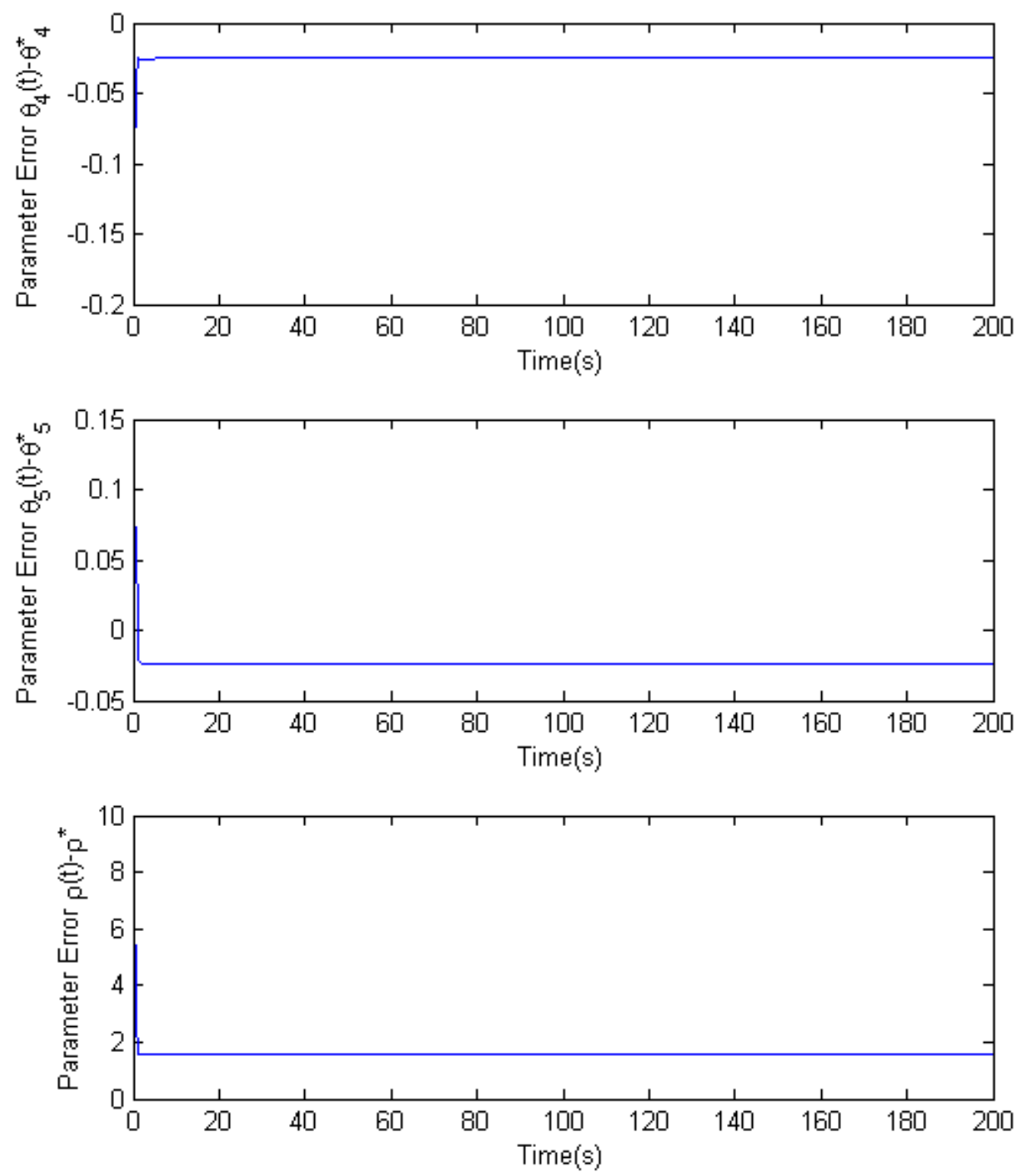

Figure 5.21: Parameter errors for $\theta_{4}, \theta_{5}$, and $\rho$ for the adaptive control of the pitch attitude angle, $\theta$, in a horizontal tail worst case scenario icing encounter at $t=30 \mathrm{~s}$. 


\section{Discussion}

In figures 5.14 and 5.18 the behavior of the fixed gain controllers are similar to that of the fixed gain controllers in figures 5.1 and 5.5. It is apparent that the fixed gain controllers are able to maintain reasonable control of the aircaft. The tracking error for $\alpha$ and $\theta$ appears to settle at a constant value towards the end of the simulation. The initial increase coupled with the time it takes to decrease the tracking error would pose a great challenge to the autopilot. Due to the rate at which the tracking error increases it is likely the autopilot would disconnect.

The tracking error simulation of fig. 5.14 does not adequately reflect the typical behavior of heavy tail icing on an aircraft. Typically, the aircraft would tend to pitch downward unexpectedly with the possibility for a regain in control unlikely. This would be especially true in a worst case scenario horizontal tail icing encounter. It is possible that the Cessna 208 examined here could experience little effects due to horizontal tail icing. That being said, there have been many reports and suspicions related to Cessna 208 accidents that point to the possible inaccuracy of the icing effects model used in this research [39].

The adaptive controller handles the change in flight dynamics well bringing the tracking error to zero for both the outputs $\alpha$ and $\theta$. Again, with an appropriate choice of $\Gamma$ and $\gamma$ the tracking error could converge to zero much faster. It is of importance to note as well that all parameters converge to a constant value relatively close to the ideal parameters. Again, parameter convergence is not guaranteed, nor needed, for reasons discussed in [20]. 


\subsubsection{Lateral-Directional Dynamic Model}

\section{Situation}

For this simulation the situation is the same as that in section 5.1.1. However, now there is an initial instantaneous freezing of liquid water droplets followed by a constant increase in ice accretion on the vulnerable surfaces of the horizontal tail. The terms of the lateral-directional dynamics that are effected are discussed in section 3.3.3.

\section{Simulation Results}

The output $\psi$, in a worst case scenario horizontal tail icing condition, is of interest for adaptive control. This output is dependent upon the input originating from $\delta_{R}$. The ideal gains for this controller were calculated using the matching condition (4.7). The values for these ideal gains are presented in section 4.2.1 and are reproduced here as

$$
\begin{aligned}
K^{*} & =\left[\begin{array}{lllll}
-0.539621 & 0.0284752 & 0.04554 & 0.146255 & 0.404578
\end{array}\right]^{T}, \\
k_{r}^{*} & =-0.380228
\end{aligned}
$$

The above $K^{*}$ and $k_{r}^{*}$ are the values that would make up the nominal controller,

$$
u(t)=K^{* T} x(t)+k_{r}^{*} r(t)
$$

which would control the output $\psi$ in a worst case scenario icing encounter. However, this nominal controller is unknown because the system and input matrices for a 
worst case scenario icing encounter are unknown. Therefore, the estimates $K(t)$ and $k_{r}(t)$ of $K^{*}$ and $k_{r}^{*}$ will be determined from adaptive laws and used in the adaptive controller structure

$$
u(t)=K^{T}(t) x(t)+k_{r}(t) u(t) .
$$

For the purposes of the simulation, the ideal gains for the lateral-directional state space model in no icing conditions were calculated. They are used to show how a fixed gain controller cannot handle the changes in aerodynamics that result from ice accretion on an aircraft. The gains are calculated using (4.7) and are the same as (5.17) and (5.18) in section 5.1.2.

The simulation for the fixed gain controller for the output tracking of $\psi$ is shown in figure 5.22. The adaptive simulations for $\psi$ are presented in figures 5.23, 5.24, 5.25 , and 5.26 . 


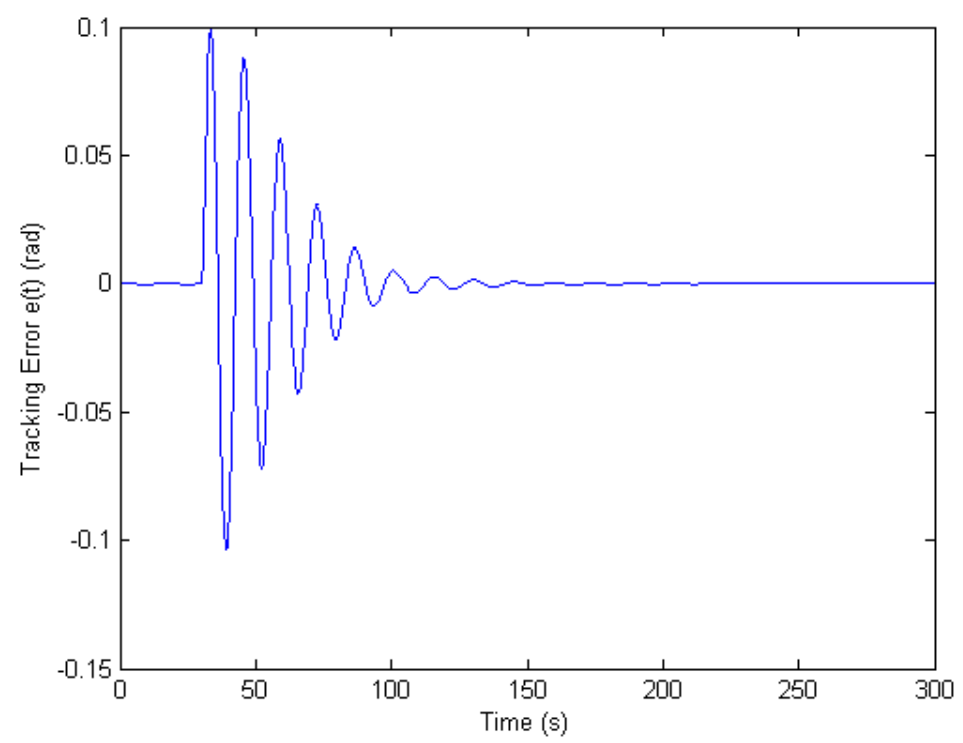

Figure 5.22: Output tracking error for the fixed gain control of the heading angle, $\psi$, in a horizontal tail worst case scenario icing encounter at $t=30 \mathrm{~s}$.

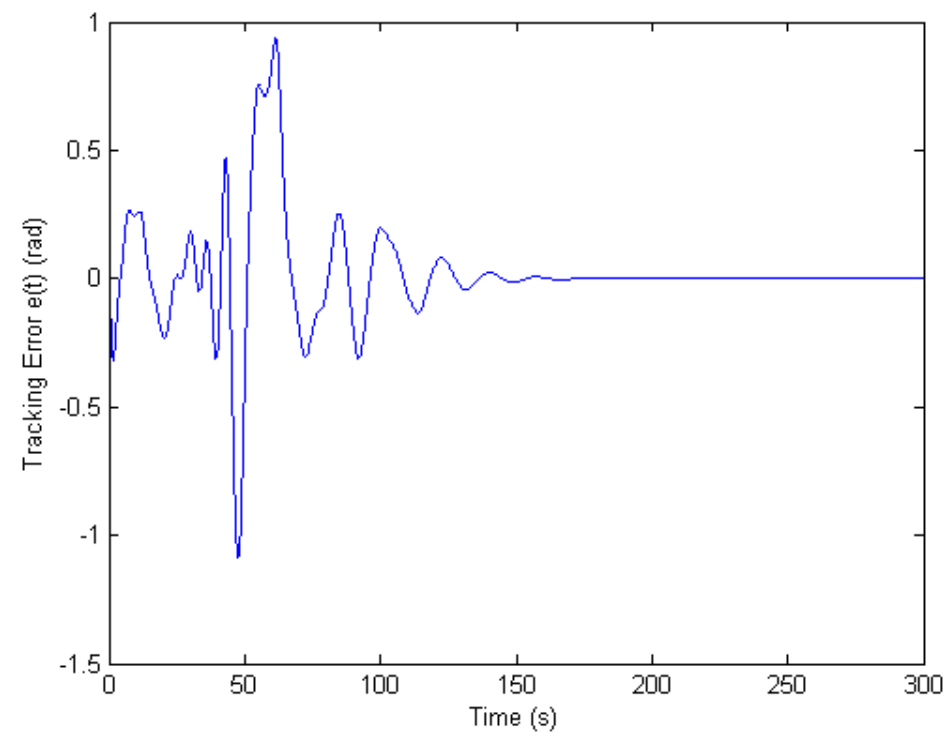

Figure 5.23: Output tracking error for the adaptive control of the heading angle, $\psi$, in a horizontal tail worst case scenario icing encounter at $t=30 \mathrm{~s}$. 

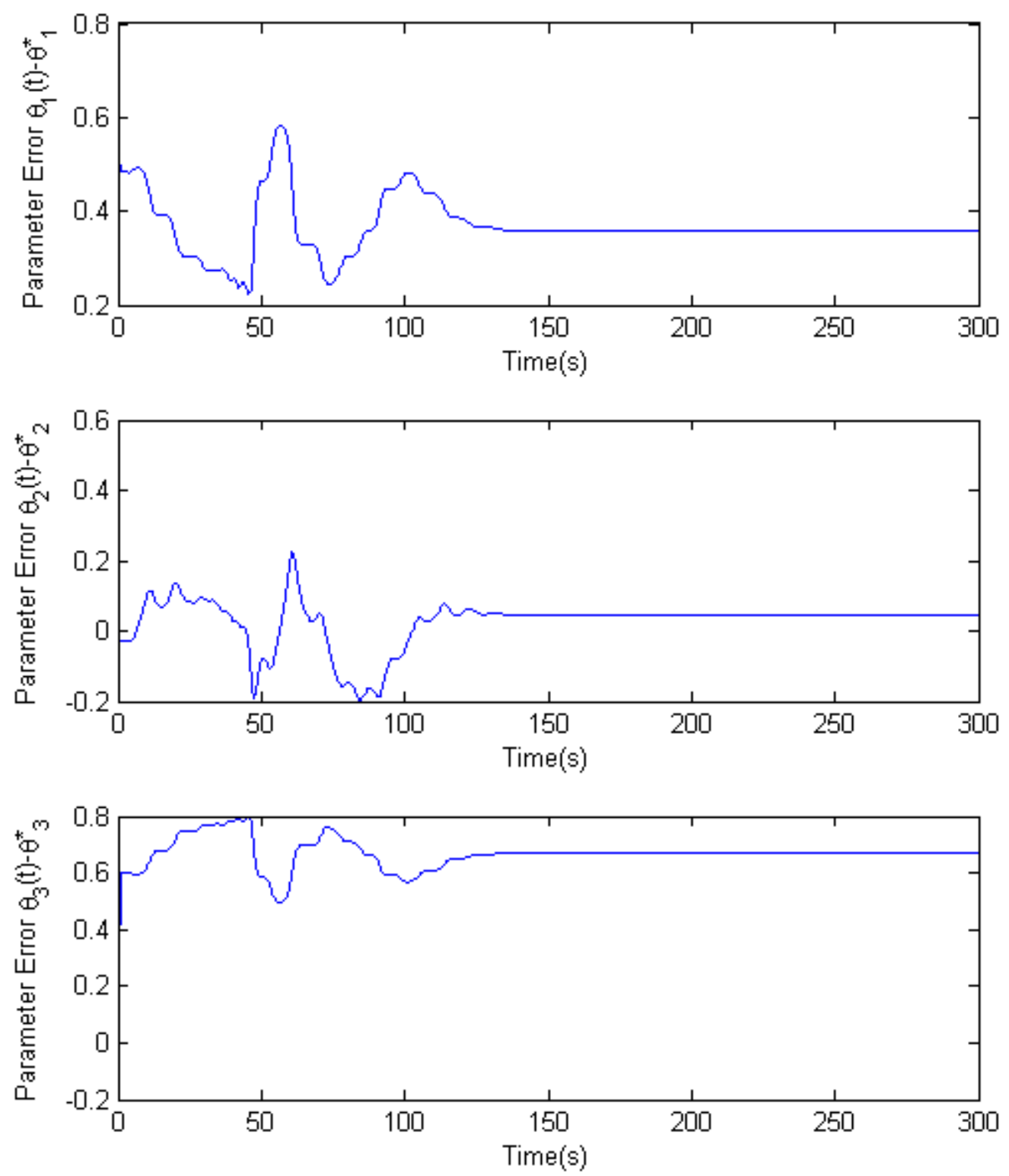

Figure 5.24: Parameter errors for $\theta_{1}, \theta_{2}$, and $\theta_{3}$ for the adaptive control of the heading angle, $\psi$, in a horizontal tail worst case scenario icing encounter at $t=30 \mathrm{~s}$. 

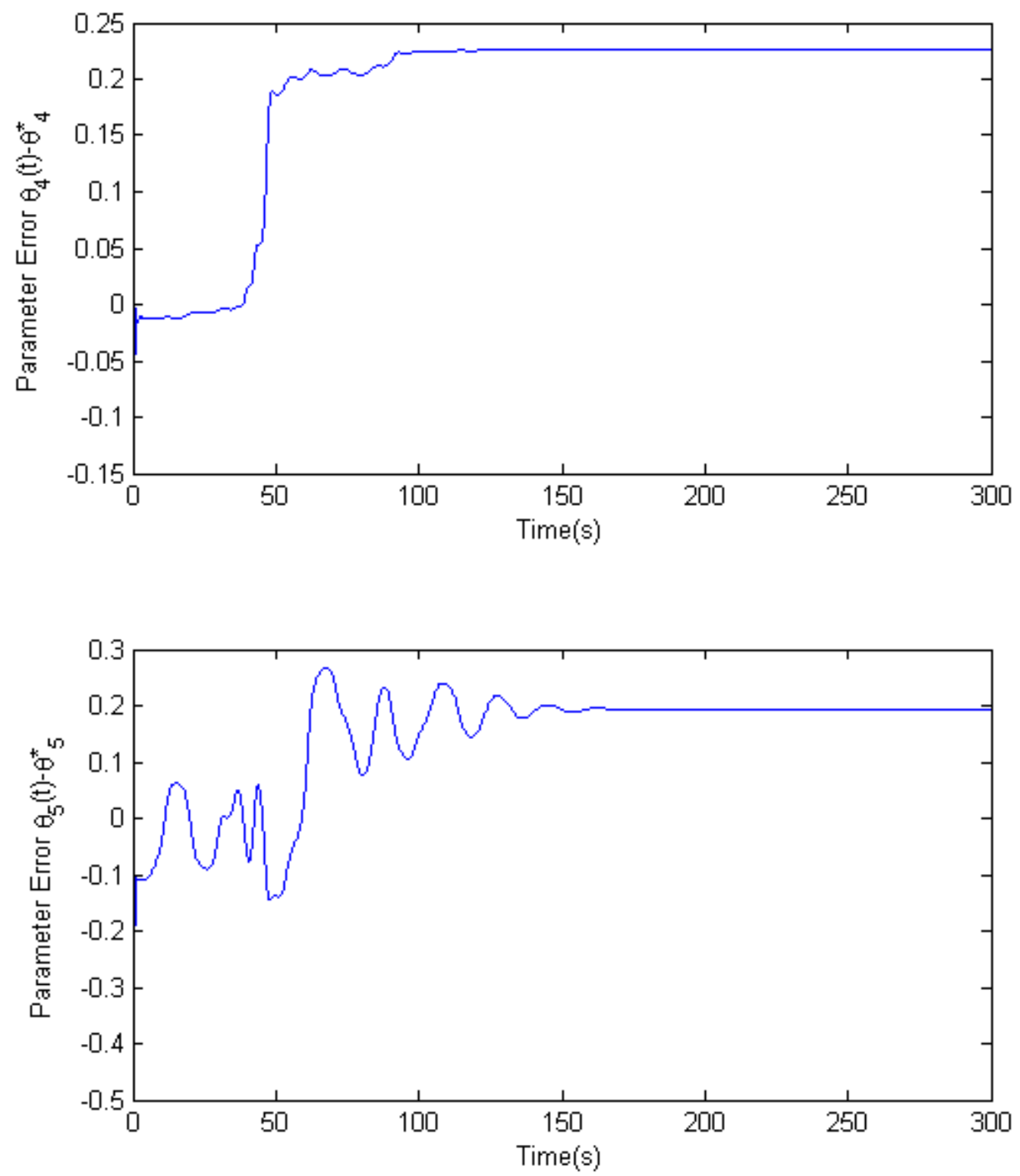

Figure 5.25: Parameter errors for $\theta_{4}$ and $\theta_{5}$ for the adaptive control of the heading angle, $\psi$, in a horizontal tail worst case scenario icing encounter at $t=30 \mathrm{~s}$. 

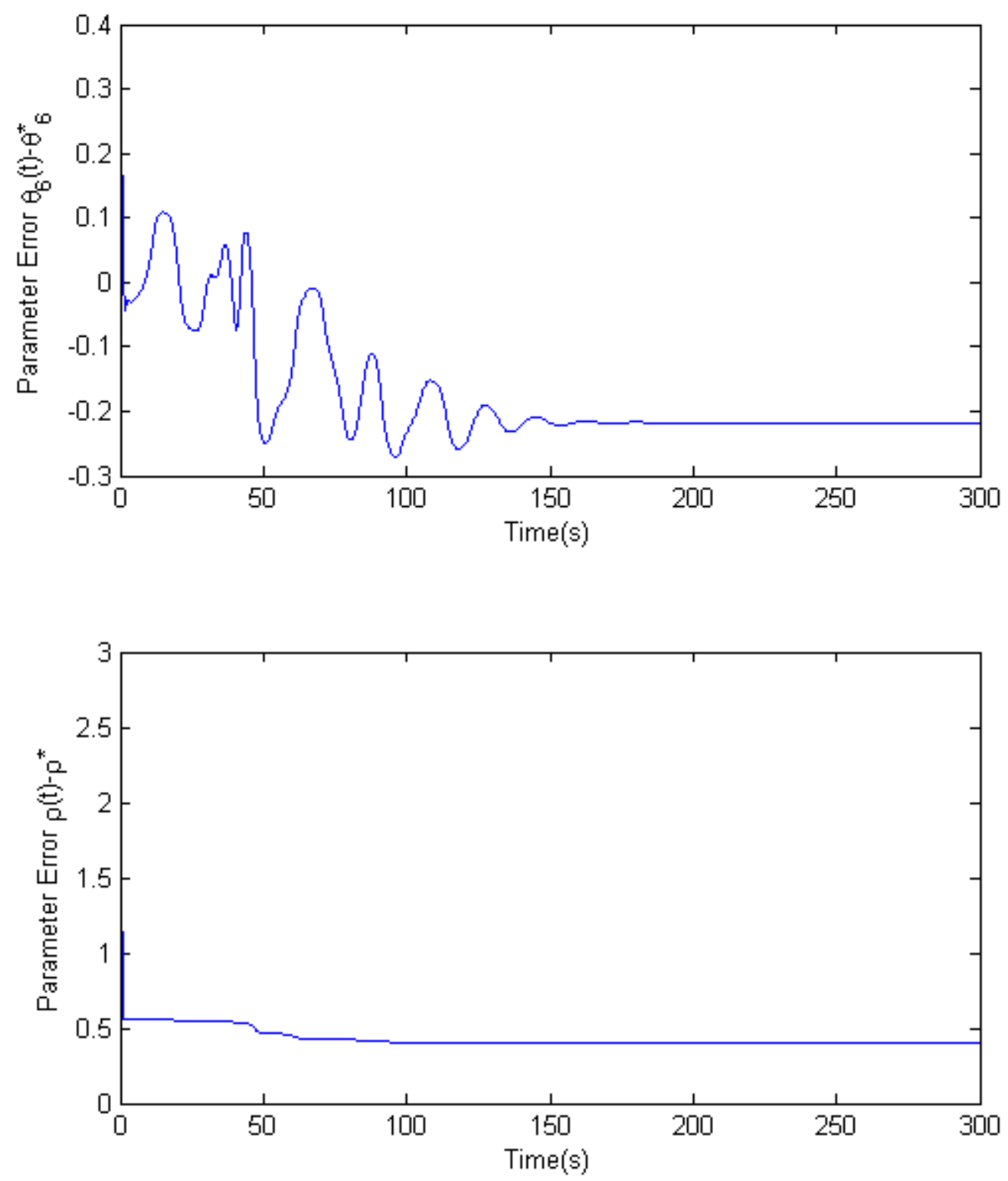

Figure 5.26: Parameter errors for $\theta_{6}$ and $\rho$ for the adaptive control of the heading angle, $\psi$, in a horizontal tail worst case scenario icing encounter at $t=30 \mathrm{~s}$. 


\section{Discussion}

In fig. 5.22 the fixed gain controller is able to bring the tracking error to zero. This would seem to be a surprising result considering the behavior of the fixed gain controller in fig. 5.9. However, it is important to remember that only the horizontal stabilizer of the tail has been modeled to accumulate ice. Therefore, the result of the fixed gain controller is not as astonishing as it seems to be.

It is likely the flight crew would notice the small oscillations in heading angle

during flight. However, due to the atomity of them it would unlikely be the cause of any concern. With that being said, due to the rapid change in heading angle there is still a possibility that the autopilot may disconnect, even though the oscillations aren't large. As stated before, if a pilot were to be overaggressive with commanded inputs the fixed gain controller may not be able to cope.

The adaptive controller handles the change in flight dynamics well bringing the tracking error to zero. Again, the time it takes for the tracking error to come to zero can be altered by a change of the adaptation gains $\Gamma$ and $\gamma$. Notice that the parameter errors do not go to zero in this case either. However, all parameters converge to a constant value relatively close to the ideal parameters. For more information on the factors governing parameter converge see [20]. 


\subsection{Summary}

In this chapter simulations of the longitudinal and lateral-directional dynamics in a worst case scenario and a worst case scenario horizontal tail icing encounter were presented. It was found that the adaptive controller was able to reduce the tracking error to zero for all cases. It was mentioned, but not shown, that with an adjustment of the adaptation gains the speed at which the tracking error converges can be controlled. A physical interpretation of the simulations was given following the plots of each scenario. 


\section{Chapter 6}

\section{Conclusions and Future Work}

\subsection{Summary and Conclusions}

In this thesis, the motivations for committing to this research were proposed and discussed. Following this, an approach to modeling aircraft icing was presented. Methods for modeling a totally iced aircraft and horizontal tail icing were explained. The basics of adaptive control theory were presented along with a more detailed account of the design for output tracking. It was found that output tracking could be achieved for some outputs of both the longitudinal and lateral-directional models in a worst case scenario and worst case scenario horizontal tail icing encounter.

A disappointing result in this thesis was the inability to satisfy the design conditions so that the roll angle, $\phi$, could be controlled. The uncommanded roll due to aileron hinge moment reversal is a major cause of concern since many aviation accidents due to icing are caused by it. A different approach to designing an adaptive 
control system might yield controllability of $\phi$. Granted, it is possible that with the control of the angle of attack, $\alpha$, the pitch attitude angle, $\theta$, and the heading angle, $\psi$, uncommanded rolls of the aircraft may pose less of a problem. However, this would need to be verified in a flight simulator.

\subsection{Future Research Topics}

Future research will largely depend on the accuracy of the aircraft models. Systematic ways of predicting how ice accretion will form and the resultant aerodynamic effects is the subject of much ongoing research. With an improved model, more powerful adaptive control strategies may be employed.

It has been assumed throughout this thesis that knowledge of the aircraft states are available. This may not always be the case. Without knowledge of the states an adaptive control design cannot be achieved. To counter this problem a state observer would need to be employed to determine the states. Another option would be to attempt a design utilizing output feedback instead of state feedback.

The response time of the adaptive controller could pose a potential research area. There may exist a way to update the gains $\Gamma$ and $\gamma$ online with the adaptive update laws to further minimize the time it takes for the tracking error to approach zero. It is possible that an approach similar to the normalized least-squared algorithm to update $\Gamma$ and $\gamma$ would be possible. This would be crucial to maintaining desired flight characteristics.

Another topic for further research would be to analyze the adaptive control of a 
situation where ice accretion has developed on only one wing. The cause of such a scenario would be the failure of only one of the wing's de-icing mechanisms on an aircraft. This has been modeled as a step disturbance in [4], [3], and [5].

This research was based upon models describing the Cessna 208 Super Cargomaster, which is a small aircraft that is incapable of flying at high speeds and altitudes. This brings up the question as to whether larger planes that are capable of high speeds and altitudes are as susceptible to ice accretion as small planes. In a discussion with Major Larry Crews (USAF Ret.) about the potential for research on larger planes, it was conferred that the issue of icing was less of a problem. Major Crews flew a variety of small and large aircraft during his military career that included the Boeing B-52 Stratofortress, Boeing KC-135 Stratotanker, Cessna T-37 Tweet, and Northrop T-38 Talon. At the time, none of these planes were outfitted with any kind of de-icing equipment. In his post military career he flew the small Fairchild SA-227 Metroliner and the large Boeing 747.

The unlikelyhood of ice accretion on the larger planes was mainly due to the higher altitudes they are capable of maintaining as well as the speed at which they can travel. When landing, and icing conditions are no longer avoidable, the standard procedure is to land as quickly as possible. The amount of ice accretion that it takes for a large aircraft to be effected by icing is very difficult to accumulate in the time it takes to descend in altitude and land. That being said, Major Crews noted that though aerodynamic effects of icing in faster moving planes would be unlikely, engine icing is a great threat. He recalled that the small T-38 was infamous for such behavior. The problem of engine icing would be a potential area for research for 
both small and large fast moving planes.

To truly be able to analyze the applicability of an adaptive controller to handle an icing encounter, flight data would need to be collected and used to simulate an adaptive control scheme. With this data an adaptive controller could be designed and its performance evaluated. Such information exists, though it is based on wind tunnel tests such as those in [54], and there is ongoing research into developing flight simulators that rely solely on it for pilot training purposes. Outfitting such a simulator with an adaptive controller would be a way of analyzing the controller's usefullness as well as answering any performance questions in regard to the controller. 


\section{References}

[1] Anon., "Cessna Aircraft Company Model 208B Information Manual," Cessna Aircraft Co., Wichita, KS, June 2008.

[2] Anon., "Super Cargomaster Caravan Specification and Description Effective Serial Number 208B-5000 and on," Cessna Aircraft Co., Wichita, KS, January 2013.

[3] Amanda Lampton and John Valasek, "Prediction of icing effects on the dynamic response of light airplanes," Journal of Guidance, Control, and Dynamics, vol. 30, no. 3, pp.722-732, 2007.

[4] Amanda Lampton and John Valasek, "Prediction of icing effects on the lateral/directional stability and control of light airplanes," AIAA Atmospheric Flight Mechanics Conference and Exhibit, Keystone, CO, Aug. 2006.

[5] Amanda Lampton and John Valasek, "Prediction of icing effects on the coupled dynamic response of light airplanes," Journal of Guidance, Control, and Dynamics, vol. 31, no. 3, pp.656-673, 2008. 
[6] A. Tremblay, S. G. Cober, A. Glazer, and G. Isaac, "An intercomparison of mesoscale forecasts of aircraft icing using SSM/I retrievals," Journal of Weather and Forecasting, vol. 11, pp. 66-77, 1995.

[7] B. Landsberg et al., 2008 April, Safety Advisor - Weather No. 1 [Online], Air Safety Foundation (AOPA). Available: http://flighttraining.aopa.org/pdfs/SA11 Aircraft_Icing.pdf

[8] B. N. Pamadi, Performance, Stability, Dynamics, and Control of Airplanes, American Institute of Aeronautics and Astronautics Inc., Reston, VA, 2004.

[9] Benjamin C. Kuo, Digital Control Systems, $2^{\text {nd }}$ Edition, Oxford University Press Inc., New York, NY, 1992.

[10] Chi-Tsong Chen, Linear System Theory and Design, $3^{\text {rd }}$ Edition, Oxford University Press Inc., New York, NY, 1999.

[11] D. Cook "Relationships of ice shapes and drag to icing condition dimensionless parameters," Proceedings of the $38^{\text {th }}$ AIAA Aerospace Sciences Meeting and Exhibit, Reno, NV, Jan. 2000.

[12] D. Pokhariyal, M.B. Bragg, T. Hutchison, and J. Merret, "Aircraft flight dynamics with simulated ice accretion," Proceedings of the $39^{\text {th }}$ AIAA Aerospace Sciences Meeting and Exhibit, Reno, NV, Jan. 2001.

[13] Daniel Shields, A Modeling Study of Ice Accretion on a NACA 4412 Airfoil. Thesis, Rensselaer Polytechnic Institute. Hartford, Connecticut 2011. 
[14] E. Weener. 2011, February 1. Lessons from Icing Accidents and Incidents [Online]. Available: https://www.ntsb.gov/doclib/speeches/weener/weener_020111. pdf

[15] Ethirajan Rathakrishnan, Theoretical Aerodynamics John Wiley and Sons Singapore Pte. Ltd., Solaris South Tower, Singapore, 2013.

[16] Eugene Lavretsky and Kevin A. Wise, Robust and Adaptive Control with Aerospace Applications, Springer, London, United Kingdom, 2013.

[17] Federal Aviation Administration. (July 17, 1996). Effect of icing on aircraft control and airplane deice and anti-ice systems (Advisory Circular 91-51A). William J. White.

[18] G. F. Franklin, J. D. Powell, and A. Emami-Naeini, Feedback Control of Dynamic Systems, $6^{\text {th }}$ Edition, Pearson Education Inc., Upper Saddle River, NJ, 2009.

[19] G. Kowaleczko and M. Wachaczenko, "Aircraft dynamics during flight in icing conditions," Journal of Theoretical and Applied Mechanics, Vol. 50, No. 1 , pp. $269-284$.

[20] G. Tao, Adaptive Control Design and Analysis, John Wiley and Sons Inc., Hoboken, NJ, 2003.

[21] Gareth Williams, Linear Algebra with Applications, $4^{\text {th }}$ Edition, Jones and Bartlett Publishers Inc., Sudbury, MA, 2001. 
[22] H. M. Gurbacki and M. B. Bragg, "Unsteady aerodynamic measurements on an iced airfoil," Proceedings of the $40^{\text {th }}$ AIAA Aerospace Sciences Meeting and Exhibit, Reno, NV, Jan. 2002.

[23] Hassan K. Khalil, Nonlinear Systems, $3^{\text {rd }}$ Edition, Prentice Hall, Upper Saddle River, NJ, 2002.

[24] Hovakimyan, Cao, Kharisov, Xargay, and Gregory, "L1 Adaptive Control for Safety-Critical Systems," IEEE Control Systems Magazine, Vol. 31, No. 5, pp. 54-104 October 2011.

[25] J. Shin and T. H. Bond, "Results of an icing test on a NACA 0012 airfoil in the NASA Lewis Icing Research Tunnel," Cleveland, OH, NASA TM 105374, 1992.

[26] Jan Roskam, Flight Dynamics of Rigid and Elastic Airplanes Part One, n.p., USA, 1972.

[27] Jean-Jacques E. Slotine and Weiping Li, Applied Nonlinear Control, PrenticeHall Inc., Englewood Cliffs, NJ, 1991.

[28] Karl J. Astrom and Bjorn Wittenmark, Adaptive Control, $2^{\text {nd }}$ Edition, AddisonWesley Publishing Company Inc., Reading, MA, 1995.

[29] Katsuhiko Ogata, Modern Control Engineering, $5^{\text {th }}$ Edition, Prentice Hall Inc., Upper Saddle River, NJ, 2010.

[30] L. Xin, B. Junqiang, H. Jun, W. Kun, and Z. Yang, "A spongy icing model for aircraft icing," Chinese Journal of Aeronautics, Vol. 27, No. 1 (2014), pp. 40-51. 
[31] Major Larry Crews USAF (Ret.), Interview, 24 March 2014.

[32] M.B. Bragg, T. Hutchison, J. Merret, R. Oltman and D. Pokhariyal, "Effect of ice accretion on aircraft flight dynamics," Proceedings of the $38^{\text {th }}$ AIAA Aerospace Sciences Meeting and Exhibit, Reno, NV, Jan. 2000.

[33] M.B. Bragg, W. R. Perkins, N. B. Sarter, T. Basar, P. G. Voulgaris, H. M. Gurbacki, J. W. Melody, and S.A. McCray, "An Interdisciplinary Approach to Inflight Aircraft Icing Safety," Proceedings of the $36^{\text {th }}$ AIAA Aerospace Sciences Meeting and Exhibit, Reno, NV, Jan. 1998.

[34] M.B. Bragg, "Aircraft aerodynamic effects due to large-droplet ice accretions," Proceedings of the $34^{\text {th }}$ AIAA Aerospace Sciences Meeting and Exhibit, Reno, NV, Jan. 1996.

[35] M.K. Politovich, "Aircraft icing caused by large supercooled drops," Journal of Applied Meteorology vol. 28, no. 9, September 1989, pp. 856-868.

[36] M. V. Cook, Flight Dynamics Principles, $3^{\text {rd }}$ Edition, John Wiley and Sons Inc., New York, NY, 2013.

[37] Michael D. Greenberg, Advanced Engineering Mathematics, $2^{\text {nd }}$ Edition, Prentice Hall Inc., Upper Saddle River, NJ, 1998.

[38] M. Papadakis et al., "Experimental study of supercooled large droplet impingement effects," Wichita, KS, DOT/FAA/AR-03/59, 2003.

[39] National Transportation Safety Board, NTSB Accident Database 8 Synopses [Online], Available: http://www.ntsb.gov/aviationquery/index.aspx 
[40] National Transportation Safety Board, "In-flight icing encounter and loss of control, Simmons Airlines, d.b.a American Eagle Flight 4184, Avions de Transport Regional (ATR)," Roselawn, IN, NTSB/AAR-96/01, 1996.

[41] National Transportation Safety Board, "Crash during takeoff in icing conditions, Canadair, Ltd., CL-600-2A12, N873G," Montrose, CO, NTSB/AAB-06/03, 2006.

[42] National Transportation Safety Board, "Factual Report Aviation," Oak Glen, CA, LAX06FA131, 2006. Available: http://dms.ntsb.gov/aviation/ AccidentReports/cgqjim55jrkt2w45lfew2o451/X04032014120000.pdf

[43] National Transportation Safety Board, "Crash During Approach to Landing Circuit City Stores, Inc. Cessna Citation 560, N500AT," Pueblo, CO, NTSB/AAR07/02, 2007.

[44] N. Hovakimyan and C. Cao, L1 Adaptive Control Theory: Guaranteed Robustness with Fast Adaptation, Series: Advances in Design and Control, SIAM, Philadelphia, PA, 2010

[45] Norman S. Nise, Control Systems Engineering, $6^{\text {th }}$ Edition, John Wiley and Sons Inc., Hoboken, NJ, 2011.

[46] R. K. Jeck, "A history and interpretation of aircraft icing intensity definitions and FAA rules for operating in icing conditions," Atlantic City International Airport, NJ, DOT/FAA/AR-01/91, 2001. 
[47] R. Miller and W. Ribbens, "The effects of icing on the longitudinal dynamics of an icing research aircraft," Proceedings of the $37^{\text {th }}$ AIAA Aerospace Sciences Meeting and Exhibit, Reno, NV, Jan. 1999.

[48] R.J. Shaw, R.G. Sotos, and F.R. Solano, "An expiremental study of airfoil icing characteristics," NASA Lewis Research Center, Cleveland, OH, NASA TM 82790, 1982.

[49] S. G. Cober, G. A. Isaac, and J. W. Strapp, "Characterizations of aircraft icing environments that include supercooled large drops," Journal of Applied Meteorology and Climatology, vol. 40, no.11, pp.1984-2002, 2001.

[50] S. G. Cober and G. A. Isaac, "Characterization of aircraft icing environments with supercooled large drops for application to commercial aircraft certification," Journal of Applied Meteorology and Climatology, vol. 51, no. 2, pp. 265-284, 2012.

[51] S. Jung, M. K. Tiwari, V. Doan and D. Poulikakos, "Mechanism of supercooled droplet freezing on surfaces," Nature Communications, vol. 3, no. 615, pp.1-8, 2012 .

[52] S. Ying and J. Ai, "Detection of aircraft in-flight icing in non-steady atmosphere using artificial neural network," 2010 Third International Symposium on Intelligent Information Technology and Security Informatics (IITSI), Jinggangshan, China, April 2010. 
[53] T. G. Myers, "Extension to the Messinger Model for aircraft icing," American Institute of Aeronautics and Astronautics Journal, vol. 39, no. 2, pp. 211-218, 2001.

[54] T. P. Ratvasky, B. P. Barnhart, and S. Lee, "Current methods modeling and simulating icing effects on aircraft performance, stability, control," Journal of Aircraft, Vol. 47, No. 1 (2010), pp. 201-211.

[55] W. Fuchs, "Forecast of aircraft icing by use of boundary layer model products: first experiences," International Society of Offshore and Polar Engineers, Honolulu, HI, May 2003.

[56] W.J. Baars, R. O. Stearman, and C. E. Tinney, "A review on the impact of icing on aircraft stability and control," Journal of Aeroelasticity and Structural Dynamics vol. 2, no. 1, pp. 35-52, Sep 2010.

[57] W. Olsen, R. Shaw, and J. Newton, "Ice shapes and the resulting drag increase for a NACA 0012 airfoil," NASA TM 83556, Jan. 1984. 